\title{
The orbits of 48 globular clusters in a Milky-Way-Like Barred Galaxy
}

\author{
Christine Allen ${ }^{1}$, Edmundo Moreno ${ }^{1}$ and Bárbara Pichardo ${ }^{1,2}$
}

\begin{abstract}
The effect of a barred potential (such as the one of the Milky Way) on the galactic orbits of forty-eight globular clusters for which absolute proper motions are known is studied. The orbital characteristics are compared with those obtained for the case of an axisymmetric galactic potential. Tidal radii are computed and discussed for both the better known axisymmetric case and that including a bar. The destruction rates due to bulge and disk shocking are calculated and compared in both galactic potentials.
\end{abstract}

Subject headings: galaxy: halo — galaxy: kinematics and dynamics — globular clusters: general

\section{INTRODUCTION}

A considerable number of absolute proper motions for globular clusters have become available in recent times, largely due to the work of Dinescu et al. (1997, 1999a,b, 2000, 2001, 2003). With the new proper motions, full space velocities can now be calculated. Then, using a model for the galactic mass distribution, it is possible to study the galactic orbits of a good fraction -almost a third- of the known globular clusters. Vice-versa, through their orbital motions, globular clusters can serve as kinematical probes of the gravitational field of our Galaxy.

Some of the first efforts in orbit computations for globular clusters are due to the work of Keenan et al. (1973) and Keenan \& Innanen (1975) who numerically integrated orbits for M67, NGC 188, Omega Cen, and NGC 2420 using full space motions in a Schmidt-type potential. With a more realistic galactic potential that included a massive halo, Allen \&

\footnotetext{
${ }^{1}$ Instituto de Astronomía, Universidad Nacional Autónoma de México, Apdo. Postal 70-264, 04510, México, D. F., México.

${ }^{2}$ Institute for Theoretical Physics, University of Zurich, Winterthurerstrasse 190, Zurich 8057, Switzerland.
} 
Martos (1988) and Allen (1990) computed orbits for a total of 15 clusters -all those with then available absolute proper motions. Using an improved version of their axisymmetric potential, Allen \& Santillán (1993) recomputed these orbits and added those of a further six clusters whose absolute proper motions had been determined in the intervening years.

It is only quite recently that vastly improved measures for a significant number of clusters have become available (Dinescu et al. 1997, 1999a,b, 2000, 2001, 2003). These authors use the Yale proper motion survey of the southern sky and measure motions directly with respect to external galaxies, or in difficult cases, to Hipparcos reference stars. Dinescu et al. (1999b) have also used the full space motions (obtained from their proper motions and radial velocities taken from the literature) to compute galactic orbits for 38 clusters in several models for axisymmetric potentials. From the galactic orbits they have derived a number of interesting conclusions.

The importance of using full space motions -instead of only radial velocities- when attempting to derive characteristics of the globular cluster system has been repeatedly emphasized (Webbink 1998; Dinescu et al. 1999b; King 2004). Indeed, using only partial information -radial velocities- makes it necessary to assume some statistical distribution for two components of the space velocity. With the availability of full space motions for about a third of the known clusters, this approach seems less and less justified, especially since in some cases its results may be misleading.

However, since evidence for our Galaxy harboring a bar has become increasingly convincing, it appears necessary to study the influence of this bar on the galactic motions of the clusters as obtained from their full space velocities and employing a galactic potential that includes a bar. These orbits can serve the purpose of calculating tidal shock effects on the cluster. They can also serve as kinematical probes of the inner part of our Galaxy, and so aid in constraining some dynamical parameters of the bar, or at least in establishing whether or not the model potential -especially the bar- is compatible with observed characteristics of the clusters.

In this paper, we analyze the orbits in a galactic potential with a bar of 48 globular clusters with available absolute proper motions. The potential we use is that of Pichardo et al. (2004), which is based on the axisymmetric model of Allen \& Santillán (1991) and includes a moderately strong bar. We compare the orbits with those obtained in the axisymmetric potential, devoting special attention to orbits that reside entirely within the bar region.

The orbits generally do not show secular changes in the total energy, $E$, or in the z-component of the angular momentum, $h$, both computed in the inertial galactic frame. Indeed, with few exceptions, those quantities are on the average conserved within 10 percent. 
However in a few cases, sudden changes in $E$ and $h$ do occur. Except for orbits residing entirely within the bar region, the orbits do not differ considerably from those obtained in the axisymmetric case. This similarity also applies to the tidal-shock destruction rates computed in both galactic potentials.

In Section 2 the galactic potential and the observational data to compute the initial conditions of the clusters' orbits are presented. In Section 3 we show meridional orbits of some clusters, and some effects of the bar are considered. The effect of the bar on the estimated tidal radii is studied in Section 4, and compared with the axisymmetric case. Destruction rates of clusters in both the axisymmetric and non-axisymmetric potentials are studied in Section 5. Section 6 presents our conclusions.

\section{THE GALACTIC POTENTIAL AND THE INITIAL CONDITIONS}

For this study we have employed the axisymmetric galactic potential of Allen \& Santillán (1991), and the barred galactic potential of Pichardo et al. (2004). The axisymmetric potential assembles three components, a bulge and a flattened disk of the form proposed by Miyamoto \& Nagai (1975), and a massive spherical halo extending to a radius of $100 \mathrm{kpc}$. The model satisfies the usual observational constraints such as the galactic rotation curve, the perpendicular force at the solar neighborhood, and the local escape velocity $(\approx 536 \mathrm{~km}$ $\left.\mathrm{s}^{-1}\right)$. The adopted parameters of the model are $R_{0}=8.5 \mathrm{kpc}$, and a circular velocity at the Sun's position $V\left(R_{0}\right)=220 \mathrm{~km} \mathrm{~s}^{-1}$. The total mass is $9 \times 10^{11} M_{\odot}$. The local total mass density is $\rho_{0}=0.15 M_{\odot} \mathrm{pc}^{3}$. The resulting Oort constants are $A=12.95 \mathrm{~km} \mathrm{~s}^{-1}$ $\mathrm{kpc}^{1}$ and $B=-12.93 \mathrm{~km} \mathrm{~s}^{-1} \mathrm{kpc}^{1}$. In this work we have used this model as the underlying axisymmetric potential, but replacing $70 \%$ of the bulge mass with a bar.

The potential of the bar is one of the three constructed in Pichardo et al. (2004). The one we have selected represents the best fit to the most important observational characteristics of the galactic bar. It consists of a superposition of four ellipsoids, each one with a self-similar mass distribution (Schmidt 1956) to simulate the total density profile. The superposition of the ellipsoids is carried out so as to reproduce the apparent "boxy" edge-on brightness profile of the galactic bar. With the selected parameters (chosen from a compilation of recent observations, see references in Pichardo et al. (2004)) the bar included in our model has a major semiaxis of $3.13 \mathrm{kpc}$, axial ratios of 1.7:0.64:0.44, and moves with an angular velocity of $60 \mathrm{~km} \mathrm{~s}^{-1} \mathrm{kpc}^{-1}$. In this way, the bar model closely approximates the Model S of Freudenreich (1998), based on COBE/DIRBE infrared observations of the galactic bar. Throughout this study we will use this so-called "superposition model", which is the most accurate, albeit the most complicated numerically. 
In their study, Pichardo et al. (2004) obtained galactic orbits for three clusters, NGC 5139, NGC 6093, and NGC 6218, selected because their orbits in axisymmetric potentials indicated that they reside mostly within the region of the bar, and so the influence of the bar on their orbits was expected to be large, as indeed it turned out to be. We now extend this study to the complete sample of clusters.

For the initial conditions we take the absolute proper motions provided by Dinescu et al. (1999b, 2000, 2001, 2003), but we adopt for 47 Tuc (NGC 104) and M4 (NGC 6121) the values derived from HST measures (Anderson \& King 2003; Bedin et al. 2003). Other data are taken from the compilation by Harris (1996). We consider the solar motion as $(U, V, W)_{\odot}=$ $(-10,5.2,7.2) \mathrm{km} \mathrm{s}^{-1}$ (Binney \& Merrifield 1998). Once the space velocities are obtained, we integrate the orbits backwards in time for $1.6 \times 10^{10}$ years. For the integration we use the Bulirsch-Stoer algorithm of Press et al. (1992). In the axisymmetric case the relative errors in the total energy were $10^{-14}$ at the end of the integration. In the barred potential, the orbits are computed in the non-inertial reference frame where the bar is at rest. In this case the precision of the calculations can be checked using Jacobi's constant. The relative errors in this quantity turn out to be, typically, $10^{-10}-10^{-11}$.

\section{THE GALACTIC ORBITS}

We have computed orbits for the full sample of 48 clusters. The orbits are computed in the axisymmetric and non-axisymmetric potentials. Figures 1 and 2 show a few meridional

orbits as representative examples. For comparison, for a given cluster we show the meridional orbits in both galactic potentials. Tables 1 and 2 summarize our results for both cases. In Table 1, corresponding to the axisymmetric potential, successive columns contain the name of the cluster, the minimum perigalactic distance reached in the course of the complete orbit, the average perigalactic distance, the maximum apogalactic distance, the average apogalactic distance, the maximum distance from the galactic plane reached throughout the entire orbit, the average maximum distance from the galactic plane, the average orbital eccentricity, the orbital energy per unit mass, the z- component of angular momentum per unit mass, two values for the computed tidal radii (see discussion in Section 4), and the observed limiting radius, given in Harris (1996). Table 2, for the non-axisymmetric potential, is similar; but since neither the orbital energy, $E$, nor the z- component of angular momentum, $h$, (both computed in the inertial galactic frame) are constants of motion, we give in the ninth and tenth columns the minimum and maximum values attained by $h$ in the course of the complete orbit. In order to assess the influence of observational uncertainties on our results, we have listed for each system two additional lines, corresponding to the orbits of maximum and 
minimum energy as discussed at the end of this section.

Many orbits are not noticeably affected by the bar. This is true for all 'outer' clusters, i.e., those with orbits residing entirely outside the bar region. The two left columns of Figure 1 show the meridional orbits of NGC 1904, NCG 2298, NGC 4590 and Pal 12 as examples of this situation. A similar behavior is exhibited by the orbits of NGC 104, NGC 1851, NGC 5024, NGC 5272, NGC 5466, Pal 5, NGC 5904, NGC 6205, NGC 6934, NGC 7006, NGC 7089 and Pal 13.

A completely different situation occurs for orbits residing within, or almost within, the bar region, as was already noted by Pichardo et al. (2004). The orbits show great changes, tending to reach higher values of apogalactic distance and distance to the galactic plane. The two right columns of Figure 1 and the two left columns of Figure 2 show as examples the orbits of NGC 5139, NGC 6171, NGC 6266, NGC 6362, NGC 6254, NGC 6316, NGC 6528, and NGC 6723. A similar behavior is shown by the orbits of NGC 6093, NGC 6121, NGC 6144, NGC 6218, NGC 6304, NGC 6522, NGC 6626, NGC 6712, and NGC 6809. The orbits of NGC 6522, NGC 6752, and NGC 6838 (these last two having their perigalactic points near the boundary of the bar region) reach considerably larger values of apogalactic distance in the presence of the bar than in the axisymmetric case. NGC 6304, NGC 6316, and NGC 6723 have near-resonant orbits in the axisymmetric potential, but get pushed towards greater apogalactic distance by the bar.

Other interesting effects of the bar on the orbits are illustrated in the two right columns of Figure 2. We show the orbits of NGC 7078, NGC 7099, NGC 288, and NGC 6218. In the case of NGC 7078 and NGC 7099, the bar tends to trap the orbit into a near-resonance. The opposite effect can also occur, as seen in the case of NGC 288. Here, the bar pushes the orbit away from a near-resonance. Something similar happens for the orbits of NGC 6553 and NGC 6779. Also shown in Figure 2 is the case of NGC 6218, an orbit in near-resonance in the axisymmetric potential, and fully disordered in the presence of the bar.

Finally, the orbit of Pal 3 may be an escape orbit when run with the available data. An improvement of the observed parameters -distance and proper motion- will most probably yield a normal, bound, orbit.

Plots of the run of energy, $E$, and z-component of angular momentum, $h$, were obtained for all clusters. These quantities are, of course, conserved in the axisymmetric case. In the presence of the bar, the orbits generally do not show secular changes in $E$ or $h$. Indeed, with a few exceptions, these quantities are conserved on the average within better than 10 percent. Figure 3 illustrates this behavior for the cases of NGC 104 and NGC 288. However, in a few cases, abrupt changes in $E, h$ do occur, as shown in Figure 3 for NGC 6809. Here, on top of 
the nearly-periodic changes, a jump occurs, moving the energy and angular momentum to higher values (with increasing time) at the expense of the bar. Note that in the case of NGC 6809, with an extremely low angular momentum orbit, the abrupt changes in the angular momentum actually reverse the sense of revolution of the orbit around the galactic center. Other examples of this behavior are the orbits of NGC 6093, NGC 6316, and NGC 6528.

To take into account the influence observational uncertainties we computed for each cluster two additional orbits in both the axisymmetric potential and the time-varying barred potential. Initial conditions for these orbits were chosen so as to maximize and minimize the energy. In other words, we combined the observational uncertainties in such a way as to obtain two extreme orbits. Errors in the computed orbital parameters resulting from observational uncertainties are expected to be bounded by these extreme orbits. The real uncertainties in our computed results will be much smaller than those shown by the extreme orbits. In Tables 1 and 2 we display two additional lines for each system, corresponding to the minimum and the maximum energy orbits, in both the axisymmetric and the barred potential. As can be seen from a comparison of the three orbits, the errors in the computed parameters are quite moderate, except for NGC 1904, NGC 2298, Pal 3, NGC 4147, and NGC 7006. The parameters most affected by errors are $\left(r_{\min }\right)_{\min },\left\langle r_{\min }\right\rangle$, E and h. Interestingly, Pal 3, the only escape orbit we found, becomes bound in the minimum energy case, whereas NGC 7006 becomes unbound in the maximum energy case. So, within the observational uncertainties, all our computed orbits are bound, as would be expected. Of course, it would be particularly valuable to obtain improved proper motions and distances for the clusters showing the largest uncertainties.

In Section 4 below we discuss the influence of observational uncertainties on the computed tidal radii.

\section{TIDAL RADII}

As a first step in assessing the effects of the bar on the internal dynamics of the clusters, we have computed tidal radii. The correct formula to use for the purpose of comparing the theoretical tidal radii with the observed limiting radii is a matter of some controversy (Brosche et al. 1999, and references therein). We are well aware of the fact that a 'tidal radius' is only a very coarse approximation to the dynamical processes occurring in a cluster. In every cluster we have computed the tidal radius given by King's (1962) formula $r_{K}=$ $\left[M_{c} / M_{g}(3+e)\right]^{1 / 3} r_{\text {min }}$, with $M_{c}$ the cluster's mass, $M_{g}$ an 'effective galactic mass', $e$ the orbital eccentricity, and $r_{\min }$ the galactocentric distance of a perigalactic point in the orbit. As discussed in Appendix A, we also compute the tidal radius of a cluster using an alternative 
approximate formula. This formula is given by Eq. (A2),

$$
r_{*}=\left[\frac{G M_{c}}{\left(\frac{\partial F_{x^{\prime}}}{\partial x^{\prime}}\right)_{\mathbf{r}^{\prime}=0}+\dot{\theta}^{2}+\dot{\varphi}^{2} \sin ^{2} \theta}\right]^{1 / 3} .
$$

In this equation, $F_{x^{\prime}}$ is the component of the galactic acceleration along the line $x^{\prime}$ joining the cluster with the galactic center, and its partial derivative is evaluated at the position of the cluster. The angles $\varphi$ and $\theta$ are angular spherical coordinates of the cluster in an inertial galactic frame. The tidal radius $r_{*}$ is computed at a perigalactic point in the orbit. In a two-body problem, $r_{*}$ coincides with $r_{K}$ given by King's (1962) formula. The main advantage in the computation of $r_{*}$ is that we do not need to make an assumption about an 'effective galactic mass'; an assumption which is unclear if the perigalactic point lies in a region with a strong non-axisymmetric potential, as in the case of a bar.

Figure 4 shows the comparison of the tidal radii $r_{K}$ and $r_{*}$, and their relation with the observed limiting radius $r_{L}$. In Figure 4 (a), (b) we compare $r_{K}$ and $r_{*}$ in the axisymmetric and non-axisymmetric potentials, respectively. Each point corresponds to a cluster, and we are plotting the averages of the tidal radii over the last $10^{9} \mathrm{yr}$ in every cluster's orbit. These frames show that $r_{K}$ and $r_{*}$ give similar values. This conclusion is maintained if we compare $r_{K}$ and $r_{*}$ in a given orbit at any perigalactic point. In Figure 4 (c), (d) we compare $r_{*}$ and $r_{K}$ with the observed limiting radius $r_{L}$. Both frames include the results obtained with the axisymmetric (filled squares) and non-axisymmetric (empty squares) potentials. In general, the values of $r_{*}, r_{K}$ obtained with the non-axisymmetric potential are almost the same as those obtained with the axisymmetric potential (this can also be seen comparing (a) and (b) of Figure 4). We also note that both frames are very similar (because $r_{*}$ and $r_{K}$ are similar), and there is a considerable scatter, mainly in the lower right-hand side in these frames. In order to discuss this scatter, in Figure 5 we compare $r_{*}$ with $r_{L}$, but showing only the results obtained in the axisymmetric potential. To estimate the lower and upper bounds in $r_{*}$, we have computed for each cluster the minimum and maximum energy orbits permitted by the available data. These orbits give the empty squares and empty triangles in Figure 5. We also show in this figure the estimated observational uncertainties in $r_{L}$. Three outliers contribute most of the noise: the points lying in the lower right-hand side of the diagram. As shown in the figure, these points correspond to NGC 6144, Pal 5, and NGC 5466. All three are low-mass, low-concentration clusters. Pal 5 appears to be in the final stages of dissolution (Koch et al. 2004), and there are indications that NGC 5466 is also dissolving (Odenkirchen \& Grebel 2004; Belokurov et al. 2006). The case of NGC 6144 is commented below, in relation with Figure 6 for the non-axisymmetric potential. Thus, in the cases of Pal 5 and 
NGC 5466 the present limiting radii of these clusters do not necessarily correspond to the conditions of the dynamical model for the theoretical tidal radii (which assumes the cluster has undergone a recent episode of tidal pruning). Other clusters lying significantly below the line of coincidence in Figure 5, are NGC 5272, NGC 5139, and NGC 5904, for all three of which tidal tails have been detected (Leon et al. 2000), thus indicating a similar situation to that of Pal 5, if not as extreme. Therefore, agreement between the theoretical tidal radii and the observed limiting radii is not to be expected for these clusters either, as indeed is not observed.

Interestingly, most of the clusters lying significantly above the line of coincidence (NGC 1851, NGC 1904, NGC 2298, NGC 5024, NGC 6205, and NGC 7089) have been proposed as possibly being accreted systems, because they are old clusters with young halo kinematics (Mackey \& Gilmore 2004). This is also the case of NGC 5904 and Pal 12, clusters that lie below the line of coincidence; Dinescu et al. (2000) find that Pal 12 has probably been captured from the Sagittarius dwarf galaxy. A few of the clusters that do not fill their tidal radii (NGC 1851, NGC 1904, and NGC 6205) also exhibit tidal tails (Leon et al. 2000). Taking into account the preceeding considerations, we can say that in general the agreement between the computed and the observed tidal radii of 'normal' clusters is quite good. The outstanding exception is NGC 104, whose observed limiting radius is much smaller than its tidal radius, i.e., for no reason that we can discern, this cluster does not fill its tidal radius.

Figure 6 shows the comparison of $r_{*}$ with $r_{L}$ in the non-axisymmetric potential. The details in this figure are analogous to those in Figure 5; additionally, the points with a circle correspond to clusters with a retrograde galactic orbit, or with an orbit which is both prograde and retrograde, and with a mean perigalactic distance less than $3 \mathrm{kpc}$. Only in the galactic non-axisymmetric potential, which has a rotating bar, the sense of orbital rotation around the galactic center acquires an importance. Some of the clusters with a retrograde galactic orbit and mean perigalactic distance within the bar region, and which do not lie near the line of coincidence in Figure 6, may partly do so because in the computation of a tidal radius in this non-axisymmetric potential, in principle the sense of orbital rotation must be included, which is not the case in $r_{K}$ and $r_{*}$. Keenan \& Innanen (1975) showed that stars within a cluster moving in retrograde orbits are more stable in a tidal field than stars moving in prograde orbits. This effect is not included in the computation of $r_{K}$ and $r_{*}$ either. Figure 6 suggests that clusters moving in retrograde orbits in the galactic non-axisymmetric potential are more stable to the tidal field than clusters moving in prograde orbits; this is because several points corresponding to retrograde orbits lie below the line of coincidence, that is, $r_{L}$ is greater than $r_{*}$ (or $\left.r_{K}\right)$. Examples of this effect are the orbits of NGC 6144 and NGC 6528 (see Figure 6); note that NGC 6528 is the cluster with the smallest computed theoretical tidal radius. 


\section{DESTRUCTION RATES}

We summarize in Sections 5.1 and 5.2 some equations employed to compute destruction rates of globular clusters due to gravitational shocks produced by a galactic bulge and a galactic disk. In Section 5.3 we apply this theory to globular clusters in our Galaxy.

Destruction rates of globular clusters in an axisymmetric model of our Galaxy were obtained in the pioneer work of Aguilar et al. (1988), who considered evaporation due to two-body relaxation, dynamical friction, and gravitational shocks with both the galactic bulge and galactic disk. The effect of a galactic bar on these destruction rates was analyzed by Long et al. (1992). For gravitational shocks, Gnedin \& Ostriker (1997, hereafter G\&O97) considered the effect of the energy change $\langle\Delta E>$ as well as of the second-order term $<(\Delta E)^{2}>$, on a cluster's evolution, introducing in each case adiabatic corrections to the impulse approximation with a power-law form (Gnedin \& Ostriker 1999, hereafter G\&O99), as improvements to the exponential forms given by Spitzer (1987) and Kundic \& Ostriker (1995). Gnedin et al. (1999a) also improved the treatment computing the tidal field due to an extended spherically symmetric mass distribution, as in a spherical galactic bulge.

\subsection{Bulge Shock}

When a globular cluster reaches a perigalactic point with position $\boldsymbol{r}_{p}$ and velocity $\boldsymbol{v}_{p}$ with respect to the galactic inertial frame, the gravitational shock with the bulge, of total mass $M_{b}$, produces a change in binding energy per unit mass given by (taking an average over the cluster of equation (17) in G\&O97)

$$
<(\Delta E)_{b}>=\frac{1}{3}\left(\frac{2 G M_{b}}{\left|\boldsymbol{v}_{p}\right|\left|\boldsymbol{r}_{p}\right|^{2}}\right)^{2} \chi\left(\left|\boldsymbol{r}_{p}\right|\right)<r^{\prime 2} \eta_{1}\left(\beta\left(r^{\prime}\right)\right)>\lambda\left(\left|\boldsymbol{r}_{p}\right|,\left|\boldsymbol{r}_{a}\right|\right) .
$$

The factor $\chi\left(\left|\boldsymbol{r}_{p}\right|\right)$ takes into account the extended mass distribution of the bulge, and is given in equation (18) of G\&O97 (see also equation (17) of Gnedin et al. (1999a)). In Appendix B we show that the expression they give for this factor can be simplified, and needs the computation of only two integrals over the mass distribution of the bulge. Thus, using the results obtained in Appendix B we have

$$
\chi\left(\left|\boldsymbol{r}_{p}\right|\right)=\frac{1}{2}\left[\Im_{1}^{2}\left(\left|\boldsymbol{r}_{p}\right|\right)+\left(1-\frac{4 \pi\left|\boldsymbol{r}_{p}\right|^{3}}{M_{b}} \Im_{2}\left(\left|\boldsymbol{r}_{p}\right|\right)\right)^{2}\right]
$$

with 


$$
\begin{aligned}
& \Im_{1}\left(\left|\boldsymbol{r}_{p}\right|\right)=\int_{1}^{\infty} \frac{\mu_{b}\left(u\left|\boldsymbol{r}_{p}\right|\right) d u}{u^{2}\left(u^{2}-1\right)^{1 / 2}}, \\
& \Im_{2}\left(\left|\boldsymbol{r}_{p}\right|\right)=\int_{1}^{\infty} \frac{\rho_{b}\left(u\left|\boldsymbol{r}_{p}\right|\right) u^{3} d u}{\left(u^{2}-1\right)^{1 / 2}} .
\end{aligned}
$$

Here $\mu_{b}(r)=M_{b}(r) / M_{b}$, with $M_{b}(r)$ the mass of the bulge within a sphere of galactocentric radius $r, M_{b}$ is the total mass of the bulge, and $\rho_{b}(r)$ is the density of the spherically symmetric bulge as a function of galactocentric distance $r$.

The factor $<r^{\prime 2} \eta_{1}\left(\beta\left(r^{\prime}\right)\right)>$ in Eq. (2) is an average over the cluster; $r^{\prime}$ is distance from the center of the cluster, $\beta\left(r^{\prime}\right)$ is the adiabatic parameter $\beta\left(r^{\prime}\right)=2\left|\boldsymbol{r}_{p}\right| \omega\left(r^{\prime}\right) /\left|\boldsymbol{v}_{p}\right|$, with $\omega\left(r^{\prime}\right)$ angular velocity of stars inside the cluster. Considering circular motions as representative, then $\omega\left(r^{\prime}\right)=\left[G M_{c}\left(r^{\prime}\right) / r^{\prime 3}\right]^{1 / 2}$, with $M_{c}\left(r^{\prime}\right)$ the mass of the cluster within a sphere of radius $r^{\prime}$. The function $\eta_{1}(\beta)\left(A_{b 1}\right.$ in the notation of G\&O97) is the adiabatic correction of the form given by G\&O99: $\eta_{1}(\beta)=\left(1+\frac{1}{4} \beta^{2}\right)^{-\gamma_{1}}\left(\beta=2 x\right.$ in their notation). The exponent $\gamma_{1}$ is given in Table 2 of G\&O99, and we extended its values as follows: $\gamma_{1}=2.5$ if $\tau \equiv\left|\boldsymbol{r}_{p}\right| /\left|\boldsymbol{v}_{p}\right| \leq t_{d y n, h}$, $\gamma_{1}=2$ if $t_{d y n, h}<\tau<4 t_{d y n, h}, \gamma_{1}=1.5$ if $\tau \geq 4 t_{d y n, h}$, with $t_{d y n, h}=\left(\pi^{2} r_{h}^{2} / 2 G M_{c}\right)^{1 / 2}$ the half-mass dynamical time, where $r_{h}^{\prime}$ is the half-mass radius and $M_{c}$ the mass of the cluster. The average $<r^{\prime 2} \eta_{1}\left(\beta\left(r^{\prime}\right)\right)>$ is taken as

$$
<r^{2} \eta_{1}\left(\beta\left(r^{\prime}\right)\right)>=\frac{4 \pi}{M_{c}} \int_{0}^{r_{t}^{\prime}} \rho_{c}\left(r^{\prime \prime}\right) \eta_{1}\left(\beta\left(r^{\prime \prime}\right)\right) r^{\prime \prime 4} d r^{\prime \prime}
$$

with $r_{t}^{\prime}$ the tidal radius of the cluster and $\rho_{c}\left(r^{\prime}\right)$ its spatial density, obtained with a King (1966) model.

The factor $\lambda\left(\left|\boldsymbol{r}_{p}\right|,\left|\boldsymbol{r}_{a}\right|\right)$ in Eq. (2), a function of the perigalactic, $\left|\boldsymbol{r}_{p}\right|$, and apogalactic, $\left|\boldsymbol{r}_{a}\right|$, distances, is an approximation for the effect of the time variation of the galactic tidal force along the orbit of the cluster. According to Aguilar et al. (1988) this factor can be taken as

$$
\lambda\left(\left|\boldsymbol{r}_{p}\right|,\left|\boldsymbol{r}_{a}\right|\right)=\left[1-\frac{M_{b}\left(\left|\boldsymbol{r}_{a}\right|\right)}{M_{b}\left(\left|\boldsymbol{r}_{p}\right|\right)}\left(\frac{\left|\boldsymbol{r}_{p}\right|}{\left|\boldsymbol{r}_{a}\right|}\right)^{3}\right]^{2} .
$$

The second-order term $<(\Delta E)_{b}^{2}>$ in the gravitational shock with the bulge is given by equation (19) of Gnedin et al. (1999a), modified by the factors $\chi\left(\left|\boldsymbol{r}_{p}\right|\right), \lambda\left(\left|\boldsymbol{r}_{p}\right|,\left|\boldsymbol{r}_{a}\right|\right)$ and the appropriate adiabatic correction factor $\eta_{2}\left(\beta\left(r^{\prime}\right)\right)$. Thus, an average over the cluster gives 


$$
<(\Delta E)_{b}^{2}>=\frac{2}{9}\left(\frac{2 G M_{b}}{\left|\boldsymbol{v}_{p}\right|\left|\boldsymbol{r}_{p}\right|^{2}}\right)^{2} \chi\left(\left|\boldsymbol{r}_{p}\right|\right)<r^{\prime 2} v^{\prime 2} \eta_{2}\left(\beta\left(r^{\prime}\right)\right)\left(1+\chi_{r^{\prime}, v^{\prime}}\right)>\lambda\left(\left|\boldsymbol{r}_{p}\right|,\left|\boldsymbol{r}_{a}\right|\right)
$$

and we have approximated the rms velocity $v^{\prime}$ within the cluster with that given by circular motion; thus $r^{\prime 2} v^{\prime 2}=\omega^{2}\left(r^{\prime}\right) r^{\prime 4}=G M_{c}\left(r^{\prime}\right) r^{\prime}$ (see equation (22) of G\&O97). The adiabatic correction is $\eta_{2}(\beta)=\left(1+\frac{1}{4} \beta^{2}\right)^{-\gamma_{2}}$, with $\gamma_{2}$ given in Table 2 of G\&O99 and extended as follows: $\gamma_{2}=3$ if $\tau \equiv\left|\boldsymbol{r}_{p}\right| /\left|\boldsymbol{v}_{p}\right| \leq t_{d y n, h}, \gamma_{2}=2.25$ if $t_{d y n, h}<\tau<4 t_{d y n, h}, \gamma_{2}=1.75$ if $\tau \geq 4 t_{d y n, h}$. The position-velocity correlation function $\chi_{r^{\prime}, v^{\prime}}$ is given by G\&O99, and the average $<r^{\prime 2} v^{\prime 2} \eta_{2}\left(\beta\left(r^{\prime}\right)\right)\left(1+\chi_{r^{\prime}, v^{\prime}}\right)>$ is

$$
<r^{\prime 2} v^{\prime 2} \eta_{2}\left(\beta\left(r^{\prime}\right)\right)\left(1+\chi_{r^{\prime}, v^{\prime}}\right)>=\frac{4 \pi G}{M_{c}} \int_{0}^{r_{t}^{\prime}} \rho_{c}\left(r^{\prime \prime}\right) M_{c}\left(r^{\prime \prime}\right) \eta_{2}\left(\beta\left(r^{\prime \prime}\right)\right)\left(1+\chi_{r^{\prime}, v^{\prime}}\left(r^{\prime \prime}\right)\right) r^{\prime \prime 3} d r^{\prime \prime}
$$

Following G\&O97, bulge shock timescales are defined as

$$
\begin{aligned}
& t_{\text {bulge }, 1}=\left(\frac{-E_{c}}{<(\Delta E)_{b}>}\right) P_{o r b}, \\
& t_{b u l g e, 2}=\left(\frac{E_{c}^{2}}{<(\Delta E)_{b}^{2}>}\right) P_{o r b},
\end{aligned}
$$

with $E_{c} \simeq-0.2 G M_{c} / r_{h}^{\prime}$ the mean binding energy per unit mass of the cluster and $P_{\text {orb }}$ its orbital period in the galactic radial direction. The total destruction rate due to gravitational shocks with the bulge is

$$
\frac{1}{t_{b u l g e}}=\frac{1}{t_{b u l g e, 1}}+\frac{1}{t_{b u l g e, 2}}
$$

\subsection{Disk Shock}

For a gravitational shock with a galactic disk, the corresponding expressions of $<(\Delta E)_{d}>$ and $<(\Delta E)_{d}^{2}>$ in a cluster are obtained with averages of equations (1) and (2) in Gnedin et al. (1999b):

$$
<(\Delta E)_{d}>=\frac{2 g_{m}^{2}}{3 v_{z}^{2}}<r^{\prime 2} \eta_{1}\left(\beta\left(r^{\prime}\right)\right)>
$$




$$
<(\Delta E)_{d}^{2}>=\frac{4 g_{m}^{2}}{9 v_{z}^{2}}<r^{\prime 2} v^{\prime 2} \eta_{2}\left(\beta\left(r^{\prime}\right)\right)\left(1+\chi_{r^{\prime}, v^{\prime}}\right)>
$$

with $\left|g_{m}\right|$ the maximum acceleration produced by the disk in the z-direction, perpendicular to its plane, on the perpendicular line at the position where the galactic orbit of the cluster passes through the disk; $\left|v_{z}\right|$ is the z-velocity of the cluster at this point. The adiabatic functions $\eta_{1}(\beta), \eta_{2}(\beta)$ are those employed in the gravitational shock with a bulge, but now $\beta\left(r^{\prime}\right)=2\left|z_{m}\right| \omega\left(r^{\prime}\right) /\left|v_{z}\right|$, with $\left|z_{m}\right|$ the height above the plane of the disk where $\left|g_{m}\right|$ is reached. As before, $\omega\left(r^{\prime}\right)$ is angular velocity of circular motions within the cluster. Also, the parameter $\tau$, whose value gives the corresponding values of the exponents $\gamma_{1}, \gamma_{2}$ in the adiabatic corrections, is $\tau=\left|z_{m}\right| /\left|v_{z}\right|$. The averages in Eqs. (13), (14) are obtained with Eqs. (6), (9).

If the orbit of the cluster has $n$ crossings (not necessarily two) with the disk during the radial orbital period $P_{\text {orb }}$, disk shock timescales and total destruction rate due to gravitational shocks with the disk are defined as

$$
\begin{gathered}
t_{d i s k, 1}=\left(\frac{-E_{c}}{<(\Delta E)_{d}>}\right) \frac{P_{\text {orb }}}{n}, \\
t_{d i s k, 2}=\left(\frac{E_{c}^{2}}{<(\Delta E)_{d}^{2}>}\right) \frac{P_{o r b}}{n}, \\
\frac{1}{t_{d i s k}}=\frac{1}{t_{d i s k, 1}}+\frac{1}{t_{d i s k, 2}} .
\end{gathered}
$$

\subsection{Destruction rates of globular clusters in our Galaxy}

The formulism given in the two previous sections has been applied to globular clusters in our Galaxy, considering the axisymmetric and non-axisymmetric galactic potentials. In the non-axisymmetric galactic potential (Pichardo et al. 2004) the original spherical bulge in the axisymmetric potential is left with a small fraction $(30 \%)$ of its mass, the rest (70 $\%$ goes into the galactic bar. In the Cartesian axes defined by the principal axes of the bar, the density of the bar at a point $(x, y, z)$ is approximately the density given by Model S of Freudenreich (1998): $\rho_{\text {Bar }}(x, y, z) \propto \operatorname{sech}^{2}\left(R_{S}(x, y, z)\right)$ (see Pichardo et al. (2004)). In the upper frame of Figure 7 we show the density of the bar and the spherical bulge, as functions of galactocentric distance and scaled by the central density of the bulge in the axisymmetric 
galactic potential. The dotted line gives the density of this bulge in the axisymmetric galactic potential; the short-dashed line shows the density of the spherical bulge and the long-dashed line gives the density of the galactic bar along its major axis. The continuous line gives the total density of these two components. Notice that the bar now gives a moderate steepness in the run of density, as compared with the original bulge. In our computations in the non-axisymmetric potential, we take as the 'bulge' the galactic bar plus the spherical bulge; hence this bulge is not spherically symmetric. The computation of destruction rates due to bulge and disk shocking is easily done in the axisymmetric galactic potential. However, in the non-axisymmetric galactic potential the computation of $\chi\left(\left|\boldsymbol{r}_{p}\right|\right)$ and $\lambda\left(\left|\boldsymbol{r}_{p}\right|,\left|\boldsymbol{r}_{a}\right|\right)$ in Eqs. (3) and (7) is not immediate, as in these factors the bulge is supposed to be spherically symmetric. Thus, an approximation must be done to compute these factors. Long et al. (1992), who used a bar to evaluate its effect on the destruction rates of globular clusters, must also use some approximation to compute their factor $\chi$.

Our approximation to compute $\chi\left(\left|\boldsymbol{r}_{p}\right|\right)$ and $\lambda\left(\left|\boldsymbol{r}_{p}\right|,\left|\boldsymbol{r}_{a}\right|\right)$ in the non-axisymmetric galactic potential, involves representing the 'bulge' as spherical and transforming the galactic bar to an 'equivalent' spherical bulge. The density of the bar, in terms of Cartesian coordinates along its principal axes, $\rho_{\text {Bar }}(x, y, z) \propto \operatorname{sech}^{2}\left(R_{S}(x, y, z)\right)$, is associated to the galactocentric distance $r=\frac{1}{3}\left(a_{x}+a_{y}+a_{z}\right) R_{S}(x, y, z)$, with $a_{x}, a_{y}, a_{z}$ the scale lengths of the bar (Freudenreich 1998). In the lower frame of Figure 7 we show as a continuous line the ratio $\mu_{b}(r)=M_{b}(r) / M_{b A S}$, with $M_{b}(r)$ the mass of the 'equivalent' total bulge (that is, the spherical bulge plus the 'equivalent' spherical bulge associated with the galactic bar) within a sphere of galactocentric radius $r ; M_{b A S}$ is the total mass of the bulge in the axisymmetric galactic potential. The dotted line in this frame corresponds to the bulge in the Allen \& Santillán (1991) galactic potential. Notice that even with this contracted-bulge approximation, the 'equivalent' total bulge is not the same as the bulge in the axisymmetric potential. We stress the fact that the above approximation is used only to compute $\chi\left(\left|\boldsymbol{r}_{p}\right|\right)$ and $\lambda\left(\left|\boldsymbol{r}_{p}\right|,\left|\boldsymbol{r}_{a}\right|\right)$; but a cluster's galactic orbit is strictly computed in the non-axisymmetric galactic potential. Then $\left|\boldsymbol{r}_{p}\right|,\left|\boldsymbol{v}_{p}\right|$, and $\left|\boldsymbol{r}_{a}\right|$ employed in Section 5.1 are known for any orbital period. In any case, the factor $\lambda\left(\left|\boldsymbol{r}_{p}\right|,\left|\boldsymbol{r}_{a}\right|\right)$ is not at all well determined (Aguilar et al. 1988). A better treatment to compute the effect of the bar, without the above approximation, is under way.

In Table 3 we present the destruction rates obtained in our computations. The first column shows the name of the cluster. The second column gives the mass of the cluster, computed with a mass-to-light ratio of 2. Absolute visual magnitudes are taken from Harris (1996). The only exception is NGC 5139 (Omega Cen), whose mass is taken from Poveda \& Allen (1975). The central concentration and the half-mass radius are given in columns three and four, and are taken from Harris (1996). The remaining columns show the rounded destruction rates due to the bulge and disk (see Eqs. (12) and (17). These destruction 
rates are the averages over the last $10^{9} \mathrm{yr}$ in a cluster's orbit, taking the corresponding perigalactic points and crossings with the galactic disk; this time interval is extended in some cases (NGC 1851, NGC 4147, NGC 4590, NGC 5024, NGC 5466, NGC 5904, NGC 6205, NGC 6934, NGC 7006, NGC 7089, Pal 12, and Pal 13) to allow for a representative number of perigalactic points and crossings with the disk. For a given cluster, the first line in Table 3 shows the destruction rates computed in the axisymmetric potential; the second line gives the corresponding values in the non-axisymmetric potential.

In Figures 8, 9, and 10 we compare the destruction rates due to the bulge, the disk, and bulge and disk, respectively, in the axisymmetric and non-axisymmetric potentials. In these figures, the empty squares, empty triangles, and filled squares show the values obtained with the extreme minimum and maximum energy orbits, and the 'central' orbit, respectively. In Figure 8, some clusters (NGC 1904, NGC 2298, NGC 4147, NGC 5897, NGC 6093, NGC 6316, NGC 6712, NGC 6934, NGC 7006) have large variations of their destruction rates, as given by the extreme orbits. For the rest of the sample the mean deviation from the value given by the 'central' orbit is around 0.9 dex and 0.78 dex in the axisymmetric and non-axisymmetric potentials, respectively. The points (shown as filled squares) with largest deviation from the line of coincidence correspond to clusters with $<r_{\min }><3 \mathrm{kpc}, r_{\min }$ being the perigalactic distance; for these clusters the effect of the bar is important. In Figure 9, the mean deviation of destruction rates from that of the 'central' orbit is 0.4 dex in both potentials, omitting the clusters listed above for which the variation is large. In this case, there is no important deviation from the line of coincidence, even for clusters with perigalactic points inside the bar region. The combined effects of bulge and disk shown in Figure 10, give a corresponding mean deviation of destruction rates of 0.6 dex and 0.57 dex in the axisymmetric and non-axisymmetric potentials, respectively. This figure shows that most clusters have similar total (bulge+disk) destruction rates in both potentials. Figure 11 shows some detail of Figure 10. Here we plot only the the points corresponding to the 'central' orbits, adding some NGC and Pal numbers, and showing with marked squares the clusters with $<r_{\min }><3 \mathrm{kpc}$. In NGC 362, NGC 5897, NGC 6121, and NGC 6584, the total destruction rate is sensibly smaller for the non-axisymmetric potential; considering only the effects of tidal shocks with the bulge and disk, in the barred potential five clusters are expected to be destroyed during the next Hubble time (taken as $10^{10} \mathrm{yr}$ ): Pal 5, NGC 6144, NGC 6362, NGC 6522, and NGC 6528. Considering the interval defined by the extreme minimum and maximum energy orbits, NGC 5897, NGC 6121, and NGC 6584 could also be destroyed during the next Hubble time. In the axisymmetric potential the six clusters expected to be destroyed by the tidal shocks are Pal 5, NGC 5897, NGC 6121, NGC 6144, NGC 6522, and NGC 6528. Considering also the extreme orbits, NGC 6093, NGC 6316, and NGC 6712 could be added to this list. A similar result is obtained by Dinescu et al. (1999b), 
who find that, in their axisymmetric potential, five clusters are destroyed in a Hubble time under the combined effect of the tidal shocks with the bulge and disk (see the bottom left panel in their figure 8). The total destruction rate will increase if we include the evaporation by two-body relaxation; the destruction rates due to this effect are given by G\&O97 in their Table 3 (using the value 3 for the mass-to-light ratio).

In Figure 12 we plot only the values obtained with the 'central' orbits. We show how the destruction rates due to the bulge depend on the mean perigalactic distance, in both the axisymmetric (frame (a)) and non-axisymmetric (frame (c)) potentials. Clusters with perigalactic points close to the Galactic center and large orbital eccentricities (empty squares) have, in general, greater bulge destruction rates. In these frames, points corresponding to clusters with a mass less than $10^{5} M_{\odot}$ are shown as circles. Frames (b) and (d) of Figure 12 give the comparison of the destruction rates due to the bulge and disk, in the axisymmetric and non-axisymmetric potentials, respectively. These frames show that bulge shocking dominates in the bar region, as found by Aguilar et al. (1988).

Figures 8 to 12 show that the destruction rates are very similar in both the axisymmetric and non-axisymmetric Galactic potentials. A similar result was obtained by Long et al. (1992). However, our destruction rates are sensibly smaller than those obtained by G\&O97, who find a much larger destruction rate due to the Galactic bulge and disk. A similar conclusion was reached by Dinescu et al. (1999b) when comparing their results with those of G\&O97, employing an axisymmetric potential. Our approach to compute the Galactic orbits of globular clusters is the same as that considered by Dinescu et al. (1999b), that is, the use of the available data on absolute proper motions and radial velocities. On the other hand, G\&O97 consider a statistical procedure to compute the orbits. In their study, Dinescu et al. (1999b) have computed the destruction rates due to tidal shocks at the half-mass radius, while we have taken an average over the whole cluster, including the adiabatic corrections of G\&O99. However, this improvement in the computation of destruction rates cannot account

for the difference with G\&O97 results. Thus, it would be of great interest to use the rigorous Fokker-Planck approach of G\&O97 along with full space motion data, and galactic orbits, in order to achieve a better estimate of the destruction rates.

\section{CONCLUSIONS}

We have obtained orbits for 48 globular clusters in both a barred and an axisymmetric galactic potential. The orbits of outer clusters (those with pericentric distances greater than about $4 \mathrm{kpc}$ ) are largely unaffected by the bar. The largest changes were found to occur for 'inner' clusters, i.e., those whose orbits reside mostly within the bar region. The main changes 
that the bar causes in the orbits are larger vertical and radial excursions. In general the bar causes no net global changes in the energy or the z-component of the angular momentum, computed in an inertial frame. However, there are cases where jumps in these quantities do occur, even causing a temporary reversal of the sense of rotation of the orbit. Tidal radii have been computed with a new expression, and with a numerical evaluation of the relevant quantities along the orbit. No noticeable changes due to the bar were found for most of the clusters. When changes do occur, they generally make the computed tidal radii somewhat larger in the presence of a bar. We find that the destruction rates due to shocks with the galactic bulge and disk are not strongly affected if we consider a barred galactic potential; in some cases (NGC 362, NGC 5897, NGC 6121, and NGC 6584) the total destruction rate due to both effects can even decrease when using the barred potential. We thus concur with the result of Long et al. (1992), that the similarity of destruction rates in axisymmetric and barred potentials does not rule out the existence of a galactic bar. Although we have included adiabatic corrections in the computation of tidal-shock destruction rates, these rates are smaller than those obtained by G\&O97. Thus, to clarify this difference, the absolute proper motion data should be used with the G\&O97 approach.

\section{A. Alternative Formulation for Estimating the Tidal Radius}

For the motion of a globular cluster of mass $M_{c}$ in a galactic force field, on an orbit of eccentricity $e$, an approximation to the tidal radius of the cluster evaluated at a perigalactic point with galactocentric distance $r_{m i n}$, is given by King's (1962) formula $r_{K}=\left[M_{c} / M_{g}(3+\right.$ e) $]^{1 / 3} r_{\text {min }}$. This formula needs an approximate value of the 'effective' galactic mass $M_{g}$, which can be taken as the point mass that produces the actual force felt by the cluster at the perigalactic point (Allen \& Martos 1988). An alternative approximate formula for the tidal radius of a cluster in a general galactic field can be obtained, requiring only that at certain position on the line joining the cluster and the galactic center, a test particle with velocity $\boldsymbol{v}^{\prime}=0$, measured in a non-inertial reference frame at the center of the cluster, has an acceleration $\boldsymbol{a}^{\prime}$ in this frame with no component along this line.

Take a galactic inertial frame with its origin at the galactic center; Cartesian axes in this frame are $x, y, z$, the $z$ - axis being perpendicular to the galactic plane. A cluster moving in the galactic field has angular spherical coordinates $(\varphi, \theta)$ measured in the inertial frame. The primed non-inertial frame at the cluster has its Cartesian $x^{\prime}$ - axis pointing always toward the galactic center, and the $y^{\prime}$ - axis pointing in the opposite direction of the unit vector in the azimuthal direction, $\boldsymbol{e}_{\varphi}$. The angular velocity of the primed frame with respect to the inertial frame is $\boldsymbol{\Omega}=\dot{\varphi} \boldsymbol{k}+\dot{\theta} \boldsymbol{e}_{\varphi}=-\dot{\varphi} \cos \theta \boldsymbol{i}^{\prime}-\dot{\theta} \boldsymbol{j}^{\prime}+\dot{\varphi} \sin \theta \boldsymbol{k}^{\prime}$; with $\boldsymbol{k}, \boldsymbol{i}^{\prime}, \boldsymbol{j}^{\prime}, \boldsymbol{k}^{\prime}$ the 
unit vectors on the $z, x^{\prime}, y^{\prime}, z^{\prime}$ - axes, respectively. The position of the cluster in the inertial frame is $\boldsymbol{r}_{c}$ and any point in space has a position $\boldsymbol{r}, \boldsymbol{r}^{\prime}$ in the inertial and primed frames, respectively. The acceleration $\boldsymbol{a}^{\prime}$ of a test particle at $\boldsymbol{r}^{\prime}$ is given by (see e.g., Symon (1971)) $\boldsymbol{a}^{\prime}=\boldsymbol{a}-\boldsymbol{a}_{0}-2 \boldsymbol{\Omega} \times \boldsymbol{v}^{\prime}-\boldsymbol{\Omega} \times\left(\boldsymbol{\Omega} \times \boldsymbol{r}^{\prime}\right)-\left(\frac{d}{d t} \boldsymbol{\Omega}\right) \times \boldsymbol{r}^{\prime}$, with $\boldsymbol{a}$ the acceleration at $\boldsymbol{r}^{\prime}$ measured in the inertial frame, and $\boldsymbol{a}_{0}$ the acceleration of the primed frame with respect to the inertial frame. These accelerations have the expressions $\boldsymbol{a}=-G M_{c} \boldsymbol{r}^{\prime} /\left|\boldsymbol{r}^{\prime}\right|^{3}-(\nabla \Phi)_{\mathbf{r}}, \boldsymbol{a}_{0}=-(\nabla \Phi)_{\mathbf{r}_{\mathbf{c}}} ; \Phi$ is the galactic potential per unit mass and the galactic acceleration $-\nabla \Phi$ appears evaluated at the position $\boldsymbol{r}$ of the test particle and at the position $\boldsymbol{r}_{c}$ of the cluster. We are assuming that $\boldsymbol{r}^{\prime}$ lies outside the spherical mass distribution of the cluster. Now we take $\boldsymbol{r}^{\prime}$ on the positive $x^{\prime}$ - axis and find the instantaneous position $x^{\prime}$ at which $\ddot{x}^{\prime}=0$ with $\boldsymbol{v}^{\prime}=0$. The term $\left(\frac{d}{d t} \boldsymbol{\Omega}\right) \times \boldsymbol{r}^{\prime}$ has no $x^{\prime}$ - component, and with $\boldsymbol{\Omega}$ given above the condition satisfied by $x^{\prime}$ is

$$
-\frac{G M_{c}}{x^{\prime 2}}-\boldsymbol{i}^{\prime} \cdot\left[(\nabla \Phi)_{\mathbf{r}}-(\nabla \Phi)_{\mathbf{r}_{\mathbf{c}}}\right]+x^{\prime}\left(\dot{\theta}^{2}+\dot{\varphi}^{2} \sin ^{2} \theta\right)=0
$$

We call $F_{x^{\prime}}=-\boldsymbol{i}^{\prime} \cdot \nabla \Phi$, the $x^{\prime}$ - component of the galactic acceleration along the $x^{\prime}$ - axis. Then $-\boldsymbol{i}^{\prime} \cdot\left[(\nabla \Phi)_{\mathbf{r}}-(\nabla \Phi)_{\mathbf{r}_{\mathbf{c}}}\right] \simeq x^{\prime}\left(\partial F_{x^{\prime}} / \partial x^{\prime}\right)_{\mathbf{r}^{\prime}=0}$ and the required solution of Eq. (A1) is approximately

$$
r_{*} \equiv x^{\prime}=\left[\frac{G M_{c}}{\left(\frac{\partial F_{x^{\prime}}}{\partial x^{\prime}}\right)_{\mathbf{r}^{\prime}=0}+\dot{\theta}^{2}+\dot{\varphi}^{2} \sin ^{2} \theta}\right]^{1 / 3} .
$$

Eq. (A2), which has the form of equation (7) in King's (1962) analysis, can also be obtained if we start with Cartesian axes $x^{\prime}, y^{\prime}, z^{\prime}$ as follows: take the $x^{\prime}$ - axis as before but now the $z^{\prime}$ - axis points in the direction $\boldsymbol{k}^{\prime}=\left(\boldsymbol{r}_{c} \times \boldsymbol{v}_{c}\right) /\left|\boldsymbol{r}_{c} \times \boldsymbol{v}_{c}\right|$, with $\boldsymbol{r}_{c}, \boldsymbol{v}_{c}$ the instantaneous position and velocity of the cluster in the inertial frame. The instantaneous angular velocity of the new primed frame is $\Omega=\boldsymbol{k}^{\prime}\left|\boldsymbol{v}_{c t}\right| /\left|\boldsymbol{r}_{c}\right|$, with $\boldsymbol{v}_{c t}=\left|\boldsymbol{r}_{c}\right| \dot{\varphi} \sin \theta \boldsymbol{e}_{\varphi}+\left|\boldsymbol{r}_{c}\right| \dot{\theta} \boldsymbol{e}_{\theta}$ the tangential velocity of the cluster. Then, with the steps followed above, we arrive again at Eq. (A2).

We can apply Eq. (A2) at any point of the galactic orbit of the cluster, and in particular at a perigalactic point the resulting tidal radius $r_{*}$ can be compared with that obtained using King's (1962) formula, $r_{K}$. 


\section{B. Simplification of the $\chi\left(\left|\boldsymbol{r}_{p}\right|\right)$ Factor}

The analysis made by Spitzer (1958) (see also Spitzer (1987)) for the change of velocity of a particle due to the tidal force arising in the gravitational interaction of two concentrated masses (with the particle bounded to one of these masses), can be easily extended to obtain the change of velocity of a star in a cluster passing by a bulge with a spherically symmetric mass density $\rho_{b}(r)$, with $r$ galactocentric distance. The cluster reaches a pericenter position $\boldsymbol{r}_{p}$ with a velocity $\boldsymbol{v}_{p}$ with respect to a galactic inertial frame. In the impulse approximation the cluster is supposed to move on the line passing at $\boldsymbol{r}_{p}$ with constant velocity $\boldsymbol{v}_{p}$. An inertial Cartesian frame is defined with its center at the cluster; the $x$ - axis is parallel to $\boldsymbol{r}_{p}$, pointing in the $-\boldsymbol{r}_{p}$ direction, and the $y$ - axis points along the line of motion. A star in the cluster has coordinates $x, y, z$. The total changes of the star's velocity components in this inertial frame are

$$
\begin{gathered}
\Delta v_{x}=2 G M_{b} x \int_{0}^{\infty} \frac{\mu_{b}(\xi(t))}{\xi^{3}(t)}\left[2-\frac{3\left|\boldsymbol{v}_{p}\right|^{2} t^{2}}{\xi^{2}(t)}\right] d t+8 \pi G x \int_{0}^{\infty} \rho_{b}(\xi(t))\left[\frac{\left|\boldsymbol{v}_{p}\right|^{2} t^{2}}{\xi^{2}(t)}-1\right] d t, \\
\Delta v_{y}=2 G M_{b} y \int_{0}^{\infty} \frac{\mu_{b}(\xi(t))}{\xi^{3}(t)}\left[\frac{3\left|\boldsymbol{v}_{p}\right|^{2} t^{2}}{\xi^{2}(t)}-1\right] d t-8 \pi G y \int_{0}^{\infty} \rho_{b}(\xi(t)) \frac{\left|\boldsymbol{v}_{p}\right|^{2} t^{2}}{\xi^{2}(t)} d t \\
\Delta v_{z}=-2 G M_{b} z \int_{0}^{\infty} \frac{\mu_{b}(\xi(t))}{\xi^{3}(t)} d t
\end{gathered}
$$

with $\mu_{b}(r)=M_{b}(r) / M_{b}, M_{b}(r)$ is the mass of the bulge within a sphere of galactocentric radius $r, M_{b}$ is the total mass of the bulge, and $\xi(t)=\left(\left|\boldsymbol{r}_{p}\right|^{2}+\left|\boldsymbol{v}_{p}\right|^{2} t^{2}\right)^{1 / 2}$. In the case considered by Spitzer (1958) we have $\mu_{b}(r)=1, \rho_{b}(r)=0$ and Eqs. (B1), (B2), (B3) give his equation (8) (a minus sign is missing in his expression of $\Delta v_{z}$; correct in equation (5.35) of Spitzer (1987)).

Now we change the integrations to the variable $\xi$, and a second change to the variable $u \equiv \xi /\left|\boldsymbol{r}_{p}\right|$. Also, we use $d \mu_{b} / d r=4 \pi r^{2} \rho_{b}(r) / M_{b}$, and following Gnedin et al. (1999a) define $\mu_{b}(r) \equiv r\left(d \mu_{b} / d r\right)=d \mu_{b} / d \ln r$. Thus, Eq. (B2) can be written as

$$
\begin{aligned}
\Delta v_{y}= & \frac{2 G M_{b} y}{\left|\boldsymbol{v}_{p}\right|\left|\boldsymbol{r}_{p}\right|^{2}}\left\{\int_{1}^{\infty} \frac{\mu_{b}\left(u\left|\boldsymbol{r}_{p}\right|\right)}{u^{2}}\left(2-\frac{3}{u^{2}}\right) \frac{d u}{\left(u^{2}-1\right)^{1 / 2}}+\right. \\
& \left.+\int_{1}^{\infty} \frac{\mu_{b}\left(u\left|\boldsymbol{r}_{p}\right|\right)}{u^{2}}\left(\frac{1}{u^{2}}-1\right) \frac{d u}{\left(u^{2}-1\right)^{1 / 2}}\right\}
\end{aligned}
$$


Again, following Gnedin et al. (1999a) we define

$$
\begin{aligned}
& I_{0}\left(\left|\boldsymbol{r}_{p}\right|\right)=\int_{1}^{\infty} \mu_{b}\left(u\left|\boldsymbol{r}_{p}\right|\right) \frac{d u}{u^{2}\left(u^{2}-1\right)^{1 / 2}}, \\
& I_{1}\left(\left|\boldsymbol{r}_{p}\right|\right)=\int_{1}^{\infty} \mu_{b}\left(u\left|\boldsymbol{r}_{p}\right|\right) \frac{d u}{u^{2}\left(u^{2}-1\right)^{1 / 2}}, \\
& J_{0}\left(\left|\boldsymbol{r}_{p}\right|\right)=\int_{1}^{\infty} \mu_{b}\left(u\left|\boldsymbol{r}_{p}\right|\right) \frac{d u}{u^{4}\left(u^{2}-1\right)^{1 / 2}}, \\
& J_{1}\left(\left|\boldsymbol{r}_{p}\right|\right)=\int_{1}^{\infty} \mu_{b}\left(u\left|\boldsymbol{r}_{p}\right|\right) \frac{d u}{u^{4}\left(u^{2}-1\right)^{1 / 2}},
\end{aligned}
$$

and Eq. (B4) is

$$
\Delta v_{y}=\frac{2 G M_{b} y}{\left|\boldsymbol{v}_{p}\right|\left|\boldsymbol{r}_{p}\right|^{2}}\left[2 I_{0}\left(\left|\boldsymbol{r}_{p}\right|\right)-3 J_{0}\left(\left|\boldsymbol{r}_{p}\right|\right)-I_{1}\left(\left|\boldsymbol{r}_{p}\right|\right)+J_{1}\left(\left|\boldsymbol{r}_{p}\right|\right)\right]
$$

In the same way, Eqs. (B1), (B3) can be written as

$$
\begin{gathered}
\Delta v_{x}=\frac{2 G M_{b} x}{\left|\boldsymbol{v}_{p}\right|\left|\boldsymbol{r}_{p}\right|^{2}}\left[-I_{0}\left(\left|\boldsymbol{r}_{p}\right|\right)+3 J_{0}\left(\left|\boldsymbol{r}_{p}\right|\right)-J_{1}\left(\left|\boldsymbol{r}_{p}\right|\right)\right] \\
\Delta v_{z}=-\frac{2 G M_{b} z}{\left|\boldsymbol{v}_{p}\right|\left|\boldsymbol{r}_{p}\right|^{2}} I_{0}\left(\left|\boldsymbol{r}_{p}\right|\right)
\end{gathered}
$$

Eqs. (B9), (B10), (B11) are the changes of the velocity components obtained by Gnedin et al. (1999a) (see their equation (10)).

Eqs. (B9), (B10) can be reduced as follows. An integration by parts shows that (assuming, as above, that $\left|\boldsymbol{r}_{p}\right| \neq 0$ )

$$
\int_{0}^{\infty} \frac{\mu_{b}(\xi(t))}{\xi^{3}(t)} d t=3\left|\boldsymbol{v}_{p}\right|^{2} \int_{0}^{\infty} \frac{\mu_{b}(\xi(t)) t^{2}}{\xi^{5}(t)} d t-\frac{4 \pi\left|\boldsymbol{v}_{p}\right|^{2}}{M_{b}} \int_{0}^{\infty} \frac{\rho_{b}(\xi(t)) t^{2}}{\xi^{2}(t)} d t
$$

Thus, returning to Eq. (B2) we find the same result as that obtained when the bulge is a point mass, namely 


$$
\Delta v_{y}=0
$$

whereby $2 I_{0}\left(\left|\boldsymbol{r}_{p}\right|\right)-3 J_{0}\left(\left|\boldsymbol{r}_{p}\right|\right)-I_{1}\left(\left|\boldsymbol{r}_{p}\right|\right)+J_{1}\left(\left|\boldsymbol{r}_{p}\right|\right)=0$, from Eq. (B9). Thus, $3 J_{0}\left(\left|\boldsymbol{r}_{p}\right|\right)-$ $I_{0}\left(\left|\boldsymbol{r}_{p}\right|\right)-J_{1}\left(\left|\boldsymbol{r}_{p}\right|\right)=I_{0}\left(\left|\boldsymbol{r}_{p}\right|\right)-I_{1}\left(\left|\boldsymbol{r}_{p}\right|\right)$, and Eq. (B10) reduces to

$$
\Delta v_{x}=\frac{2 G M_{b} x}{\left|\boldsymbol{v}_{p}\right|\left|\boldsymbol{r}_{p}\right|^{2}}\left[I_{0}\left(\left|\boldsymbol{r}_{p}\right|\right)-I_{1}\left(\left|\boldsymbol{r}_{p}\right|\right)\right]
$$

Then only two integrals, $I_{0}\left(\left|\boldsymbol{r}_{p}\right|\right)$ and $I_{1}\left(\left|\boldsymbol{r}_{p}\right|\right)$, are needed to compute the change of the velocity components.

There is another way to write Eq. (B14). Integrating by parts we find

$$
I_{0}\left(\left|\boldsymbol{r}_{p}\right|\right)=1-\frac{4 \pi\left|\boldsymbol{r}_{p}\right|^{3}}{M_{b}} \int_{1}^{\infty} \rho_{b}\left(u\left|\boldsymbol{r}_{p}\right|\right)\left(u^{2}-1\right)^{1 / 2} u d u
$$

and $I_{1}\left(\left|\boldsymbol{r}_{p}\right|\right)$ can be written as

$$
I_{1}\left(\left|\boldsymbol{r}_{p}\right|\right)=\frac{4 \pi\left|\boldsymbol{r}_{p}\right|^{3}}{M_{b}} \int_{1}^{\infty} \frac{\rho_{b}\left(u\left|\boldsymbol{r}_{p}\right|\right) u d u}{\left(u^{2}-1\right)^{1 / 2}}
$$

thus

$$
\Delta v_{x}=\frac{2 G M_{b} x}{\left|\boldsymbol{v}_{p}\right|\left|\boldsymbol{r}_{p}\right|^{2}}\left[1-\frac{4 \pi\left|\boldsymbol{r}_{p}\right|^{3}}{M_{b}} \Im_{2}\left(\left|\boldsymbol{r}_{p}\right|\right)\right]
$$

with

$$
\Im_{2}\left(\left|\boldsymbol{r}_{p}\right|\right)=\int_{1}^{\infty} \frac{\rho_{b}\left(u\left|\boldsymbol{r}_{p}\right|\right) u^{3} d u}{\left(u^{2}-1\right)^{1 / 2}}
$$

In Section 5.1 we call $\Im_{1}\left(\left|\boldsymbol{r}_{p}\right|\right)=I_{0}\left(\left|\boldsymbol{r}_{p}\right|\right)$.

\section{REFERENCES}

Aguilar, L., Hut, P., \& Ostriker, J. P. 1988, ApJ, 335, 720

Allen, C., 1990, Rev. Mexicana Astron. Astrof., 20, 137 
Allen, C., \& Martos, M. A. 1988, Rev. Mexicana Astron. Astrofis., 16, 25

Allen, C., \& Santillán, A. 1991, Rev. Mexicana Astron. Astrofis., 22, 255

Allen, C., \& Santillán, A. 1993, Rev. Mexicana Astron. Astrofis., 25, 39

Anderson, J., \& King, I. R. 2003, AJ, 126, 772

Bedin, L. R., Piotto, G., King, I. R., \& Anderson, J. 2003, AJ, 126, 247

Belokurov, V., Evans, N. W., Irwin, M. J., Hewett, P. C., \& Wilkinson, M. I. 2006, ApJ, 637, L29

Binney, J., \& Merrifield, M., 1998, Galactic Astronomy (Ed. Princeton Series in Astrophysics)

Brosche, P., Odenkirchen, M., \& Geffert, M. 1999, New Astronomy, 4, 133

Caimmi, R., \& Secco, L. 2003, AN, 324, 491

Dinescu, D. I., Girard, T. M., van Altena, W. F., Méndez, R. A., \& López, C. E. 1997, AJ, 114,1014

Dinescu, D. I., Girard, T. M., van Altena, W. F., \& López, C. E. 1999a, AJ, 117, 277

Dinescu, D. I., Girard, T. M., \& van Altena, W. F. 1999b, AJ, 117, 1792

Dinescu, D. I., Majewski, S. R., Girard, T. M., \& Cudworth, K. M. 2000, AJ, 120, 1892

Dinescu, D. I., Majewski, S. R., Girard, T. M., \& Cudworth, K. M. 2001, AJ, 122, 1916

Dinescu, D. I., Girard, T. M., van Altena, W. F., \& López, C. E. 2003, AJ, 125, 1373

Freudenreich, H. T. 1998, ApJ, 492, 495

Gnedin, O. Y., \& Ostriker, J. P. 1997, ApJ, 474, 223

Gnedin, O. Y., \& Ostriker, J. P. 1999, ApJ, 513, 626

Gnedin, O. Y., Hernquist, L., \& Ostriker, J. P. 1999, ApJ, 514, 109

Gnedin, O. Y., Lee, H. M., \& Ostriker, J. P. 1999, ApJ, 522, 935

Harris, W. E. 1996, AJ, 112, 1487

Keenan, D. W., Innanen, K. A., \& House, F. C. 1973, AJ, 78, 173 
Keenan, D.W. \& Innanen, K.A. 1975, AJ, 80, 290.

King, I. R. 1962, AJ, 67, 471

King, I. R. 1966, AJ, 71, 64

King, I. R. 2004, in Penetrating Bars through Masks of Cosmic Dust, eds. D. L. Block, I. Puerari, K. C. Freeman, R. Groess, \& E. K. Block (Dordrecht: Springer), p. 685

Koch, A., Grebel, E.,K., Odenkirchen, M., Martínez-Delgado, D., \& Caldwell, J. A. R. 2004, AJ, 128, 2274

Kundić, T., \& Ostriker, J. P. 1995, ApJ, 438, 702

Leon, S., Meylan, G., \& Combes, F. 2000, A\&A, 359, 907

Long, K., Ostriker, J. P., \& Aguilar, L. 1992, ApJ, 388, 362

Mackey, A. D., \& Gilmore, G. F. 2004, MNRAS, 355, 504

Miyamoto, M., \& Nagai, R. 1975, PASJ, 27533

Odenkirchen, M., \& Grebel, E. K. 2004, Satellites and Tidal Streams, ASPC, 327, 284

Pichardo, B., Martos, M., \& Moreno, E. 2004, ApJ, 582, 230

Poveda, A., \& Allen, C. 1975, ApJ, 197, 155

Press, W. H., Teukolsky, S. A., Vetterling, W. T., \& Flannery, B. P. 1992, Numerical Recipes in Fortran 77: The Art of Scientific Computing (2nd ed.; Cambridge: Cambridge Univ. Press)

Schmidt, M. 1956, Bull. Astron. Inst. Netherlands, 13, 15

Siegel, M. H., Majewski, S. R., Cudworth, K. M., \& Takamiya, M. 2001, AJ, 121, 935

Spitzer, L. 1958, ApJ, 127, 17

Spitzer, L. 1987, Dynamical Evolution of Globular Clusters (Princeton: Princeton Univ. Press)

Symon, K. R. 1971, Mechanics (Addison-Wesley)

Webbink, R. F. 1998, IAU Symp. 126, J. E. Grindlay and A. G. Davis Phillip, Eds. (Kluwer, Dordrecht) p. 49 
This preprint was prepared with the AAS LATEX macros v5.2. 
Table 1. Orbital properties with the axisymmetric potential

\begin{tabular}{|c|c|c|c|c|c|c|c|c|c|c|c|c|}
\hline Cluster & $\begin{array}{l}\left(r_{\min }\right)_{\min } \\
\quad(k p c)\end{array}$ & $\begin{array}{c}<r_{\min }> \\
\quad(k p c)\end{array}$ & $\begin{array}{l}\left(r_{\max }\right)_{\max } \\
(k p c)\end{array}$ & $\begin{array}{c}<r_{\max }> \\
\quad(k p c)\end{array}$ & $\begin{array}{c}\left(|z|_{\max }\right)_{\max } \\
(k p c)\end{array}$ & $\begin{array}{c}<|z|_{\max }> \\
(k p c)\end{array}$ & $<e>$ & $\begin{array}{c}E \\
\left(10 k m s^{-1}\right)^{2}\end{array}$ & $\begin{array}{c}h \\
\left(10 k m s^{-1} k p c\right)\end{array}$ & $\begin{array}{l}r_{K} \\
(p c)\end{array}$ & $\begin{array}{c}r_{*} \\
(p c)\end{array}$ & $\begin{array}{l}r_{L} \\
(p c)\end{array}$ \\
\hline \multirow[t]{3}{*}{ NGC 104} & 5.81 & 5.94 & 7.90 & 7.82 & 3.51 & 3.19 & 0.137 & -1267.51 & 121.39 & 100.1 & 113.6 & 56.1 \\
\hline & 4.96 & 5.10 & 7.84 & 7.79 & 3.17 & 2.76 & 0.209 & -1297.54 & 113.12 & 90.5 & 101.5 & \\
\hline & 6.79 & 6.91 & 7.98 & 7.90 & 3.83 & 3.65 & 0.067 & -1234.49 & 129.82 & 112.0 & 128.1 & \\
\hline \multirow[t]{3}{*}{ NGC 288} & 3.51 & 4.05 & 12.39 & 12.29 & 9.87 & 6.91 & 0.506 & -1160.77 & -66.87 & 34.1 & 34.5 & 33.1 \\
\hline & 1.45 & 2.16 & 12.16 & 11.91 & 9.45 & 5.59 & 0.695 & -1206.96 & -38.65 & 22.3 & 21.1 & \\
\hline & 6.03 & 6.41 & 13.02 & 12.86 & 10.56 & 8.12 & 0.335 & -1101.99 & -95.17 & 46.1 & 49.1 & \\
\hline \multirow[t]{3}{*}{ NGC 362} & 0.50 & 0.78 & 12.39 & 11.95 & 7.09 & 1.51 & 0.878 & -1248.36 & -27.30 & 18.4 & 17.2 & 39.8 \\
\hline & 0.54 & 1.11 & 10.86 & 10.10 & 6.08 & 2.27 & 0.803 & -1309.71 & -28.07 & 18.7 & 18.1 & \\
\hline & 0.54 & 1.40 & 14.17 & 13.60 & 11.37 & 5.76 & 0.816 & -1164.84 & -21.78 & 21.4 & 19.0 & \\
\hline \multirow[t]{3}{*}{ NGC 1851} & 5.76 & 5.88 & 37.53 & 37.50 & 15.18 & 8.50 & 0.729 & -750.45 & 207.16 & 67.7 & 64.2 & 41.2 \\
\hline & 4.59 & 4.73 & 26.31 & 26.28 & 10.05 & 5.83 & 0.695 & -888.71 & 162.17 & 59.5 & 56.9 & \\
\hline & 5.99 & 6.08 & 58.62 & 58.58 & 25.03 & 12.67 & 0.812 & -584.39 & 230.93 & 69.1 & 62.8 & \\
\hline \multirow[t]{3}{*}{ NGC 1904} & 4.06 & 4.29 & 21.58 & 21.54 & 9.95 & 6.02 & 0.668 & -965.01 & 133.40 & 48.2 & 46.3 & 31.3 \\
\hline & 1.57 & 1.88 & 19.49 & 19.40 & 8.21 & 3.91 & 0.823 & -1028.90 & 66.85 & 26.0 & 23.0 & \\
\hline & 9.38 & 9.52 & 24.18 & 24.11 & 14.20 & 9.93 & 0.434 & -873.68 & 225.95 & 84.8 & 87.5 & \\
\hline \multirow[t]{3}{*}{ NGC 2298} & 4.14 & 4.63 & 17.87 & 17.77 & 14.14 & 9.19 & 0.587 & -1024.63 & -85.22 & 31.7 & 31.3 & 20.2 \\
\hline & 0.46 & 1.38 & 16.29 & 15.24 & 12.50 & 5.17 & 0.836 & -1123.79 & -25.23 & 12.3 & 11.2 & \\
\hline & 10.15 & 10.36 & 23.52 & 23.42 & 18.51 & 13.45 & 0.386 & -872.77 & -176.66 & 55.7 & 58.2 & \\
\hline \multirow[t]{3}{*}{ Pal 3} & $\ldots$ & $\ldots$ & $\ldots$ & $\ldots$ & $\ldots$ & $\ldots$ & $\ldots$ & $\ldots$ & $\ldots$ & $\ldots$ & $\ldots$ & 129.7 \\
\hline & 81.92 & 81.92 & 128.27 & 128.26 & 116.90 & 99.24 & 0.220 & -190.11 & 742.66 & 197.3 & 218.4 & \\
\hline & $\ldots$ & $\ldots$ & $\ldots$ & $\ldots$ & $\ldots$ & $\ldots$ & $\ldots$ & $\ldots$ & $\ldots$ & $\ldots$ & $\ldots$ & \\
\hline \multirow[t]{3}{*}{ NGC 4147} & 1.88 & 2.70 & 30.68 & 30.65 & 26.79 & 15.25 & 0.838 & -838.05 & 42.49 & 21.6 & 18.2 & 35.4 \\
\hline & 2.06 & 2.91 & 23.59 & 23.55 & 23.54 & 14.24 & 0.782 & -934.95 & 5.81 & 22.1 & 20.3 & \\
\hline & 9.50 & 9.73 & 46.96 & 46.89 & 41.71 & 25.29 & 0.656 & -651.33 & 154.86 & 51.1 & 49.0 & \\
\hline \multirow[t]{3}{*}{ NGC 4590} & 9.72 & 9.82 & 32.30 & 32.24 & 18.52 & 11.97 & 0.533 & -778.60 & 257.23 & 73.2 & 73.4 & 90.0 \\
\hline & 9.06 & 9.19 & 20.61 & 20.54 & 11.09 & 8.05 & 0.382 & -926.50 & 218.60 & 70.7 & 74.1 & \\
\hline & 10.47 & 10.59 & 52.67 & 52.63 & 32.55 & 19.45 & 0.665 & -608.35 & 298.93 & 76.0 & 73.1 & \\
\hline \multirow[t]{3}{*}{ NGC 5024} & 15.41 & 15.57 & 37.80 & 37.73 & 35.92 & 25.09 & 0.416 & -692.02 & 136.30 & 157.8 & 162.5 & 112.6 \\
\hline & 10.60 & 10.87 & 19.64 & 19.49 & 18.31 & 14.28 & 0.284 & -918.32 & 98.39 & 125.0 & 133.8 & \\
\hline & 17.64 & 17.73 & 108.76 & 108.72 & 105.52 & 60.17 & 0.720 & -336.37 & 161.00 & 168.3 & 156.0 & \\
\hline \multirow[t]{3}{*}{ NGC 5139} & 1.41 & 1.61 & 6.79 & 6.43 & 3.06 & 1.47 & 0.600 & -1488.49 & -48.70 & 56.8 & 54.3 & 87.9 \\
\hline & 0.91 & 1.34 & 6.94 & 6.27 & 3.40 & 1.65 & 0.648 & -1502.10 & -37.34 & 52.6 & 51.5 & \\
\hline & 1.95 & 1.99 & 6.78 & 6.65 & 1.57 & 1.34 & 0.539 & -1468.85 & -60.25 & 70.7 & 69.6 & \\
\hline NGC 5272 & 4.32 & 4.87 & 14.94 & 14.81 & 13.28 & 9.21 & 0.506 & -1081.68 & 61.43 & 76.1 & 77.3 & 115.5 \\
\hline
\end{tabular}


Table 1-Continued

\begin{tabular}{|c|c|c|c|c|c|c|c|c|c|c|c|c|}
\hline Cluster & $\begin{array}{l}\left(r_{\min }\right)_{\min } \\
\quad(k p c)\end{array}$ & $\begin{array}{c}<r_{\min }> \\
\quad(k p c)\end{array}$ & $\begin{array}{l}\left(r_{\max }\right)_{\max } \\
\quad(k p c)\end{array}$ & $\begin{array}{c}<r_{\max }> \\
\quad(k p c)\end{array}$ & $\begin{array}{c}\left(|z|_{\max }\right)_{\max } \\
(k p c)\end{array}$ & $\begin{array}{c}<|z|_{\max }> \\
(k p c)\end{array}$ & $<e>$ & $\begin{array}{c}E \\
\left(10 k m s^{-1}\right)^{2}\end{array}$ & $\begin{array}{c}h \\
\left(10 k m s^{-1} k p c\right)\end{array}$ & $\begin{array}{l}r_{K} \\
(p c)\end{array}$ & $\begin{array}{l}r_{*} \\
(p c)\end{array}$ & $\begin{array}{l}r_{L} \\
(p c)\end{array}$ \\
\hline \multirow{4}{*}{ NGC 5466} & 3.28 & 3.92 & 13.26 & 13.14 & 12.21 & 8.44 & 0.542 & -1136.15 & 39.79 & 64.8 & 64.9 & \\
\hline & 5.65 & 6.10 & 17.46 & 17.32 & 15.23 & 10.58 & 0.480 & -1013.35 & 83.80 & 89.0 & 90.9 & \\
\hline & 6.56 & 6.93 & 71.46 & 71.40 & 70.59 & 40.67 & 0.823 & -507.66 & -43.16 & 50.1 & 44.6 & 158.4 \\
\hline & 3.92 & 4.51 & 37.41 & 37.30 & 35.13 & 20.64 & 0.784 & -755.72 & -49.72 & 37.5 & 33.9 & \\
\hline \multirow{3}{*}{ Pal 5} & 9.54 & 9.72 & 221.50 & 221.47 & 211.42 & 128.57 & 0.916 & -172.69 & -44.86 & 31.6 & 53.4 & \\
\hline & 5.63 & 6.09 & 19.12 & 18.99 & 17.38 & 11.78 & 0.514 & -982.76 & 73.29 & 27.2 & 27.6 & 109.9 \\
\hline & 2.52 & 3.29 & 16.39 & 16.32 & 15.24 & 9.69 & 0.666 & -1068.39 & 34.34 & 17.9 & 17.1 & \\
\hline \multirow{4}{*}{ NGC 5897} & 9.81 & 10.08 & 22.99 & 22.87 & 20.49 & 14.77 & 0.388 & -881.76 & 124.74 & 39.3 & 41.0 & \\
\hline & 1.12 & 1.97 & 9.41 & 9.33 & 8.05 & 5.11 & 0.656 & -1298.83 & 25.39 & 23.8 & 22.7 & 43.5 \\
\hline & 0.04 & 0.57 & 8.56 & 7.51 & 6.62 & 2.25 & 0.863 & -1430.58 & 2.46 & 9.4 & 8.0 & \\
\hline & 3.47 & 4.05 & 14.35 & 14.25 & 12.06 & 8.11 & 0.558 & -1108.43 & 61.80 & 39.1 & 38.3 & \\
\hline \multirow[t]{2}{*}{ NGC 5904} & 1.64 & 2.51 & 49.62 & 49.60 & 45.38 & 24.88 & 0.905 & -654.46 & 33.07 & 42.8 & 35.8 & 62.0 \\
\hline & 0.93 & 1.90 & 30.07 & 30.03 & 26.72 & 14.41 & 0.883 & -849.11 & 23.52 & 35.5 & 30.7 & \\
\hline \multirow{3}{*}{ NGC 6093} & 2.45 & 3.23 & 89.15 & 89.12 & 81.60 & 44.97 & 0.930 & -429.25 & 40.05 & 62.7 & 51.7 & \\
\hline & 2.57 & 2.84 & 3.84 & 3.69 & 3.78 & 3.61 & 0.130 & -1548.60 & 10.34 & 43.8 & 46.7 & 38.6 \\
\hline & 0.02 & 0.54 & 5.04 & 3.97 & 3.61 & 1.69 & 0.766 & -1679.98 & -0.74 & 15.8 & 15.4 & \\
\hline \multirow{3}{*}{ NGC 6121} & 3.44 & 3.93 & 6.25 & 6.03 & 5.83 & 5.09 & 0.213 & -1377.54 & 29.90 & 52.6 & 57.3 & \\
\hline & 0.33 & 0.66 & 6.81 & 6.14 & 4.05 & 1.09 & 0.806 & -1538.14 & -18.19 & 8.6 & 7.9 & 20.8 \\
\hline & 0.04 & 0.57 & 6.60 & 5.56 & 4.76 & 1.87 & 0.824 & -1556.13 & -2.55 & 10.0 & 9.2 & \\
\hline \multirow{3}{*}{ NGC 6144} & 0.78 & 0.92 & 6.96 & 6.66 & 3.36 & 0.72 & 0.757 & -1513.82 & -34.84 & 11.7 & 10.9 & \\
\hline & 2.10 & 2.13 & 2.64 & 2.62 & 2.33 & 2.32 & 0.102 & -1687.21 & -20.82 & 23.7 & 24.3 & 82.2 \\
\hline & 1.07 & 1.41 & 2.94 & 2.90 & 2.53 & 2.08 & 0.351 & -1717.06 & -16.62 & 16.3 & 16.7 & \\
\hline \multirow{3}{*}{ NGC 6171} & 1.46 & 1.88 & 3.83 & 3.79 & 3.42 & 2.81 & 0.343 & -1604.59 & -19.43 & 20.1 & 20.5 & \\
\hline & 1.99 & 2.27 & 3.66 & 3.62 & 2.61 & 2.31 & 0.230 & -1612.79 & 35.84 & 26.3 & 27.9 & 32.5 \\
\hline & 0.96 & 1.35 & 3.63 & 3.55 & 2.78 & 2.11 & 0.454 & -1667.74 & 21.62 & 17.4 & 17.6 & \\
\hline \multirow{3}{*}{ NGC 6205} & 3.32 & 3.47 & 4.00 & 3.89 & 2.54 & 2.49 & 0.058 & -1533.24 & 53.28 & 35.9 & 40.3 & \\
\hline & 5.72 & 6.20 & 30.33 & 30.22 & 28.86 & 17.57 & 0.660 & -822.78 & -60.67 & 80.6 & 79.0 & 56.4 \\
\hline & 4.21 & 4.82 & 19.00 & 18.87 & 18.23 & 11.85 & 0.594 & -998.58 & -39.17 & 69.7 & 69.9 & \\
\hline \multirow{3}{*}{ NGC 6218} & 6.89 & 7.27 & 52.78 & 52.71 & 49.91 & 28.24 & 0.758 & -618.62 & -87.81 & 89.1 & 83.3 & \\
\hline & 2.02 & 2.39 & 5.66 & 5.65 & 3.45 & 2.68 & 0.407 & -1480.98 & 48.45 & 28.1 & 28.9 & 25.1 \\
\hline & 1.33 & 1.74 & 5.35 & 5.24 & 3.32 & 2.38 & 0.505 & -1531.98 & 36.68 & 21.7 & 21.6 & \\
\hline \multirow{3}{*}{ NGC 6254} & 2.72 & 3.05 & 6.37 & 6.32 & 3.74 & 3.02 & 0.350 & -1420.25 & 61.48 & 33.7 & 35.5 & \\
\hline & 3.03 & 3.28 & 5.25 & 5.18 & 2.96 & 2.61 & 0.226 & -1473.24 & 62.12 & 37.9 & 41.6 & 27.5 \\
\hline & 2.07 & 2.38 & 4.77 & 4.76 & 2.81 & 2.31 & 0.335 & -1539.05 & 47.30 & 30.4 & 32.0 & \\
\hline
\end{tabular}


Table 1-Continued

\begin{tabular}{|c|c|c|c|c|c|c|c|c|c|c|c|c|}
\hline Cluster & $\begin{array}{l}\left(r_{\min }\right)_{\min } \\
\quad(k p c)\end{array}$ & $\begin{array}{c}<r_{\min }> \\
\quad(k p c)\end{array}$ & $\begin{array}{l}\left(r_{\max }\right)_{\max } \\
(k p c)\end{array}$ & $\begin{array}{c}<r_{\max }> \\
\quad(k p c)\end{array}$ & $\begin{array}{c}\left(|z|_{\max }\right)_{\max } \\
(k p c)\end{array}$ & $\begin{array}{c}<|z|_{\max }> \\
(k p c)\end{array}$ & $<e>$ & $\begin{array}{c}E \\
\left(10 k m s^{-1}\right)^{2}\end{array}$ & $\begin{array}{c}h \\
\left(10 k m s^{-1} k p c\right)\end{array}$ & $\begin{array}{l}r_{K} \\
(p c)\end{array}$ & $\begin{array}{c}r_{*} \\
(p c)\end{array}$ & $\begin{array}{l}r_{L} \\
(p c)\end{array}$ \\
\hline \multirow{4}{*}{ NGC 6266} & 4.04 & 4.24 & 6.03 & 5.93 & 3.35 & 3.03 & 0.166 & -1395.28 & 78.61 & 44.9 & 50.4 & \multirow{3}{*}{18.0} \\
\hline & 1.19 & 1.36 & 2.61 & 2.36 & 1.05 & 0.85 & 0.269 & -1839.13 & 30.47 & 33.5 & 35.7 & \\
\hline & 0.73 & 0.87 & 1.77 & 1.71 & 1.06 & 0.86 & 0.330 & -1958.02 & 19.50 & 23.6 & 24.6 & \\
\hline & 2.10 & 2.17 & 3.08 & 2.89 & 1.15 & 1.09 & 0.144 & -1719.60 & 43.43 & 48.5 & 53.1 & \\
\hline \multirow[t]{2}{*}{ NGC 6304} & 1.67 & 1.70 & 3.29 & 3.25 & 0.62 & 0.57 & 0.315 & -1745.93 & 43.89 & 23.0 & 24.1 & \multirow[t]{2}{*}{23.1} \\
\hline & 1.25 & 1.26 & 2.48 & 2.41 & 0.64 & 0.58 & 0.313 & -1854.56 & 32.74 & 18.2 & 18.9 & \\
\hline \multirow{4}{*}{ NGC 6316} & 2.12 & 2.15 & 4.18 & 4.16 & 0.62 & 0.57 & 0.319 & -1649.12 & 56.35 & 27.3 & 28.9 & \multirow{3}{*}{19.0} \\
\hline & 0.88 & 0.91 & 3.02 & 2.98 & 1.31 & 0.94 & 0.532 & -1805.20 & -22.35 & 18.3 & 18.8 & \\
\hline & 0.17 & 0.31 & 2.15 & 1.84 & 1.33 & 0.62 & 0.711 & -2027.97 & -8.62 & 9.0 & 8.1 & \\
\hline & 1.78 & 1.89 & 4.61 & 4.47 & 1.87 & 1.42 & 0.405 & -1614.19 & -43.55 & 32.8 & 34.6 & \\
\hline \multirow[t]{3}{*}{ NGC 6341} & 0.27 & 1.02 & 11.51 & 10.74 & 6.92 & 2.25 & 0.828 & -1287.00 & 16.41 & 21.6 & 20.2 & \multirow[t]{2}{*}{36.2} \\
\hline & 1.20 & 1.32 & 9.86 & 9.68 & 4.30 & 2.49 & 0.760 & -1327.62 & 23.71 & 22.7 & 21.6 & \\
\hline & 0.09 & 0.77 & 12.94 & 12.07 & 10.31 & 2.92 & 0.883 & -1232.39 & 5.06 & 19.5 & 17.9 & \\
\hline \multirow[t]{3}{*}{ NGC 6362} & 2.15 & 2.36 & 6.08 & 5.74 & 2.45 & 1.71 & 0.417 & -1502.69 & 58.29 & 25.0 & 26.2 & \multirow[t]{3}{*}{36.8} \\
\hline & 1.89 & 2.01 & 5.52 & 5.36 & 2.21 & 1.61 & 0.454 & -1546.21 & 48.08 & 21.9 & 22.7 & \\
\hline & 2.67 & 2.93 & 6.16 & 6.15 & 2.89 & 2.32 & 0.355 & -1443.96 & 67.17 & 29.3 & 30.9 & \\
\hline \multirow[t]{3}{*}{ NGC 6397} & 2.75 & 2.94 & 6.98 & 6.64 & 2.64 & 1.90 & 0.387 & -1428.45 & 74.27 & 27.4 & 29.2 & \multirow[t]{3}{*}{10.6} \\
\hline & 2.24 & 2.26 & 6.59 & 6.39 & 1.71 & 1.51 & 0.479 & -1469.13 & 64.04 & 22.3 & 22.2 & \\
\hline & 3.35 & 3.55 & 6.99 & 6.97 & 2.85 & 2.31 & 0.326 & -1384.20 & 84.89 & 30.4 & 32.7 & \\
\hline \multirow[t]{3}{*}{ NGC 6522} & 0.67 & 0.96 & 2.93 & 2.78 & 1.98 & 1.43 & 0.492 & -1785.03 & 19.42 & 15.6 & 15.7 & \multirow[t]{3}{*}{37.3} \\
\hline & 0.04 & 0.24 & 1.87 & 1.54 & 1.41 & 0.78 & 0.731 & -2092.40 & -1.92 & 7.7 & 6.9 & \\
\hline & 1.45 & 1.90 & 6.90 & 6.82 & 4.18 & 2.85 & 0.566 & -1432.96 & 42.17 & 26.2 & 25.5 & \\
\hline \multirow[t]{3}{*}{ NGC 6528} & 0.45 & 0.60 & 2.51 & 2.23 & 1.23 & 0.63 & 0.573 & -1931.17 & 17.82 & 7.9 & 8.1 & \multirow[t]{3}{*}{38.1} \\
\hline & 0.00 & 0.33 & 1.85 & 1.44 & 1.48 & 0.85 & 0.638 & -2095.91 & 0.03 & 6.8 & 7.1 & \\
\hline & 0.98 & 1.19 & 5.19 & 4.79 & 2.31 & 0.97 & 0.602 & -1625.27 & 37.10 & 12.3 & 11.9 & \\
\hline \multirow[t]{3}{*}{ NGC 6553} & 2.50 & 2.50 & 13.72 & 13.72 & 0.78 & 0.54 & 0.691 & -1177.35 & 98.58 & 33.8 & 31.0 & \multirow[t]{3}{*}{14.2} \\
\hline & 1.89 & 1.90 & 9.73 & 9.73 & 0.77 & 0.54 & 0.673 & -1334.85 & 72.08 & 27.7 & 25.8 & \\
\hline & 3.10 & 3.10 & 19.12 & 19.12 & 0.95 & 0.57 & 0.721 & -1032.85 & 127.12 & 38.5 & 35.4 & \\
\hline \multirow[t]{3}{*}{ NGC 6584} & 0.52 & 0.85 & 15.06 & 14.57 & 9.00 & 1.46 & 0.891 & -1161.57 & 29.50 & 19.4 & 17.9 & \multirow[t]{3}{*}{36.5} \\
\hline & 0.77 & 0.97 & 8.90 & 8.54 & 5.50 & 2.34 & 0.796 & -1381.18 & 9.76 & 15.0 & 14.2 & \\
\hline & 1.99 & 2.24 & 26.56 & 26.50 & 17.50 & 8.86 & 0.844 & -898.59 & 59.69 & 29.2 & 25.7 & \\
\hline \multirow[t]{3}{*}{ NGC 6626} & 2.00 & 2.02 & 3.14 & 3.11 & 0.65 & 0.62 & 0.213 & -1733.94 & 47.45 & 34.1 & 36.8 & \multirow[t]{3}{*}{18.4} \\
\hline & 1.25 & 1.26 & 2.65 & 2.58 & 0.67 & 0.61 & 0.343 & -1834.98 & 33.46 & 23.4 & 24.2 & \\
\hline & 2.99 & 3.01 & 3.66 & 3.64 & 0.74 & 0.72 & 0.094 & -1630.17 & 63.95 & 45.0 & 51.9 & \\
\hline
\end{tabular}


Table 1-Continued

\begin{tabular}{|c|c|c|c|c|c|c|c|c|c|c|c|c|}
\hline Cluster & $\begin{array}{l}\left(r_{\min }\right)_{\min } \\
\quad(k p c)\end{array}$ & $\begin{array}{c}<r_{\min }> \\
\quad(k p c)\end{array}$ & $\begin{array}{c}\left(r_{\max }\right)_{\max } \\
(k p c)\end{array}$ & $\begin{array}{c}<r_{\max }> \\
\quad(k p c)\end{array}$ & $\begin{array}{c}(|z| \max )_{\max } \\
(k p c)\end{array}$ & $\begin{array}{c}<|z|_{\max }> \\
(k p c)\end{array}$ & $<e>$ & $\begin{array}{c}E \\
\left(10 k m s^{-1}\right)^{2}\end{array}$ & $\begin{array}{c}h \\
\left(10 k m s^{-1} k p c\right)\end{array}$ & $\begin{array}{l}r_{K} \\
(p c)\end{array}$ & $\begin{array}{c}r_{*} \\
(p c)\end{array}$ & $\begin{array}{l}r_{L} \\
(p c)\end{array}$ \\
\hline \multirow[t]{3}{*}{ NGC 6656} & 3.05 & 3.17 & 10.23 & 10.06 & 3.54 & 2.33 & 0.521 & -1274.96 & 91.23 & 48.7 & 49.4 & 27.0 \\
\hline & 2.29 & 2.35 & 8.54 & 8.47 & 1.76 & 1.40 & 0.566 & -1367.13 & 75.02 & 40.1 & 39.4 & \\
\hline & 3.32 & 3.48 & 12.83 & 12.80 & 4.45 & 2.81 & 0.573 & -1173.35 & 108.71 & 51.3 & 51.2 & \\
\hline \multirow[t]{3}{*}{ NGC 6712} & 0.91 & 0.99 & 6.87 & 6.61 & 3.72 & 1.94 & 0.739 & -1490.43 & 11.45 & 14.7 & 14.2 & 14.9 \\
\hline & 0.04 & 0.56 & 6.72 & 5.66 & 4.85 & 1.90 & 0.828 & -1547.13 & -2.59 & 10.0 & 9.2 & \\
\hline & 1.36 & 1.38 & 7.87 & 7.85 & 3.16 & 2.13 & 0.702 & -1415.39 & 27.29 & 19.1 & 18.5 & \\
\hline \multirow[t]{3}{*}{ NGC 6723} & 1.96 & 2.01 & 2.60 & 2.57 & 2.59 & 2.56 & 0.122 & -1690.11 & 2.61 & 31.4 & 31.8 & 26.6 \\
\hline & 1.15 & 1.47 & 2.61 & 2.58 & 2.42 & 2.08 & 0.279 & -1741.99 & 11.96 & 23.1 & 23.6 & \\
\hline & 1.85 & 2.39 & 3.63 & 3.42 & 3.55 & 3.13 & 0.183 & -1598.10 & -10.40 & 34.8 & 37.7 & \\
\hline \multirow[t]{3}{*}{ NGC 6752} & 4.71 & 4.77 & 5.83 & 5.77 & 1.79 & 1.70 & 0.095 & -1402.51 & 100.32 & 51.9 & 60.5 & 64.4 \\
\hline & 4.19 & 4.20 & 5.73 & 5.55 & 1.94 & 1.83 & 0.138 & -1431.89 & 89.33 & 47.7 & 54.7 & \\
\hline & 5.37 & 5.43 & 5.99 & 5.95 & 1.66 & 1.61 & 0.046 & -1370.16 & 111.79 & 56.4 & 66.3 & \\
\hline \multirow[t]{3}{*}{ NGC 6779} & 1.01 & 1.06 & 13.69 & 13.59 & 1.51 & 0.88 & 0.856 & -1197.37 & -46.73 & 15.0 & 13.4 & 25.2 \\
\hline & 0.41 & 0.76 & 12.03 & 11.41 & 6.97 & 1.60 & 0.876 & -1266.17 & -23.36 & 11.3 & 10.1 & \\
\hline & 1.76 & 1.83 & 16.19 & 16.14 & 2.53 & 1.64 & 0.797 & -1111.58 & -74.69 & 23.0 & 20.5 & \\
\hline \multirow[t]{3}{*}{ NGC 6809} & 0.94 & 1.69 & 6.50 & 6.44 & 6.08 & 4.15 & 0.593 & -1436.11 & 12.01 & 22.9 & 22.5 & 25.1 \\
\hline & 1.13 & 1.80 & 5.68 & 5.63 & 5.25 & 3.78 & 0.523 & -1479.90 & 14.89 & 23.8 & 23.6 & \\
\hline & 0.71 & 1.54 & 7.60 & 7.52 & 7.38 & 4.83 & 0.669 & -1380.69 & -1.74 & 20.1 & 19.2 & \\
\hline \multirow[t]{3}{*}{ NGC 6838} & 4.64 & 4.64 & 7.15 & 7.15 & 0.32 & 0.29 & 0.213 & -1369.37 & 121.59 & 26.3 & 30.0 & 10.4 \\
\hline & 3.94 & 3.94 & 7.14 & 7.14 & 0.35 & 0.31 & 0.289 & -1396.93 & 109.31 & 23.9 & 26.3 & \\
\hline & 5.28 & 5.28 & 7.36 & 7.36 & 0.37 & 0.34 & 0.164 & -1335.35 & 133.28 & 28.8 & 33.0 & \\
\hline \multirow[t]{3}{*}{ NGC 6934} & 6.91 & 7.20 & 56.01 & 55.93 & 54.62 & 31.37 & 0.772 & -597.11 & -59.29 & 58.7 & 53.9 & 38.2 \\
\hline & 2.33 & 3.14 & 26.49 & 26.43 & 26.23 & 15.72 & 0.788 & -890.30 & -12.96 & 33.8 & 30.1 & \\
\hline & 9.95 & 10.08 & 211.01 & 210.97 & 200.10 & 114.78 & 0.909 & -181.02 & -110.29 & 75.3 & 64.2 & \\
\hline \multirow[t]{3}{*}{ NGC 7006} & 18.25 & 18.28 & 111.35 & 111.34 & 56.13 & 31.57 & 0.718 & -328.03 & 600.50 & 122.4 & 114.2 & 76.5 \\
\hline & 6.84 & 6.93 & 56.53 & 56.51 & 21.67 & 11.62 & 0.782 & -595.58 & 262.88 & 61.5 & 57.0 & \\
\hline & $\ldots$ & $\ldots$ & $\ldots$ & $\ldots$ & $\ldots$ & $\ldots$ & $\ldots$ & $\ldots$ & $\ldots$ & $\ldots$ & $\ldots$ & \\
\hline \multirow[t]{3}{*}{ NGC 7078} & 6.15 & 6.41 & 11.96 & 11.84 & 7.68 & 6.10 & 0.298 & -1131.02 & 124.56 & 97.6 & 105.6 & 64.4 \\
\hline & 4.00 & 4.29 & 10.51 & 10.44 & 5.77 & 4.31 & 0.418 & -1220.05 & 98.49 & 74.4 & 77.8 & \\
\hline & 8.76 & 8.98 & 14.86 & 14.73 & 10.42 & 8.49 & 0.243 & -1022.28 & 156.89 & 124.1 & 134.8 & \\
\hline \multirow[t]{3}{*}{ NGC 7089} & 6.59 & 6.99 & 43.52 & 43.44 & 41.47 & 23.79 & 0.723 & -689.58 & -75.45 & 96.4 & 89.8 & 71.8 \\
\hline & 5.59 & 6.10 & 23.64 & 23.51 & 23.12 & 14.84 & 0.588 & -910.25 & -38.70 & 88.2 & 86.9 & \\
\hline & 8.48 & 8.72 & 90.69 & 90.64 & 84.72 & 47.39 & 0.824 & -415.82 & -127.73 & 112.1 & 98.1 & \\
\hline NGC 7099 & 3.62 & 4.08 & 7.66 & 7.51 & 6.15 & 5.03 & 0.297 & -1317.26 & -56.76 & 42.5 & 45.7 & 42.7 \\
\hline
\end{tabular}


Table 1-Continued

\begin{tabular}{|c|c|c|c|c|c|c|c|c|c|c|c|c|}
\hline Cluster & $\begin{array}{c}\left(r_{\min }\right)_{\min } \\
\quad(k p c)\end{array}$ & $\begin{array}{c}<r_{\min }> \\
(k p c)\end{array}$ & $\begin{array}{c}\left(r_{\max }\right)_{\max } \\
(k p c)\end{array}$ & $\begin{array}{c}<r_{\max }> \\
(k p c)\end{array}$ & $\begin{array}{c}\left(|z|_{\max }\right)_{\max } \\
(k p c)\end{array}$ & $\begin{array}{c}<|z|_{\max }> \\
(k p c)\end{array}$ & $<e>$ & $\begin{array}{c}E \\
\left(10 k m s^{-1}\right)^{2}\end{array}$ & $\begin{array}{c}h \\
\left(10 k m s^{-1} k p c\right)\end{array}$ & $\begin{array}{l}r_{K} \\
(p c)\end{array}$ & $\begin{array}{c}r_{*} \\
(p c)\end{array}$ & $\begin{array}{l}r_{L} \\
(p c)\end{array}$ \\
\hline & 1.96 & 2.48 & 7.10 & 7.06 & 5.36 & 3.94 & 0.483 & -1390.93 & -40.88 & 29.9 & 30.1 & \\
\hline & 4.99 & 5.41 & 9.40 & 9.21 & 7.81 & 6.43 & 0.260 & -1219.80 & -70.12 & 52.1 & 56.9 & \\
\hline \multirow[t]{3}{*}{ Pal 12} & 15.45 & 15.60 & 26.24 & 26.14 & 23.45 & 18.71 & 0.253 & -794.28 & 172.52 & 44.0 & 47.4 & 96.8 \\
\hline & 13.60 & 13.78 & 15.56 & 15.41 & 14.05 & 13.35 & 0.056 & -939.10 & 122.35 & 40.9 & 45.7 & \\
\hline & 17.19 & 17.30 & 46.10 & 46.05 & 40.87 & 27.77 & 0.454 & -623.07 & 235.93 & 45.5 & 46.9 & \\
\hline \multirow[t]{3}{*}{ Pal 13} & 12.50 & 12.61 & 95.58 & 95.55 & 69.88 & 40.50 & 0.767 & -390.18 & -339.94 & 28.1 & 25.9 & 23.3 \\
\hline & 9.73 & 9.90 & 57.78 & 57.73 & 43.74 & 25.04 & 0.707 & -577.84 & -238.62 & 24.4 & 22.9 & \\
\hline & 15.17 & 15.22 & 211.62 & 211.60 & 149.51 & 86.54 & 0.866 & -178.16 & -457.11 & 32.0 & 28.3 & \\
\hline
\end{tabular}


Table 2. Orbital properties with the non-axisymmetric potential

\begin{tabular}{|c|c|c|c|c|c|c|c|c|c|c|c|}
\hline Cluster & $\begin{array}{l}\left(r_{\min }\right)_{\min } \\
(k p c)\end{array}$ & $\begin{array}{c}<r_{\min }> \\
(k p c)\end{array}$ & $\begin{array}{c}\left(r_{\max }\right)_{\max } \\
(k p c)\end{array}$ & $\begin{array}{c}<r_{\max }> \\
(k p c)\end{array}$ & $\begin{array}{c}\left(|z|_{\max }\right)_{\max } \\
(k p c)\end{array}$ & $\begin{array}{c}<|z|_{\max }> \\
(k p c)\end{array}$ & $<e>$ & $\begin{array}{c}h_{\min } \\
\left(10 k m s^{-1} k p c\right)\end{array}$ & $\begin{array}{c}h_{\max } \\
\left(10 k m s^{-1} k p c\right)\end{array}$ & $\begin{array}{l}r_{K} \\
(p c)\end{array}$ & $\begin{array}{l}r_{*} \\
(p c)\end{array}$ \\
\hline \multirow[t]{3}{*}{ NGC 104} & 5.74 & 5.88 & 8.11 & 7.86 & 3.56 & 3.16 & 0.145 & 119.27 & 123.16 & 99.5 & 112.2 \\
\hline & 4.90 & 5.07 & 8.29 & 7.93 & 3.31 & 2.78 & 0.220 & 111.03 & 116.46 & 89.8 & 100.0 \\
\hline & 6.72 & 6.87 & 8.11 & 7.99 & 3.89 & 3.68 & 0.076 & 129.25 & 131.10 & 111.6 & 127.1 \\
\hline \multirow[t]{3}{*}{ NGC 288} & 3.50 & 4.08 & 13.63 & 12.86 & 11.48 & 7.33 & 0.519 & -67.37 & -60.76 & 33.1 & 33.5 \\
\hline & 1.05 & 2.27 & 16.52 & 13.34 & 14.61 & 7.16 & 0.710 & -40.15 & -17.16 & 22.6 & 21.3 \\
\hline & 6.05 & 6.42 & 13.23 & 12.94 & 10.81 & 8.20 & 0.337 & -95.27 & -94.30 & 46.1 & 49.3 \\
\hline \multirow[t]{3}{*}{ NGC 362} & 0.52 & 1.50 & 13.14 & 11.99 & 10.74 & 5.31 & 0.781 & -33.82 & -15.53 & 25.3 & 22.2 \\
\hline & 0.82 & 1.56 & 10.06 & 8.49 & 6.05 & 2.82 & 0.690 & -44.22 & -27.95 & 25.0 & 23.0 \\
\hline & 0.61 & 1.32 & 14.13 & 13.37 & 9.65 & 4.81 & 0.822 & -28.31 & -20.00 & 25.6 & 21.6 \\
\hline \multirow{3}{*}{ NGC 1851} & 5.76 & 5.89 & 38.46 & 37.68 & 15.23 & 8.48 & 0.730 & 206.29 & 208.77 & 67.5 & 64.0 \\
\hline & 4.60 & 4.75 & 27.57 & 26.82 & 10.49 & 5.92 & 0.699 & 161.67 & 165.26 & 59.0 & 56.3 \\
\hline & 5.97 & 6.07 & 60.31 & 59.58 & 25.57 & 12.85 & 0.815 & 230.82 & 232.75 & 68.6 & 62.4 \\
\hline \multirow[t]{3}{*}{ NGC 1904} & 4.02 & 4.27 & 22.05 & 20.29 & 9.72 & 5.88 & 0.652 & 124.73 & 134.84 & 47.8 & 46.1 \\
\hline & 1.30 & 1.94 & 27.58 & 20.11 & 9.39 & 3.85 & 0.823 & 51.65 & 90.42 & 27.6 & 24.5 \\
\hline & 9.37 & 9.52 & 24.27 & 24.14 & 14.24 & 9.94 & 0.434 & 225.77 & 226.22 & 84.5 & 87.2 \\
\hline \multirow[t]{3}{*}{ NGC 2298} & 4.15 & 4.64 & 17.90 & 17.66 & 14.20 & 9.16 & 0.585 & -86.75 & -84.74 & 31.7 & 31.4 \\
\hline & 0.53 & 1.30 & 17.90 & 15.03 & 12.42 & 4.75 & 0.842 & -33.48 & -15.54 & 14.3 & 12.5 \\
\hline & 10.15 & 10.36 & 23.52 & 23.42 & 18.51 & 13.44 & 0.387 & -176.75 & -176.59 & 55.7 & 58.1 \\
\hline \multirow[t]{3}{*}{ Pal 3} & $\ldots$ & $\ldots$ & $\ldots$ & $\ldots$ & $\ldots$ & $\ldots$ & $\ldots$ & $\ldots$ & $\ldots$ & $\ldots$ & $\ldots$ \\
\hline & 81.92 & 81.92 & 128.27 & 128.26 & 116.90 & 99.24 & 0.220 & 742.66 & 742.66 & 197.3 & 218.4 \\
\hline & $\ldots$ & $\cdots$ & $\ldots$ & $\cdots$ & $\ldots$ & $\cdots$ & & & $\cdots$ & & $\cdots$ \\
\hline \multirow[t]{3}{*}{ NGC 4147} & 2.03 & 2.81 & 38.80 & 31.55 & 31.42 & 16.01 & 0.834 & 32.13 & 57.50 & 21.8 & 18.8 \\
\hline & 2.13 & 3.01 & 26.53 & 24.17 & 25.77 & 14.57 & 0.780 & 0.66 & 13.28 & 22.9 & 20.9 \\
\hline & 9.50 & 9.73 & 46.92 & 46.79 & 41.59 & 25.25 & 0.655 & 154.58 & 154.90 & 51.1 & 48.5 \\
\hline \multirow[t]{3}{*}{ NGC 4590} & 9.70 & 9.82 & 32.49 & 32.31 & 18.62 & 11.98 & 0.534 & 257.06 & 257.49 & 73.0 & 73.3 \\
\hline & 8.98 & 9.14 & 21.32 & 20.86 & 11.29 & 8.13 & 0.390 & 218.43 & 220.35 & 70.5 & 73.7 \\
\hline & 10.47 & 10.58 & 52.92 & 52.75 & 32.68 & 19.48 & 0.666 & 298.83 & 299.16 & 75.9 & 73.0 \\
\hline \multirow[t]{3}{*}{ NGC 5024} & 15.42 & 15.57 & 37.81 & 37.73 & 35.92 & 25.09 & 0.416 & 136.27 & 136.33 & 157.8 & 162.5 \\
\hline & 10.61 & 10.88 & 19.64 & 19.48 & 18.31 & 14.28 & 0.283 & 98.21 & 98.52 & 125.0 & 133.9 \\
\hline & 17.64 & 17.73 & 108.80 & 108.75 & 105.54 & 57.04 & 0.720 & 160.99 & 161.02 & 168.3 & 156.0 \\
\hline \multirow[t]{3}{*}{ NGC 5139} & 1.14 & 1.70 & 8.39 & 6.66 & 3.63 & 1.79 & 0.591 & -55.83 & -34.28 & 68.7 & 69.7 \\
\hline & 0.46 & 1.34 & 8.83 & 6.77 & 5.51 & 2.20 & 0.667 & -45.37 & -15.24 & 59.2 & 57.9 \\
\hline & 1.82 & 2.28 & 7.53 & 6.32 & 3.19 & 1.71 & 0.469 & -71.63 & -53.09 & 75.3 & 77.9 \\
\hline NGC 5272 & 4.27 & 4.85 & 16.48 & 15.35 & 14.35 & 9.32 & 0.520 & 59.24 & 67.26 & 75.6 & 76.4 \\
\hline
\end{tabular}


Table 2-Continued

\begin{tabular}{|c|c|c|c|c|c|c|c|c|c|c|c|}
\hline Cluster & $\begin{array}{l}\left(r_{\min }\right)_{\min } \\
\quad(k p c)\end{array}$ & $\begin{array}{c}<r_{\min }> \\
\quad(k p c)\end{array}$ & $\begin{array}{l}\left(r_{\max }\right)_{\max } \\
(k p c)\end{array}$ & $\begin{array}{c}<r_{\max }> \\
\quad(k p c)\end{array}$ & $\begin{array}{c}(|z| \max )_{\max } \\
(k p c)\end{array}$ & $\begin{array}{c}<|z|_{\max }> \\
\quad(k p c)\end{array}$ & $<e>$ & $\begin{array}{c}h_{\min } \\
\left(10 k m s^{-1} k p c\right)\end{array}$ & $\begin{array}{c}h_{\max } \\
\left(10 k m s^{-1} k p c\right)\end{array}$ & $\begin{array}{l}r_{K} \\
(p c)\end{array}$ & $\begin{array}{c}r_{*} \\
(p c)\end{array}$ \\
\hline \multirow{4}{*}{ NGC 5466} & 3.12 & 3.91 & 15.23 & 13.23 & 13.49 & 8.41 & 0.543 & 26.56 & 48.44 & 65.2 & 65.9 \\
\hline & 5.64 & 6.09 & 17.47 & 17.14 & 15.17 & 10.45 & 0.476 & 82.35 & 84.10 & 88.7 & 90.7 \\
\hline & 6.57 & 6.94 & 71.51 & 71.00 & 70.31 & 40.45 & 0.822 & -43.90 & -43.01 & 50.2 & 44.7 \\
\hline & 3.89 & 4.51 & 37.42 & 36.74 & 34.94 & 20.29 & 0.782 & -51.79 & -49.61 & 37.5 & 34.1 \\
\hline \multirow{3}{*}{ Pal 5} & 9.54 & 9.72 & 221.26 & 221.03 & 210.93 & 128.25 & 0.915 & -44.96 & -44.82 & 31.7 & 53.5 \\
\hline & 5.65 & 6.12 & 19.17 & 18.70 & 17.42 & 11.64 & 0.507 & 71.33 & 73.71 & 27.3 & 27.7 \\
\hline & 2.55 & 3.33 & 16.83 & 16.24 & 15.39 & 9.84 & 0.661 & 31.58 & 35.99 & 18.2 & 17.4 \\
\hline \multirow{3}{*}{ NGC 5897} & 9.82 & 10.08 & 22.99 & 22.84 & 20.45 & 14.76 & 0.387 & 124.53 & 124.82 & 39.3 & 41.0 \\
\hline & 1.33 & 2.26 & 12.37 & 10.48 & 8.92 & 5.63 & 0.647 & 24.46 & 44.22 & 25.5 & 24.2 \\
\hline & 0.05 & 1.00 & 8.29 & 6.90 & 7.21 & 3.09 & 0.759 & -15.47 & 17.60 & 14.0 & 12.1 \\
\hline \multirow{3}{*}{ NGC 5904} & 3.50 & 4.14 & 18.74 & 15.72 & 14.25 & 8.56 & 0.582 & 60.98 & 77.85 & 39.1 & 38.4 \\
\hline & 1.71 & 2.51 & 55.28 & 51.05 & 47.74 & 25.95 & 0.906 & 28.18 & 39.95 & 44.5 & 37.0 \\
\hline & 0.54 & 1.66 & 31.80 & 26.46 & 26.74 & 13.91 & 0.883 & 5.27 & 27.76 & 38.6 & 33.1 \\
\hline \multirow{3}{*}{ NGC 6093} & 2.44 & 3.21 & 100.45 & 94.46 & 88.25 & 48.32 & 0.935 & 38.98 & 47.55 & 63.4 & 52.1 \\
\hline & 1.36 & 2.79 & 5.04 & 3.73 & 4.71 & 3.56 & 0.149 & -7.57 & 23.62 & 43.3 & 47.8 \\
\hline & 0.87 & 1.53 & 3.30 & 2.95 & 3.23 & 2.55 & 0.327 & -10.87 & 0.53 & 29.6 & 31.5 \\
\hline \multirow{3}{*}{ NGC 6121} & 3.41 & 4.05 & 6.47 & 5.75 & 6.00 & 5.02 & 0.174 & 24.31 & 32.75 & 53.3 & 58.9 \\
\hline & 0.43 & 0.92 & 6.86 & 5.81 & 4.55 & 1.75 & 0.728 & -25.55 & -10.73 & 11.8 & 9.9 \\
\hline & 0.08 & 0.87 & 6.82 & 5.02 & 5.44 & 2.12 & 0.711 & -18.80 & 12.03 & 9.0 & 6.4 \\
\hline \multirow{3}{*}{ NGC 6144} & 0.19 & 1.09 & 10.16 & 6.70 & 6.55 & 1.24 & 0.715 & -44.32 & -4.40 & 16.5 & 15.2 \\
\hline & 1.95 & 2.23 & 3.13 & 2.88 & 2.95 & 2.60 & 0.127 & -21.88 & -15.18 & 24.1 & 26.0 \\
\hline & 0.02 & 1.11 & 5.06 & 3.73 & 4.30 & 2.30 & 0.543 & -22.46 & 8.22 & 19.8 & 21.4 \\
\hline \multirow{3}{*}{ NGC 6171} & 0.39 & 1.64 & 5.76 & 4.83 & 5.73 & 3.19 & 0.505 & -21.05 & 3.89 & 22.9 & 24.2 \\
\hline & 1.22 & 2.85 & 6.54 & 4.58 & 3.47 & 2.58 & 0.232 & 11.71 & 71.96 & 27.8 & 31.2 \\
\hline & 1.10 & 2.10 & 6.61 & 3.79 & 3.79 & 2.49 & 0.283 & 8.24 & 64.74 & 22.2 & 24.2 \\
\hline \multirow{3}{*}{ NGC 6205} & 1.00 & 2.46 & 5.40 & 3.30 & 2.96 & 2.16 & 0.159 & 11.42 & 68.48 & 32.2 & 36.9 \\
\hline & 5.74 & 6.22 & 30.21 & 29.98 & 28.62 & 17.32 & 0.657 & -61.63 & -60.46 & 80.9 & 79.3 \\
\hline & 4.24 & 4.89 & 18.85 & 18.34 & 17.90 & 11.53 & 0.580 & -42.19 & -38.96 & 70.2 & 70.6 \\
\hline \multirow{3}{*}{ NGC 6218} & 6.91 & 7.28 & 52.93 & 52.77 & 50.02 & 28.27 & 0.758 & -87.90 & -87.49 & 89.0 & 83.3 \\
\hline & 1.71 & 2.53 & 10.32 & 6.19 & 4.20 & 2.66 & 0.417 & 33.79 & 85.64 & 31.5 & 34.1 \\
\hline & 0.14 & 1.12 & 6.97 & 4.37 & 3.20 & 2.00 & 0.608 & -7.15 & 48.77 & 23.9 & 24.3 \\
\hline \multirow{3}{*}{ NGC 6254} & 2.78 & 3.38 & 10.38 & 8.19 & 4.78 & 3.30 & 0.409 & 60.03 & 91.64 & 33.6 & 36.2 \\
\hline & 0.75 & 3.07 & 7.12 & 5.55 & 3.40 & 2.47 & 0.291 & 18.09 & 80.59 & 38.3 & 42.6 \\
\hline & 0.73 & 1.92 & 6.97 & 4.81 & 3.24 & 1.78 & 0.430 & 16.42 & 70.69 & 34.2 & 37.1 \\
\hline
\end{tabular}


Table 2-Continued

\begin{tabular}{|c|c|c|c|c|c|c|c|c|c|c|c|}
\hline Cluster & $\begin{array}{l}\left(r_{\min }\right)_{\min } \\
\quad(k p c)\end{array}$ & $\begin{array}{c}<r_{\min }> \\
\quad(k p c)\end{array}$ & $\begin{array}{l}\left(r_{\max }\right)_{\max } \\
(k p c)\end{array}$ & $\begin{array}{c}<r_{\max }> \\
\quad(k p c)\end{array}$ & $\begin{array}{c}(|z| \max )_{\max } \\
(k p c)\end{array}$ & $\begin{array}{c}<|z|_{\max }> \\
\quad(k p c)\end{array}$ & $<e>$ & $\begin{array}{c}h_{\min } \\
\left(10 k m s^{-1} k p c\right)\end{array}$ & $\begin{array}{c}h_{\max } \\
\left(10 k m s^{-1} k p c\right)\end{array}$ & $\begin{array}{l}r_{K} \\
(p c)\end{array}$ & $\begin{array}{c}r_{*} \\
(p c)\end{array}$ \\
\hline \multirow{4}{*}{ NGC 6266} & 3.73 & 4.31 & 9.32 & 7.29 & 4.27 & 3.22 & 0.251 & 76.27 & 100.81 & 43.5 & 47.7 \\
\hline & 0.62 & 1.44 & 3.11 & 2.50 & 1.15 & 0.89 & 0.278 & 15.94 & 49.27 & 42.1 & 48.6 \\
\hline & 0.62 & 1.16 & 2.30 & 1.89 & 1.21 & 0.98 & 0.250 & 10.61 & 35.26 & 34.6 & 39.5 \\
\hline & 1.32 & 2.87 & 5.46 & 3.81 & 1.47 & 1.15 & 0.147 & 27.96 & 87.40 & 58.3 & 66.4 \\
\hline \multirow[t]{3}{*}{ NGC 6304} & 1.42 & 3.40 & 7.02 & 5.14 & 1.04 & 0.75 & 0.203 & 32.96 & 105.75 & 31.9 & 35.9 \\
\hline & 1.03 & 1.74 & 2.95 & 2.58 & 0.68 & 0.61 & 0.205 & 23.86 & 55.16 & 23.8 & 27.6 \\
\hline & 1.63 & 2.38 & 5.06 & 4.24 & 0.68 & 0.59 & 0.287 & 40.70 & 77.09 & 29.8 & 33.0 \\
\hline \multirow[t]{3}{*}{ NGC 6316} & 0.13 & 0.78 & 5.07 & 3.37 & 2.98 & 0.89 & 0.616 & -34.43 & 18.64 & 20.3 & 20.8 \\
\hline & 0.02 & 0.49 & 2.84 & 1.96 & 1.69 & 0.81 & 0.610 & -14.97 & 20.38 & 15.0 & 13.6 \\
\hline & 1.58 & 1.75 & 5.00 & 4.64 & 2.17 & 1.44 & 0.451 & -43.02 & -38.34 & 31.6 & 34.1 \\
\hline \multirow[t]{3}{*}{ NGC 6341} & 0.25 & 1.00 & 12.05 & 10.61 & 6.69 & 2.53 & 0.830 & 0.40 & 24.32 & 24.1 & 22.0 \\
\hline & 0.85 & 1.21 & 10.02 & 9.30 & 4.53 & 2.35 & 0.770 & 5.86 & 28.35 & 24.2 & 22.6 \\
\hline & 0.02 & 0.84 & 14.76 & 11.15 & 7.66 & 2.58 & 0.861 & -14.16 & 19.51 & 21.4 & 19.0 \\
\hline \multirow[t]{3}{*}{ NGC 6362} & 0.56 & 1.72 & 7.90 & 5.59 & 2.45 & 1.19 & 0.530 & 10.22 & 80.81 & 20.7 & 22.1 \\
\hline & 0.65 & 1.53 & 6.00 & 4.64 & 2.52 & 1.51 & 0.509 & 14.00 & 55.03 & 18.8 & 20.0 \\
\hline & 2.49 & 3.28 & 10.17 & 7.71 & 4.23 & 2.64 & 0.397 & 60.29 & 98.70 & 28.0 & 29.9 \\
\hline \multirow[t]{3}{*}{ NGC 6397} & 2.62 & 3.00 & 10.38 & 7.33 & 3.02 & 1.90 & 0.410 & 58.18 & 103.30 & 27.4 & 29.6 \\
\hline & 1.98 & 2.76 & 7.64 & 6.20 & 3.25 & 2.04 & 0.384 & 48.31 & 82.06 & 25.1 & 26.5 \\
\hline & 2.90 & 3.45 & 9.89 & 8.07 & 3.59 & 2.10 & 0.396 & 76.62 & 104.50 & 30.8 & 33.0 \\
\hline \multirow[t]{3}{*}{ NGC 6522} & 0.35 & 1.84 & 8.23 & 5.97 & 3.20 & 1.61 & 0.535 & 4.87 & 76.17 & 16.8 & 16.0 \\
\hline & 0.05 & 1.07 & 2.64 & 2.38 & 2.40 & 1.95 & 0.394 & -7.47 & 17.36 & 16.2 & 15.5 \\
\hline & 0.96 & 1.82 & 7.25 & 5.85 & 4.13 & 2.63 & 0.527 & 23.48 & 51.61 & 28.6 & 28.9 \\
\hline \multirow[t]{3}{*}{ NGC 6528} & 0.01 & 0.47 & 3.41 & 2.45 & 1.92 & 0.84 & 0.684 & -16.74 & 19.04 & 7.4 & 5.5 \\
\hline & 0.13 & 0.94 & 4.15 & 3.03 & 2.06 & 1.27 & 0.537 & -7.01 & 36.73 & 10.4 & 10.0 \\
\hline & 0.68 & 1.94 & 6.76 & 4.30 & 2.70 & 1.62 & 0.383 & 12.41 & 71.22 & 17.2 & 17.9 \\
\hline \multirow[t]{3}{*}{ NGC 6553} & 2.42 & 2.50 & 12.40 & 11.42 & 0.92 & 0.51 & 0.640 & 86.63 & 104.83 & 33.2 & 32.0 \\
\hline & 1.45 & 1.80 & 8.63 & 7.15 & 1.62 & 0.82 & 0.598 & 47.11 & 78.60 & 27.7 & 26.9 \\
\hline & 3.05 & 3.15 & 19.94 & 17.60 & 0.92 & 0.53 & 0.695 & 117.72 & 135.78 & 38.5 & 36.1 \\
\hline \multirow[t]{3}{*}{ NGC 6584} & 0.90 & 1.52 & 15.33 & 14.63 & 9.13 & 4.98 & 0.813 & 29.86 & 38.39 & 23.3 & 20.9 \\
\hline & 0.11 & 0.83 & 10.91 & 8.11 & 5.74 & 2.04 & 0.814 & -8.08 & 24.37 & 13.4 & 10.9 \\
\hline & 1.75 & 2.20 & 30.42 & 25.74 & 18.73 & 8.66 & 0.841 & 46.10 & 68.94 & 30.1 & 26.3 \\
\hline \multirow[t]{3}{*}{ NGC 6626} & 0.95 & 1.75 & 3.30 & 2.84 & 0.67 & 0.51 & 0.246 & 24.65 & 63.47 & 31.3 & 36.2 \\
\hline & 0.91 & 1.48 & 2.69 & 2.34 & 0.64 & 0.56 & 0.235 & 21.63 & 50.44 & 27.8 & 32.2 \\
\hline & 1.06 & 3.40 & 6.72 & 4.64 & 0.99 & 0.74 & 0.157 & 27.51 & 106.52 & 42.8 & 49.6 \\
\hline
\end{tabular}


Table 2-Continued

\begin{tabular}{|c|c|c|c|c|c|c|c|c|c|c|c|}
\hline Cluster & $\begin{array}{l}\left(r_{\min }\right)_{\min } \\
\quad(k p c)\end{array}$ & $\begin{array}{c}<r_{\min }> \\
\quad(k p c)\end{array}$ & $\begin{array}{c}\left(r_{\max }\right)_{\max } \\
(k p c)\end{array}$ & $\begin{array}{c}<r_{\max }> \\
\quad(k p c)\end{array}$ & $\begin{array}{c}\left(|z|_{\max }\right)_{\max } \\
(k p c)\end{array}$ & $\begin{array}{c}<|z|_{\max }> \\
(k p c)\end{array}$ & $<e>$ & $\begin{array}{c}h_{\min } \\
\left(10 k m s^{-1} k p c\right)\end{array}$ & $\begin{array}{c}h_{\max } \\
\left(10 k m s^{-1} k p c\right)\end{array}$ & $\begin{array}{l}r_{K} \\
(p c)\end{array}$ & $\begin{array}{c}r_{*} \\
(p c)\end{array}$ \\
\hline \multirow[t]{3}{*}{ NGC 6656} & 2.44 & 3.04 & 10.33 & 8.40 & 3.87 & 2.36 & 0.466 & 64.32 & 94.18 & 45.3 & 47.6 \\
\hline & 2.03 & 2.80 & 10.71 & 7.90 & 4.15 & 2.17 & 0.473 & 58.74 & 95.05 & 39.4 & 40.3 \\
\hline & 3.17 & 3.43 & 14.55 & 12.62 & 4.96 & 2.56 & 0.571 & 98.25 & 116.90 & 51.4 & 52.0 \\
\hline \multirow[t]{3}{*}{ NGC 6712} & 0.08 & 0.88 & 9.83 & 7.08 & 4.78 & 1.76 & 0.782 & -1.26 & 43.46 & 16.8 & 16.0 \\
\hline & 0.04 & 0.83 & 7.36 & 6.35 & 3.97 & 1.70 & 0.771 & -8.50 & 13.91 & 15.3 & 14.3 \\
\hline & 0.16 & 0.90 & 9.80 & 7.14 & 4.16 & 1.47 & 0.780 & -2.57 & 46.43 & 20.7 & 19.9 \\
\hline \multirow[t]{3}{*}{ NGC 6723} & 1.24 & 2.17 & 3.46 & 3.06 & 3.33 & 2.90 & 0.175 & -0.97 & 18.18 & 32.1 & 35.2 \\
\hline & 1.33 & 1.99 & 2.93 & 2.61 & 2.54 & 2.34 & 0.138 & 7.85 & 23.55 & 30.4 & 33.2 \\
\hline & 1.10 & 2.39 & 7.82 & 5.23 & 6.41 & 4.20 & 0.372 & -15.42 & 30.00 & 33.3 & 35.7 \\
\hline \multirow[t]{3}{*}{ NGC 6752} & 4.36 & 4.54 & 8.96 & 7.28 & 2.35 & 1.80 & 0.227 & 99.10 & 120.82 & 48.7 & 53.4 \\
\hline & 3.91 & 4.13 & 6.75 & 5.88 & 2.13 & 1.84 & 0.174 & 86.83 & 98.76 & 46.4 & 52.1 \\
\hline & 4.92 & 5.10 & 8.11 & 7.00 & 1.99 & 1.66 & 0.154 & 110.79 & 123.83 & 53.4 & 60.7 \\
\hline \multirow[t]{3}{*}{ NGC 6779} & 0.97 & 1.44 & 14.56 & 12.90 & 7.21 & 2.12 & 0.798 & -65.00 & -41.25 & 17.2 & 14.6 \\
\hline & 0.15 & 0.73 & 14.96 & 12.19 & 7.84 & 1.34 & 0.888 & -32.60 & -5.74 & 13.1 & 10.7 \\
\hline & 1.65 & 1.82 & 17.00 & 15.91 & 2.75 & 1.40 & 0.794 & -84.26 & -71.20 & 23.1 & 20.5 \\
\hline \multirow[t]{3}{*}{ NGC 6809} & 0.11 & 1.77 & 7.73 & 6.03 & 6.33 & 4.21 & 0.556 & -12.48 & 25.49 & 23.9 & 23.3 \\
\hline & 0.39 & 1.80 & 11.86 & 6.82 & 6.28 & 3.67 & 0.578 & 7.20 & 61.32 & 30.5 & 32.0 \\
\hline & 0.07 & 1.14 & 8.55 & 7.45 & 7.80 & 4.45 & 0.746 & -22.44 & 7.10 & 17.3 & 15.4 \\
\hline \multirow[t]{3}{*}{ NGC 6838} & 4.66 & 4.69 & 8.42 & 7.74 & 0.35 & 0.30 & 0.244 & 121.05 & 131.50 & 26.6 & 30.0 \\
\hline & 4.01 & 4.07 & 9.23 & 8.18 & 0.41 & 0.32 & 0.333 & 109.22 & 126.49 & 24.3 & 26.5 \\
\hline & 5.28 & 5.39 & 7.90 & 7.24 & 0.38 & 0.34 & 0.146 & 129.07 & 137.68 & 29.3 & 33.8 \\
\hline \multirow[t]{3}{*}{ NGC 6934} & 6.91 & 7.21 & 56.21 & 55.73 & 54.84 & 31.27 & 0.771 & -60.20 & -58.98 & 58.7 & 53.9 \\
\hline & 2.25 & 3.14 & 27.40 & 25.79 & 27.12 & 15.56 & 0.783 & -22.81 & -10.83 & 33.5 & 30.3 \\
\hline & 9.95 & 10.08 & 211.67 & 211.41 & 200.73 & 115.01 & 0.909 & -110.31 & -110.19 & 75.3 & 64.2 \\
\hline \multirow[t]{3}{*}{ NGC 7006} & 18.25 & 18.28 & 111.37 & 111.35 & 56.13 & 31.57 & 0.718 & 600.48 & 600.53 & 122.4 & 114.2 \\
\hline & 6.84 & 6.93 & 57.04 & 56.00 & 21.67 & 11.52 & 0.780 & 261.30 & 263.45 & 61.6 & 57.1 \\
\hline & $\ldots$ & $\ldots$ & $\ldots$ & $\ldots$ & $\ldots$ & $\ldots$ & $\ldots$ & $\ldots$ & $\ldots$ & $\ldots$ & $\ldots$ \\
\hline \multirow[t]{3}{*}{ NGC 7078} & 6.12 & 6.40 & 12.15 & 11.96 & 7.73 & 6.13 & 0.303 & 123.83 & 126.38 & 97.5 & 105.1 \\
\hline & 3.97 & 4.32 & 13.14 & 11.66 & 6.75 & 4.50 & 0.458 & 98.30 & 111.44 & 74.5 & 76.4 \\
\hline & 8.76 & 8.97 & 14.93 & 14.72 & 10.46 & 8.48 & 0.243 & 156.46 & 157.23 & 123.9 & 134.6 \\
\hline \multirow[t]{3}{*}{ NGC 7089} & 6.59 & 6.99 & 43.88 & 43.41 & 41.28 & 23.76 & 0.723 & -76.05 & -74.72 & 96.5 & 89.9 \\
\hline & 5.60 & 6.10 & 23.72 & 23.17 & 23.15 & 14.65 & 0.583 & -40.60 & -38.26 & 88.3 & 87.0 \\
\hline & 8.48 & 8.72 & 91.04 & 90.59 & 85.06 & 47.35 & 0.824 & -127.98 & -127.41 & 112.1 & 98.1 \\
\hline NGC 7099 & 3.63 & 3.94 & 7.87 & 7.58 & 6.25 & 4.95 & 0.316 & -58.60 & -55.62 & 42.2 & 45.5 \\
\hline
\end{tabular}


Table 2-Continued

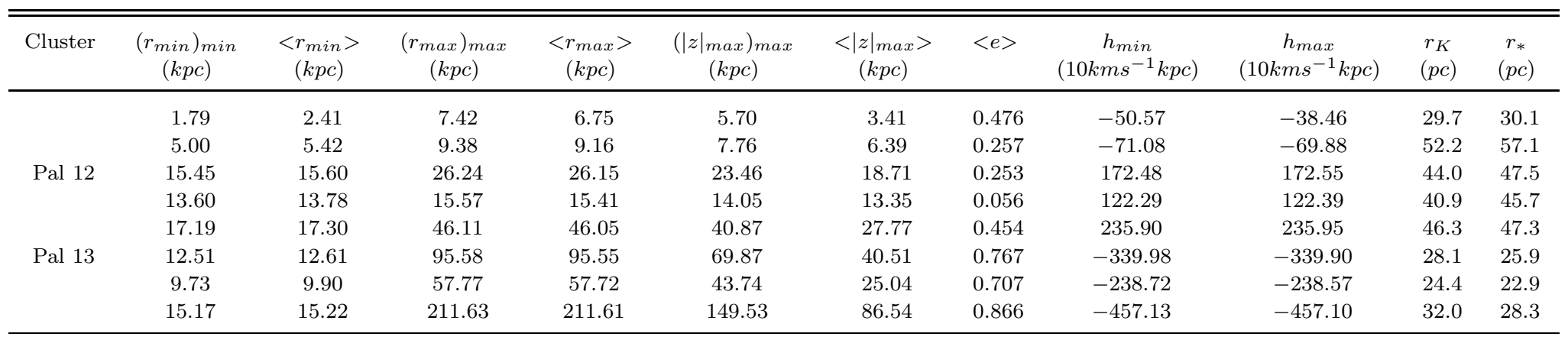


Table 3. Destruction Rates

\begin{tabular}{|c|c|c|c|c|c|c|c|c|c|}
\hline Cluster & $\begin{array}{c}M_{c} \\
\left(M_{\odot}\right)\end{array}$ & $c$ & $\begin{array}{l}r_{h} \\
(p c)\end{array}$ & $\begin{array}{c}<1 / t_{\text {bulge }, 1}> \\
\left(y r^{-1}\right)\end{array}$ & $\begin{array}{c}<1 / t_{\text {bulge }, 2}> \\
\left(y r^{-1}\right)\end{array}$ & $\begin{array}{c}<1 / t_{\text {bulge }}> \\
\quad\left(y r^{-1}\right)\end{array}$ & $\begin{array}{c}<1 / t_{d i s k, 1}> \\
\left(y r^{-1}\right)\end{array}$ & $\begin{array}{c}<1 / t_{d i s k, 2}> \\
\left(y r^{-1}\right)\end{array}$ & $\begin{array}{c}<1 / t_{d i s k}> \\
\left(y r^{-1}\right)\end{array}$ \\
\hline \multirow[t]{2}{*}{ NGC 104} & \multirow[t]{2}{*}{$1.0 \mathrm{E} 06$} & \multirow[t]{2}{*}{2.03} & \multirow[t]{2}{*}{3.65} & $1.1 \mathrm{E}-16$ & $6.4 \mathrm{E}-18$ & $1.1 \mathrm{E}-16$ & $8.8 \mathrm{E}-14$ & $9.8 \mathrm{E}-15$ & $9.8 \mathrm{E}-14$ \\
\hline & & & & $1.3 \mathrm{E}-16$ & $7.5 \mathrm{E}-18$ & $1.3 \mathrm{E}-16$ & 8.7E-14 & $9.6 \mathrm{E}-15$ & $9.7 \mathrm{E}-14$ \\
\hline \multirow[t]{2}{*}{ NGC 288} & \multirow[t]{2}{*}{$8.5 \mathrm{E} 04$} & \multirow[t]{2}{*}{0.96} & \multirow[t]{2}{*}{5.68} & $1.9 \mathrm{E}-13$ & $5.7 \mathrm{E}-14$ & $2.5 \mathrm{E}-13$ & $1.8 \mathrm{E}-12$ & $8.5 \mathrm{E}-13$ & 2.7E-12 \\
\hline & & & & $2.2 \mathrm{E}-13$ & $6.4 \mathrm{E}-14$ & $2.8 \mathrm{E}-13$ & $1.7 \mathrm{E}-12$ & $8.2 \mathrm{E}-13$ & $2.6 \mathrm{E}-12$ \\
\hline \multirow[t]{2}{*}{ NGC 362} & \multirow[t]{2}{*}{$3.9 \mathrm{E} 05$} & \multirow[t]{2}{*}{1.94} & \multirow[t]{2}{*}{2.00} & $4.4 \mathrm{E}-11$ & $6.4 \mathrm{E}-12$ & $5.0 \mathrm{E}-11$ & $7.7 \mathrm{E}-13$ & $8.5 \mathrm{E}-14$ & $8.6 \mathrm{E}-13$ \\
\hline & & & & $8.1 \mathrm{E}-12$ & $1.1 \mathrm{E}-12$ & $9.3 \mathrm{E}-12$ & $4.8 \mathrm{E}-13$ & $5.9 \mathrm{E}-14$ & $5.3 \mathrm{E}-13$ \\
\hline \multirow[t]{2}{*}{ NGC 1851} & \multirow[t]{2}{*}{ 3.7E05 } & \multirow[t]{2}{*}{2.32} & \multirow[t]{2}{*}{1.83} & $1.5 \mathrm{E}-16$ & $7.6 \mathrm{E}-18$ & $1.5 \mathrm{E}-16$ & $6.9 \mathrm{E}-15$ & $5.8 \mathrm{E}-16$ & $7.5 \mathrm{E}-15$ \\
\hline & & & & $1.5 \mathrm{E}-16$ & $7.6 \mathrm{E}-18$ & $1.6 \mathrm{E}-16$ & $6.8 \mathrm{E}-15$ & $5.7 \mathrm{E}-16$ & $7.4 \mathrm{E}-15$ \\
\hline \multirow[t]{2}{*}{ NGC 1904} & \multirow[t]{2}{*}{$2.4 \mathrm{E} 05$} & \multirow[t]{2}{*}{1.72} & \multirow[t]{2}{*}{3.00} & $1.3 \mathrm{E}-15$ & $1.5 \mathrm{E}-16$ & $1.4 \mathrm{E}-15$ & $4.2 \mathrm{E}-14$ & $8.0 \mathrm{E}-15$ & $5.0 \mathrm{E}-14$ \\
\hline & & & & $1.5 \mathrm{E}-15$ & $1.8 \mathrm{E}-16$ & $1.7 \mathrm{E}-15$ & $5.0 \mathrm{E}-14$ & $1.0 \mathrm{E}-14$ & $5.9 \mathrm{E}-14$ \\
\hline \multirow[t]{2}{*}{ NGC 2298} & \multirow[t]{2}{*}{$5.7 \mathrm{E} 04$} & \multirow[t]{2}{*}{1.28} & \multirow[t]{2}{*}{2.43} & $1.8 \mathrm{E}-15$ & $2.6 \mathrm{E}-16$ & $2.0 \mathrm{E}-15$ & $9.1 \mathrm{E}-14$ & $2.5 \mathrm{E}-14$ & $1.2 \mathrm{E}-13$ \\
\hline & & & & $1.8 \mathrm{E}-15$ & $2.6 \mathrm{E}-16$ & $2.0 \mathrm{E}-15$ & $8.8 \mathrm{E}-14$ & $2.4 \mathrm{E}-14$ & $1.1 \mathrm{E}-13$ \\
\hline \multirow[t]{2}{*}{ NGC 4147} & \multirow[t]{2}{*}{ 5.0E04 } & \multirow[t]{2}{*}{1.80} & 2.41 & $9.0 \mathrm{E}-13$ & $1.5 \mathrm{E}-13$ & $1.1 \mathrm{E}-12$ & $4.5 \mathrm{E}-13$ & $9.7 \mathrm{E}-14$ & $5.5 \mathrm{E}-13$ \\
\hline & & & & $9.5 \mathrm{E}-13$ & $1.6 \mathrm{E}-13$ & $1.1 \mathrm{E}-12$ & $5.8 \mathrm{E}-13$ & $1.3 \mathrm{E}-13$ & 7.1E-13 \\
\hline NGC 4590 & $1.5 \mathrm{E} 05$ & 1.64 & 4.60 & $6.3 \mathrm{E}-15$ & $5.8 \mathrm{E}-16$ & $6.9 \mathrm{E}-15$ & $1.1 \mathrm{E}-13$ & $1.5 \mathrm{E}-14$ & $1.2 \mathrm{E}-13$ \\
\hline & & & & $6.4 \mathrm{E}-15$ & $5.9 \mathrm{E}-16$ & $7.0 \mathrm{E}-15$ & $1.1 \mathrm{E}-13$ & $1.5 \mathrm{E}-14$ & $1.2 \mathrm{E}-13$ \\
\hline NGC 5024 & $5.2 \mathrm{E} 05$ & 1.78 & 5.75 & $5.7 \mathrm{E}-17$ & $3.5 \mathrm{E}-18$ & $6.1 \mathrm{E}-17$ & $7.4 \mathrm{E}-16$ & $6.6 \mathrm{E}-17$ & $8.1 \mathrm{E}-16$ \\
\hline & & & & $5.7 \mathrm{E}-17$ & $3.6 \mathrm{E}-18$ & $6.1 \mathrm{E}-17$ & $7.4 \mathrm{E}-16$ & $6.6 \mathrm{E}-17$ & $8.1 \mathrm{E}-16$ \\
\hline NGC 5139 & 3.3E06 & 1.61 & 6.44 & $5.7 \mathrm{E}-12$ & $8.8 \mathrm{E}-13$ & $6.6 \mathrm{E}-12$ & $2.7 \mathrm{E}-12$ & $4.2 \mathrm{E}-13$ & $3.1 \mathrm{E}-12$ \\
\hline & & & & $2.3 \mathrm{E}-12$ & $3.3 \mathrm{E}-13$ & $2.6 \mathrm{E}-12$ & $2.2 \mathrm{E}-12$ & $3.6 \mathrm{E}-13$ & $2.5 \mathrm{E}-12$ \\
\hline NGC 5272 & $6.4 \mathrm{E} 05$ & 1.84 & 3.39 & $1.5 \mathrm{E}-13$ & $9.1 \mathrm{E}-15$ & $1.6 \mathrm{E}-13$ & $9.4 \mathrm{E}-13$ & $8.7 \mathrm{E}-14$ & $1.0 \mathrm{E}-12$ \\
\hline & & & & $1.5 \mathrm{E}-13$ & $9.0 \mathrm{E}-15$ & $1.6 \mathrm{E}-13$ & $8.5 \mathrm{E}-13$ & 8.0E-14 & $9.3 \mathrm{E}-13$ \\
\hline NGC 5466 & $1.0 \mathrm{E} 05$ & 1.32 & 10.41 & $1.1 \mathrm{E}-12$ & $2.1 \mathrm{E}-13$ & $1.3 \mathrm{E}-12$ & $2.4 \mathrm{E}-12$ & $5.9 \mathrm{E}-13$ & 2.9E-12 \\
\hline & & & & $1.1 \mathrm{E}-12$ & $2.2 \mathrm{E}-13$ & $1.3 \mathrm{E}-12$ & $2.4 \mathrm{E}-12$ & $5.8 \mathrm{E}-13$ & $2.9 \mathrm{E}-12$ \\
\hline Pal 5 & $2.0 \mathrm{E} 04$ & 0.70 & 19.97 & $9.3 \mathrm{E}-11$ & $4.9 \mathrm{E}-11$ & $1.4 \mathrm{E}-10$ & $2.6 \mathrm{E}-10$ & $1.5 \mathrm{E}-10$ & 4.1E-10 \\
\hline & & & & $9.3 \mathrm{E}-11$ & $4.9 \mathrm{E}-11$ & $1.4 \mathrm{E}-10$ & $2.5 \mathrm{E}-10$ & $1.5 \mathrm{E}-10$ & $4.1 \mathrm{E}-10$ \\
\hline NGC 5897 & 1.3E05 & 0.79 & 7.61 & $1.7 \mathrm{E}-10$ & $8.6 \mathrm{E}-11$ & $2.6 \mathrm{E}-10$ & $1.5 \mathrm{E}-11$ & $7.6 \mathrm{E}-12$ & $2.3 \mathrm{E}-11$ \\
\hline & & & & $3.6 \mathrm{E}-11$ & $1.6 \mathrm{E}-11$ & $5.2 \mathrm{E}-11$ & $1.5 \mathrm{E}-11$ & $7.5 \mathrm{E}-12$ & 2.3E-11 \\
\hline NGC 5904 & $5.7 \mathrm{E} 05$ & 1.83 & 4.60 & $5.6 \mathrm{E}-13$ & $1.0 \mathrm{E}-13$ & $6.6 \mathrm{E}-13$ & $1.6 \mathrm{E}-13$ & $3.6 \mathrm{E}-14$ & $1.9 \mathrm{E}-13$ \\
\hline & & & & $4.8 \mathrm{E}-13$ & 8.7E-14 & $5.7 \mathrm{E}-13$ & $1.5 \mathrm{E}-13$ & 3.3E-14 & $1.8 \mathrm{E}-13$ \\
\hline NGC 6093 & 3.3E05 & 1.95 & 1.89 & $3.9 \mathrm{E}-14$ & $2.6 \mathrm{E}-15$ & $4.1 \mathrm{E}-14$ & $1.1 \mathrm{E}-12$ & $1.4 \mathrm{E}-13$ & 1.3E-12 \\
\hline & & & & $1.6 \mathrm{E}-13$ & $1.3 \mathrm{E}-14$ & $1.8 \mathrm{E}-13$ & $1.1 \mathrm{E}-12$ & $1.3 \mathrm{E}-13$ & $1.2 \mathrm{E}-12$ \\
\hline NGC 6121 & 1.3E05 & 1.59 & 2.33 & $1.8 \mathrm{E}-10$ & $5.7 \mathrm{E}-11$ & $2.3 \mathrm{E}-10$ & $7.4 \mathrm{E}-13$ & $1.4 \mathrm{E}-13$ & 8.9E-13 \\
\hline & & & & $2.9 \mathrm{E}-11$ & $8.3 \mathrm{E}-12$ & $3.8 \mathrm{E}-11$ & $6.8 \mathrm{E}-13$ & $1.2 \mathrm{E}-13$ & $8.0 \mathrm{E}-13$ \\
\hline
\end{tabular}


Table 3-Continued

\begin{tabular}{|c|c|c|c|c|c|c|c|c|c|}
\hline Cluster & $\begin{array}{c}M_{c} \\
\left(M_{\odot}\right)\end{array}$ & $c$ & $\begin{array}{l}r_{h} \\
(p c)\end{array}$ & $\begin{array}{c}<1 / t_{\text {bulge }, 1}> \\
\left(y r^{-1}\right)\end{array}$ & $\begin{array}{c}<1 / t_{\text {bulge }, 2}> \\
\left(y r^{-1}\right)\end{array}$ & $\begin{array}{c}<1 / t_{\text {bulge }}> \\
\left(y r^{-1}\right)\end{array}$ & $\begin{array}{c}<1 / t_{\text {disk }, 1}> \\
\left(y r^{-1}\right)\end{array}$ & $\begin{array}{c}<1 / t_{\text {disk }, 2}> \\
\quad\left(y r^{-1}\right)\end{array}$ & $\begin{array}{c}<1 / t_{\text {disk }}> \\
\left(y r^{-1}\right)\end{array}$ \\
\hline \multirow[t]{2}{*}{ NGC 6144} & \multirow[t]{2}{*}{ 8.6Е04 } & \multirow[t]{2}{*}{1.55} & \multirow[t]{2}{*}{4.00} & $1.4 \mathrm{E}-10$ & $1.9 \mathrm{E}-11$ & $1.6 \mathrm{E}-10$ & $2.8 \mathrm{E}-10$ & $5.0 \mathrm{E}-11$ & $3.3 \mathrm{E}-10$ \\
\hline & & & & $1.6 \mathrm{E}-10$ & $2.2 \mathrm{E}-11$ & $1.8 \mathrm{E}-10$ & $2.3 \mathrm{E}-10$ & $4.0 \mathrm{E}-11$ & $2.7 \mathrm{E}-10$ \\
\hline \multirow[t]{2}{*}{ NGC 6171} & \multirow[t]{2}{*}{$1.2 \mathrm{E} 05$} & \multirow[t]{2}{*}{1.51} & \multirow[t]{2}{*}{5.03} & $2.4 \mathrm{E}-12$ & $6.6 \mathrm{E}-13$ & $3.0 \mathrm{E}-12$ & $8.9 \mathrm{E}-12$ & $3.4 \mathrm{E}-12$ & $1.2 \mathrm{E}-11$ \\
\hline & & & & $5.7 \mathrm{E}-12$ & $1.8 \mathrm{E}-12$ & $7.5 \mathrm{E}-12$ & $8.4 \mathrm{E}-12$ & $3.2 \mathrm{E}-12$ & $1.2 \mathrm{E}-11$ \\
\hline \multirow[t]{2}{*}{ NGC 6205} & \multirow[t]{2}{*}{$5.2 \mathrm{E} 05$} & \multirow[t]{2}{*}{1.51} & \multirow[t]{2}{*}{3.34} & $5.5 \mathrm{E}-16$ & $4.3 \mathrm{E}-17$ & $5.9 \mathrm{E}-16$ & $2.5 \mathrm{E}-14$ & $3.9 \mathrm{E}-15$ & $2.9 \mathrm{E}-14$ \\
\hline & & & & $5.5 \mathrm{E}-16$ & $4.3 \mathrm{E}-17$ & $5.9 \mathrm{E}-16$ & $2.5 \mathrm{E}-14$ & $3.9 \mathrm{E}-15$ & $2.9 \mathrm{E}-14$ \\
\hline \multirow[t]{2}{*}{ NGC 6218} & \multirow[t]{2}{*}{$1.4 \mathrm{E} 05$} & \multirow[t]{2}{*}{1.39} & \multirow[t]{2}{*}{3.08} & $2.2 \mathrm{E}-13$ & $4.1 \mathrm{E}-14$ & $2.6 \mathrm{E}-13$ & $9.9 \mathrm{E}-13$ & $2.6 \mathrm{E}-13$ & $1.3 \mathrm{E}-12$ \\
\hline & & & & $1.2 \mathrm{E}-13$ & $2.2 \mathrm{E}-14$ & $1.4 \mathrm{E}-13$ & $9.5 \mathrm{E}-13$ & $2.5 \mathrm{E}-13$ & $1.2 \mathrm{E}-12$ \\
\hline \multirow[t]{2}{*}{ NGC 6254} & \multirow[t]{2}{*}{ 1.7E05 } & \multirow[t]{2}{*}{1.40} & \multirow[t]{2}{*}{2.32} & $1.0 \mathrm{E}-14$ & $1.0 \mathrm{E}-15$ & $1.1 \mathrm{E}-14$ & $5.8 \mathrm{E}-13$ & $9.8 \mathrm{E}-14$ & $6.8 \mathrm{E}-13$ \\
\hline & & & & $7.8 \mathrm{E}-15$ & $7.8 \mathrm{E}-16$ & $8.6 \mathrm{E}-15$ & $5.3 \mathrm{E}-13$ & $8.9 \mathrm{E}-14$ & $6.2 \mathrm{E}-13$ \\
\hline \multirow[t]{2}{*}{ NGC 6266} & \multirow[t]{2}{*}{$8.1 \mathrm{E} 05$} & \multirow[t]{2}{*}{1.70} & \multirow[t]{2}{*}{2.47} & $1.6 \mathrm{E}-14$ & $2.0 \mathrm{E}-15$ & $1.8 \mathrm{E}-14$ & $1.1 \mathrm{E}-14$ & $1.5 \mathrm{E}-15$ & $1.2 \mathrm{E}-14$ \\
\hline & & & & $3.3 \mathrm{E}-15$ & $4.0 \mathrm{E}-16$ & $3.7 \mathrm{E}-15$ & $1.0 \mathrm{E}-14$ & $1.4 \mathrm{E}-15$ & $1.1 \mathrm{E}-14$ \\
\hline \multirow[t]{2}{*}{ NGC 6304} & \multirow[t]{2}{*}{$1.5 \mathrm{E} 05$} & \multirow[t]{2}{*}{1.80} & 2.46 & $6.5 \mathrm{E}-13$ & $1.0 \mathrm{E}-13$ & $7.5 \mathrm{E}-13$ & $1.3 \mathrm{E}-12$ & $2.0 \mathrm{E}-13$ & $1.5 \mathrm{E}-12$ \\
\hline & & & & $1.5 \mathrm{E}-13$ & $2.1 \mathrm{E}-14$ & $1.7 \mathrm{E}-13$ & $7.4 \mathrm{E}-13$ & $1.1 \mathrm{E}-13$ & $8.4 \mathrm{E}-13$ \\
\hline NGC 6316 & 3.7E05 & 1.55 & 2.27 & $1.8 \mathrm{E}-12$ & 3.3E-13 & $2.1 \mathrm{E}-12$ & $1.0 \mathrm{E}-13$ & $1.6 \mathrm{E}-14$ & $1.2 \mathrm{E}-13$ \\
\hline & & & & $1.5 \mathrm{E}-12$ & $3.0 \mathrm{E}-13$ & $1.8 \mathrm{E}-12$ & $8.5 \mathrm{E}-14$ & $1.4 \mathrm{E}-14$ & $9.8 \mathrm{E}-14$ \\
\hline NGC 6341 & 3.3E05 & 1.81 & 2.60 & $5.8 \mathrm{E}-12$ & $9.4 \mathrm{E}-13$ & $6.8 \mathrm{E}-12$ & $7.3 \mathrm{E}-13$ & $1.1 \mathrm{E}-13$ & $8.4 \mathrm{E}-13$ \\
\hline & & & & $3.2 \mathrm{E}-12$ & $5.2 \mathrm{E}-13$ & $3.8 \mathrm{E}-12$ & $5.9 \mathrm{E}-13$ & $8.5 \mathrm{E}-14$ & $6.7 \mathrm{E}-13$ \\
\hline NGC 6362 & $1.0 \mathrm{E} 05$ & 1.10 & 4.82 & $6.7 \mathrm{E}-12$ & $1.8 \mathrm{E}-12$ & $8.5 \mathrm{E}-12$ & $2.2 \mathrm{E}-11$ & $6.9 \mathrm{E}-12$ & $2.9 \mathrm{E}-11$ \\
\hline & & & & $4.0 \mathrm{E}-11$ & $1.2 \mathrm{E}-11$ & $5.2 \mathrm{E}-11$ & $3.8 \mathrm{E}-11$ & $1.2 \mathrm{E}-11$ & $5.0 \mathrm{E}-11$ \\
\hline NGC 6397 & 7.7E04 & 2.50 & 1.56 & $3.0 \mathrm{E}-16$ & $3.3 \mathrm{E}-17$ & $3.3 \mathrm{E}-16$ & $1.1 \mathrm{E}-14$ & $1.9 \mathrm{E}-15$ & $1.3 \mathrm{E}-14$ \\
\hline & & & & $2.9 \mathrm{E}-16$ & $3.2 \mathrm{E}-17$ & $3.2 \mathrm{E}-16$ & $1.2 \mathrm{E}-14$ & $2.1 \mathrm{E}-15$ & $1.4 \mathrm{E}-14$ \\
\hline NGC 6522 & $2.0 \mathrm{E} 05$ & 2.50 & 2.36 & $3.1 \mathrm{E}-10$ & $5.2 \mathrm{E}-11$ & $3.6 \mathrm{E}-10$ & $1.4 \mathrm{E}-11$ & $2.2 \mathrm{E}-12$ & $1.6 \mathrm{E}-11$ \\
\hline & & & & $2.1 \mathrm{E}-10$ & $3.9 \mathrm{E}-11$ & $2.5 \mathrm{E}-10$ & $1.1 \mathrm{E}-11$ & $1.7 \mathrm{E}-12$ & $1.3 \mathrm{E}-11$ \\
\hline NGC 6528 & 7.2E04 & 2.29 & 0.99 & $2.2 \mathrm{E}-09$ & $1.8 \mathrm{E}-10$ & $2.4 \mathrm{E}-09$ & $7.8 \mathrm{E}-11$ & $5.0 \mathrm{E}-12$ & $8.3 \mathrm{E}-11$ \\
\hline & & & & $3.4 \mathrm{E}-09$ & $3.2 \mathrm{E}-10$ & 3.7E-09 & $8.8 \mathrm{E}-11$ & $4.6 \mathrm{E}-12$ & $9.3 \mathrm{E}-11$ \\
\hline NGC 6553 & $2.2 \mathrm{E} 05$ & 1.17 & 2.70 & $8.1 \mathrm{E}-16$ & $1.5 \mathrm{E}-16$ & $9.6 \mathrm{E}-16$ & $2.4 \mathrm{E}-15$ & $3.4 \mathrm{E}-16$ & $2.8 \mathrm{E}-15$ \\
\hline & & & & $9.8 \mathrm{E}-16$ & $1.7 \mathrm{E}-16$ & $1.2 \mathrm{E}-15$ & $3.2 \mathrm{E}-15$ & $4.6 \mathrm{E}-16$ & 3.7E-15 \\
\hline NGC 6584 & $2.0 \mathrm{E} 05$ & 1.20 & 3.12 & $4.4 \mathrm{E}-11$ & $1.1 \mathrm{E}-11$ & $5.5 \mathrm{E}-11$ & $1.3 \mathrm{E}-12$ & $2.9 \mathrm{E}-13$ & $1.6 \mathrm{E}-12$ \\
\hline & & & & $7.0 \mathrm{E}-12$ & $1.6 \mathrm{E}-12$ & $8.6 \mathrm{E}-12$ & $1.1 \mathrm{E}-12$ & $2.7 \mathrm{E}-13$ & $1.4 \mathrm{E}-12$ \\
\hline NGC 6626 & $3.2 \mathrm{E} 05$ & 1.67 & 2.54 & $5.1 \mathrm{E}-15$ & $6.4 \mathrm{E}-16$ & $5.7 \mathrm{E}-15$ & $5.0 \mathrm{E}-14$ & $7.1 \mathrm{E}-15$ & $5.8 \mathrm{E}-14$ \\
\hline & & & & $5.3 \mathrm{E}-14$ & $8.6 \mathrm{E}-15$ & $6.1 \mathrm{E}-14$ & $7.8 \mathrm{E}-14$ & $1.2 \mathrm{E}-14$ & $8.9 \mathrm{E}-14$ \\
\hline NGC 6656 & 4.3E05 & 1.31 & 3.03 & $1.9 \mathrm{E}-15$ & $2.3 \mathrm{E}-16$ & $2.1 \mathrm{E}-15$ & $2.8 \mathrm{E}-14$ & $4.6 \mathrm{E}-15$ & $3.3 \mathrm{E}-14$ \\
\hline & & & & $5.4 \mathrm{E}-15$ & $6.8 \mathrm{E}-16$ & $6.1 \mathrm{E}-15$ & $7.1 \mathrm{E}-14$ & $1.3 \mathrm{E}-14$ & $8.4 \mathrm{E}-14$ \\
\hline
\end{tabular}


Table 3-Continued

\begin{tabular}{|c|c|c|c|c|c|c|c|c|c|}
\hline Cluster & $\begin{array}{c}M_{c} \\
\left(M_{\odot}\right)\end{array}$ & $c$ & $\begin{array}{l}r_{h} \\
(p c)\end{array}$ & $\begin{array}{c}<1 / t_{\text {bulge }, 1}> \\
\left(y r^{-1}\right)\end{array}$ & $\begin{array}{c}<1 / t_{\text {bulge }, 2}> \\
\left(y r^{-1}\right)\end{array}$ & $\begin{array}{c}<1 / t_{\text {bulge }}> \\
\left(y r^{-1}\right)\end{array}$ & $\begin{array}{c}<1 / t_{d i s k, 1}> \\
\left(y r^{-1}\right)\end{array}$ & $\begin{array}{c}<1 / t_{\text {disk }, 2}> \\
\quad\left(y r^{-1}\right)\end{array}$ & $\begin{array}{c}<1 / t_{\text {disk }}> \\
\quad\left(y r^{-1}\right)\end{array}$ \\
\hline \multirow[t]{2}{*}{ NGC 6712} & 1.7E05 & 0.90 & 2.75 & $2.5 \mathrm{E}-12$ & $7.8 \mathrm{E}-13$ & $3.3 \mathrm{E}-12$ & $1.3 \mathrm{E}-13$ & $3.5 \mathrm{E}-14$ & $1.6 \mathrm{E}-13$ \\
\hline & & & & $1.3 \mathrm{E}-12$ & $4.1 \mathrm{E}-13$ & $1.7 \mathrm{E}-12$ & $1.1 \mathrm{E}-13$ & $3.1 \mathrm{E}-14$ & $1.5 \mathrm{E}-13$ \\
\hline \multirow[t]{2}{*}{ NGC 6723} & $2.3 \mathrm{E} 05$ & 1.05 & 4.07 & $2.8 \mathrm{E}-13$ & $5.8 \mathrm{E}-14$ & $3.3 \mathrm{E}-13$ & $1.8 \mathrm{E}-12$ & $5.9 \mathrm{E}-13$ & $2.3 \mathrm{E}-12$ \\
\hline & & & & $6.0 \mathrm{E}-13$ & $1.4 \mathrm{E}-13$ & $7.4 \mathrm{E}-13$ & $1.6 \mathrm{E}-12$ & $5.5 \mathrm{E}-13$ & $2.2 \mathrm{E}-12$ \\
\hline \multirow[t]{2}{*}{ NGC 6752} & $2.1 \mathrm{E} 05$ & 2.50 & 2.72 & $3.3 \mathrm{E}-14$ & $2.1 \mathrm{E}-15$ & $3.5 \mathrm{E}-14$ & $1.6 \mathrm{E}-11$ & $1.6 \mathrm{E}-12$ & $1.7 \mathrm{E}-11$ \\
\hline & & & & $1.4 \mathrm{E}-13$ & $1.0 \mathrm{E}-14$ & $1.5 \mathrm{E}-13$ & $1.1 \mathrm{E}-11$ & $1.1 \mathrm{E}-12$ & $1.2 \mathrm{E}-11$ \\
\hline \multirow[t]{2}{*}{ NGC 6779} & $1.5 \mathrm{E} 05$ & 1.37 & 3.41 & $8.0 \mathrm{E}-12$ & $2.5 \mathrm{E}-12$ & $1.1 \mathrm{E}-11$ & $7.0 \mathrm{E}-13$ & $1.5 \mathrm{E}-13$ & $8.5 \mathrm{E}-13$ \\
\hline & & & & $4.0 \mathrm{E}-12$ & $1.2 \mathrm{E}-12$ & $5.2 \mathrm{E}-12$ & $7.1 \mathrm{E}-13$ & $1.5 \mathrm{E}-13$ & $8.6 \mathrm{E}-13$ \\
\hline \multirow[t]{2}{*}{ NGC 6809} & $1.8 \mathrm{E} 05$ & 0.76 & 4.45 & $1.3 \mathrm{E}-11$ & $4.9 \mathrm{E}-12$ & $1.8 \mathrm{E}-11$ & $1.6 \mathrm{E}-12$ & $6.7 \mathrm{E}-13$ & $2.3 \mathrm{E}-12$ \\
\hline & & & & $6.3 \mathrm{E}-12$ & $2.3 \mathrm{E}-12$ & $8.6 \mathrm{E}-12$ & $1.4 \mathrm{E}-12$ & $6.0 \mathrm{E}-13$ & $2.0 \mathrm{E}-12$ \\
\hline \multirow[t]{2}{*}{ NGC 6838} & $3.0 \mathrm{E} 04$ & 1.15 & 1.92 & $8.6 \mathrm{E}-17$ & $1.2 \mathrm{E}-17$ & $9.8 \mathrm{E}-17$ & $1.5 \mathrm{E}-14$ & $1.7 \mathrm{E}-15$ & $1.6 \mathrm{E}-14$ \\
\hline & & & & $9.3 \mathrm{E}-17$ & $1.3 \mathrm{E}-17$ & $1.1 \mathrm{E}-16$ & $1.2 \mathrm{E}-14$ & $1.4 \mathrm{E}-15$ & $1.3 \mathrm{E}-14$ \\
\hline \multirow[t]{2}{*}{ NGC 6934} & $1.6 \mathrm{E} 05$ & 1.53 & 2.74 & $1.4 \mathrm{E}-16$ & $1.3 \mathrm{E}-17$ & $1.6 \mathrm{E}-16$ & $8.1 \mathrm{E}-15$ & $1.5 \mathrm{E}-15$ & $9.6 \mathrm{E}-15$ \\
\hline & & & & $1.4 \mathrm{E}-16$ & $1.3 \mathrm{E}-17$ & $1.6 \mathrm{E}-16$ & $8.1 \mathrm{E}-15$ & $1.5 \mathrm{E}-15$ & $9.6 \mathrm{E}-15$ \\
\hline \multirow[t]{2}{*}{ NGC 7006} & $2.0 \mathrm{E} 05$ & 1.42 & 4.59 & $7.9 \mathrm{E}-18$ & $5.8 \mathrm{E}-19$ & $8.5 \mathrm{E}-18$ & $4.9 \mathrm{E}-17$ & $3.9 \mathrm{E}-18$ & $5.2 \mathrm{E}-17$ \\
\hline & & & & $7.9 \mathrm{E}-18$ & $5.9 \mathrm{E}-19$ & $8.5 \mathrm{E}-18$ & $4.9 \mathrm{E}-17$ & $3.9 \mathrm{E}-18$ & $5.2 \mathrm{E}-17$ \\
\hline \multirow[t]{2}{*}{ NGC 7078} & 8.0E05 & 2.50 & 3.17 & $5.6 \mathrm{E}-16$ & $3.0 \mathrm{E}-17$ & $5.9 \mathrm{E}-16$ & $9.1 \mathrm{E}-14$ & $9.5 \mathrm{E}-15$ & $1.0 \mathrm{E}-13$ \\
\hline & & & & $5.6 \mathrm{E}-16$ & $3.0 \mathrm{E}-17$ & $5.9 \mathrm{E}-16$ & $8.9 \mathrm{E}-14$ & $9.2 \mathrm{E}-15$ & $9.8 \mathrm{E}-14$ \\
\hline \multirow[t]{2}{*}{ NGC 7089} & $6.9 \mathrm{E} 05$ & 1.80 & 3.11 & $3.3 \mathrm{E}-16$ & $2.0 \mathrm{E}-17$ & $3.5 \mathrm{E}-16$ & $1.1 \mathrm{E}-14$ & $1.3 \mathrm{E}-15$ & $1.2 \mathrm{E}-14$ \\
\hline & & & & $3.4 \mathrm{E}-16$ & $2.0 \mathrm{E}-17$ & $3.6 \mathrm{E}-16$ & $1.1 \mathrm{E}-14$ & $1.3 \mathrm{E}-15$ & $1.2 \mathrm{E}-14$ \\
\hline \multirow[t]{2}{*}{ NGC 7099} & $1.6 \mathrm{E} 05$ & 2.50 & 2.68 & $5.4 \mathrm{E}-14$ & $5.0 \mathrm{E}-15$ & $5.9 \mathrm{E}-14$ & $2.1 \mathrm{E}-12$ & $3.4 \mathrm{E}-13$ & $2.4 \mathrm{E}-12$ \\
\hline & & & & $6.1 \mathrm{E}-14$ & $5.7 \mathrm{E}-15$ & $6.7 \mathrm{E}-14$ & $2.0 \mathrm{E}-12$ & $3.3 \mathrm{E}-13$ & $2.4 \mathrm{E}-12$ \\
\hline \multirow[t]{2}{*}{ Pal 12} & 1.1E04 & 1.94 & 7.11 & $1.1 \mathrm{E}-13$ & $1.9 \mathrm{E}-14$ & $1.3 \mathrm{E}-13$ & $1.1 \mathrm{E}-12$ & $2.2 \mathrm{E}-13$ & $1.3 \mathrm{E}-12$ \\
\hline & & & & $1.1 \mathrm{E}-13$ & $1.9 \mathrm{E}-14$ & $1.3 \mathrm{E}-13$ & $1.1 \mathrm{E}-12$ & $2.2 \mathrm{E}-13$ & $1.3 \mathrm{E}-12$ \\
\hline \multirow[t]{2}{*}{ Pal 13} & $5.4 \mathrm{E} 03$ & 0.68 & 3.45 & $9.5 \mathrm{E}-16$ & $2.2 \mathrm{E}-16$ & $1.2 \mathrm{E}-15$ & $1.1 \mathrm{E}-14$ & $3.6 \mathrm{E}-15$ & $1.5 \mathrm{E}-14$ \\
\hline & & & & $9.5 \mathrm{E}-16$ & $2.2 \mathrm{E}-16$ & $1.2 \mathrm{E}-15$ & $1.1 \mathrm{E}-14$ & $3.6 \mathrm{E}-15$ & $1.5 \mathrm{E}-14$ \\
\hline
\end{tabular}



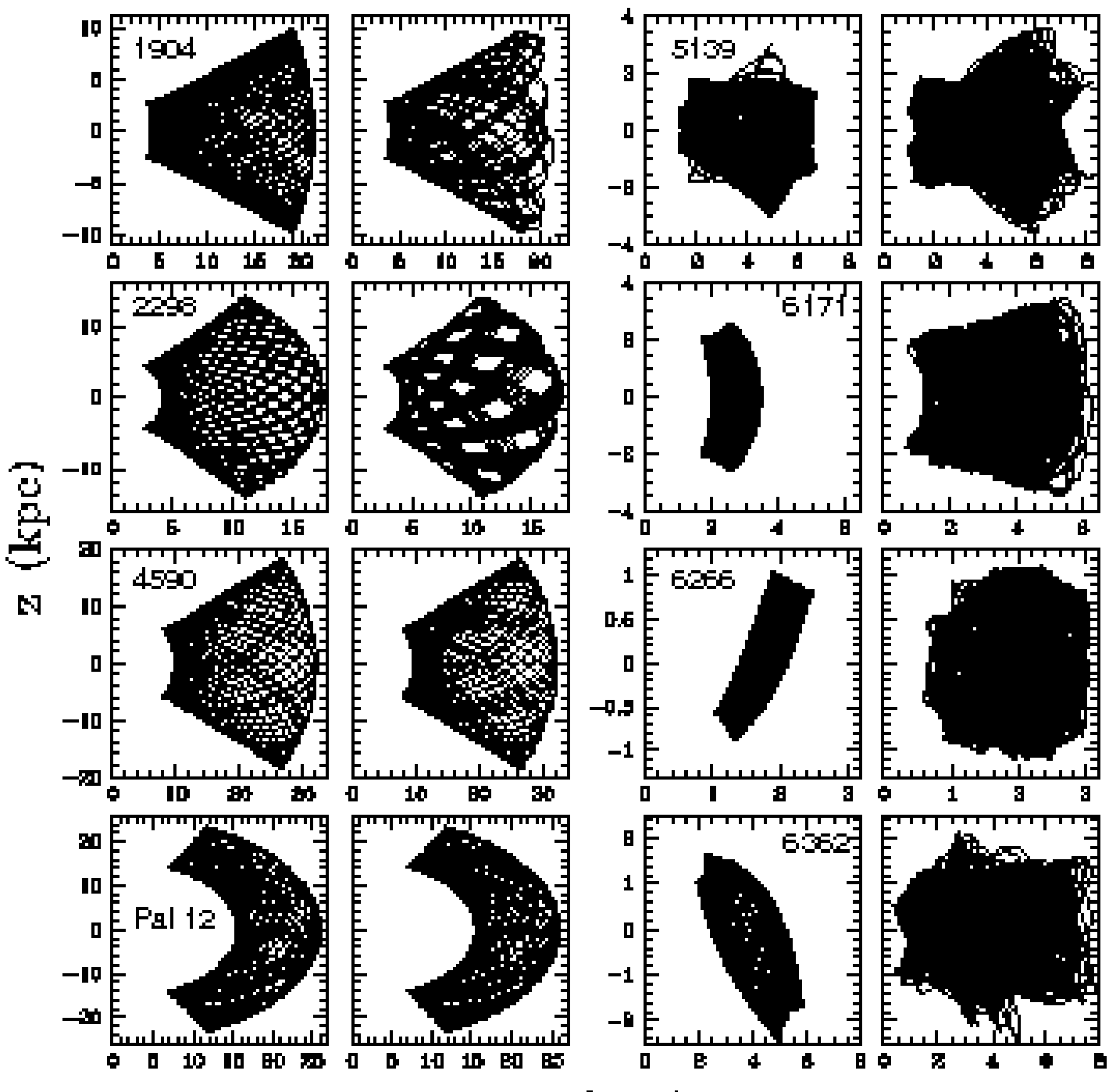

$R(k p e)$

Fig. 1.- Meridional galactic orbits of some clusters. In each pair of frames, the orbits with the axisymmetric and non-axisymmetric potentials are shown on the left and right, respectively. The NGC or Pal number is written in the left frame. In general the scales are not the same in the horizontal and vertical axes. 

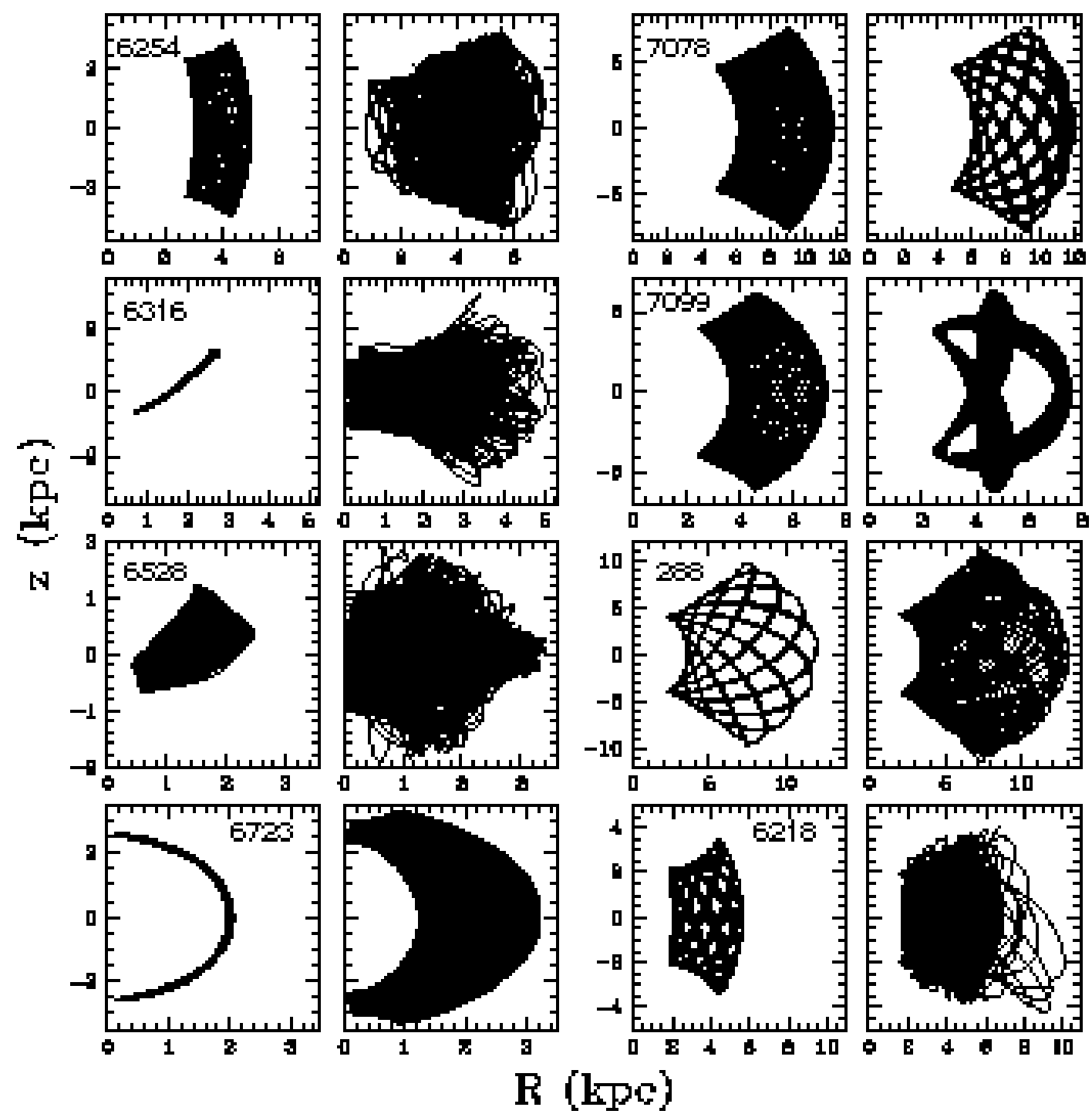

R (kpo)

Fig. 2.- As in Figure 1. 


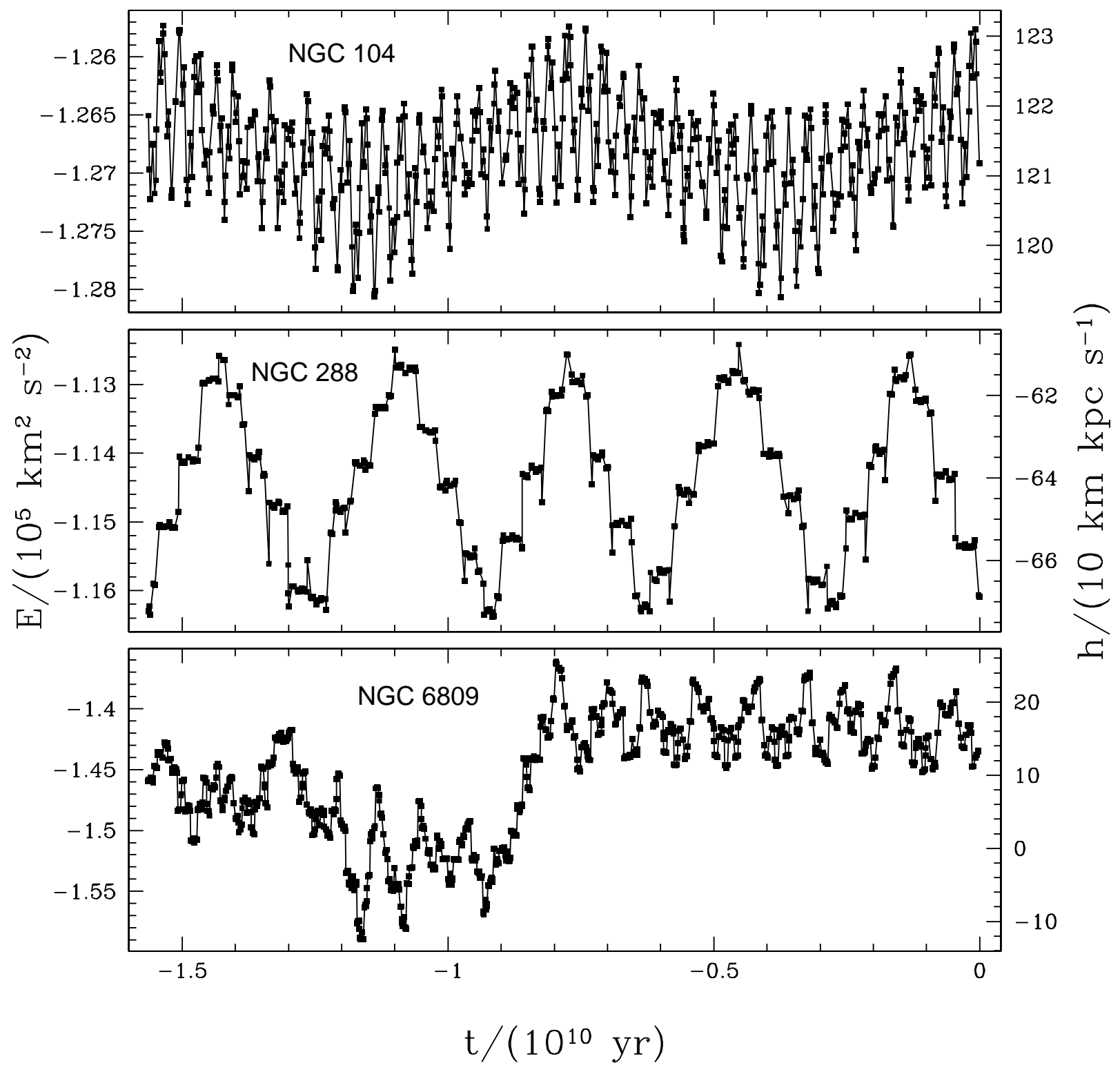

Fig. 3.- Energy and z-angular momentum as functions of time in NGC 104, NGC 288, and NGC 6809 , with the non-axisymmetric potential. $E$ and $h$ are connected by Jacobi's constant $J: J=E-\Omega h ; \Omega$ is the angular velocity of the bar and $h$ is positive in the sense of galactic rotation. 

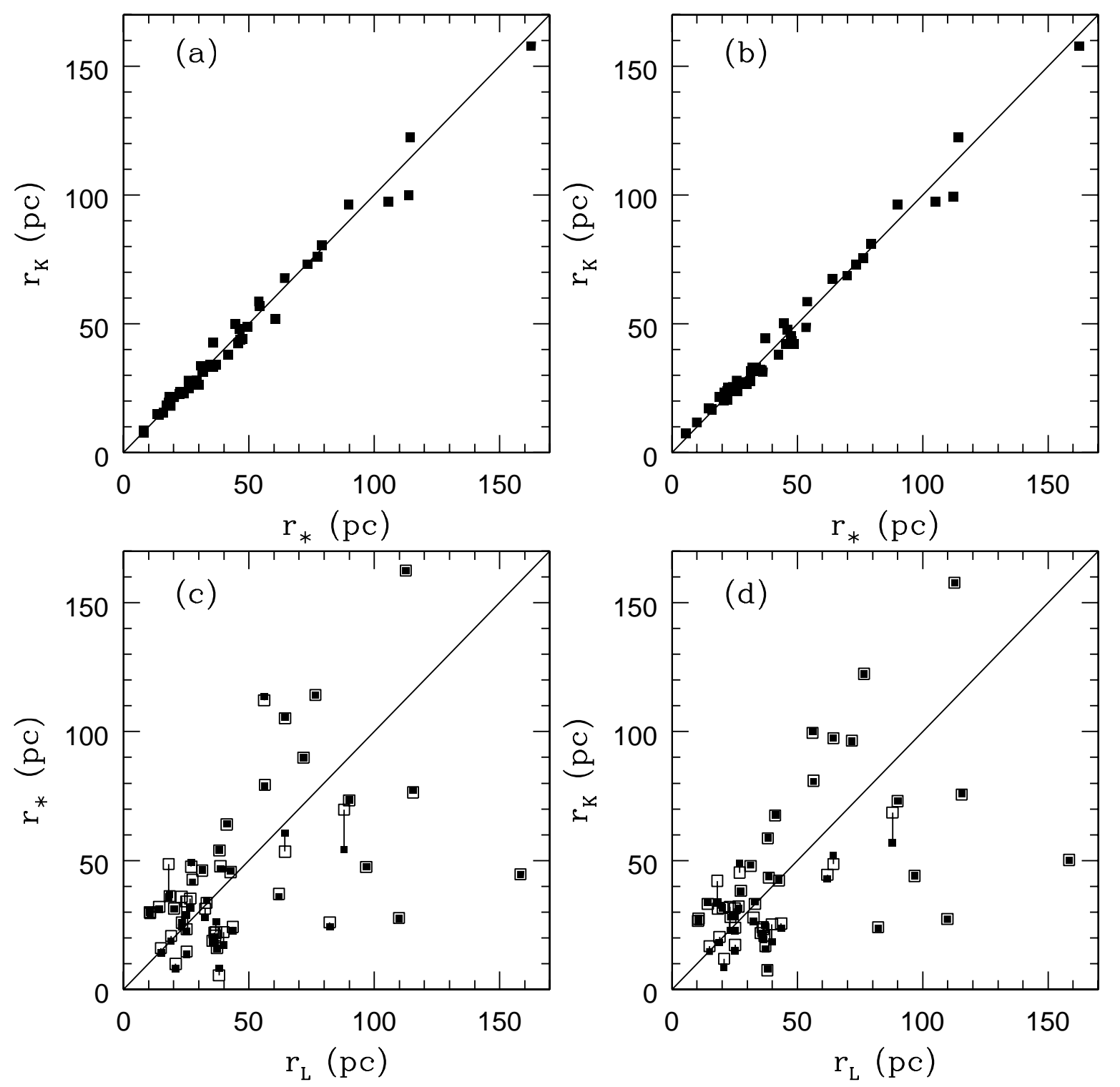

Fig. 4. - Comparison of tidal radii. The tidal radii $r_{K}$ and $r_{*}$ are compared using (a) the axisymmetric, and (b) the non-axisymmetric galactic potentials. In (c) and (d), $r_{*}$ and $r_{K}$ are compared with the observed limiting radius $r_{L}$; filled and empty squares are obtained with the axisymmetric and non-axisymmetric potentials, respectively. In each frame we plot the line of coincidence. 


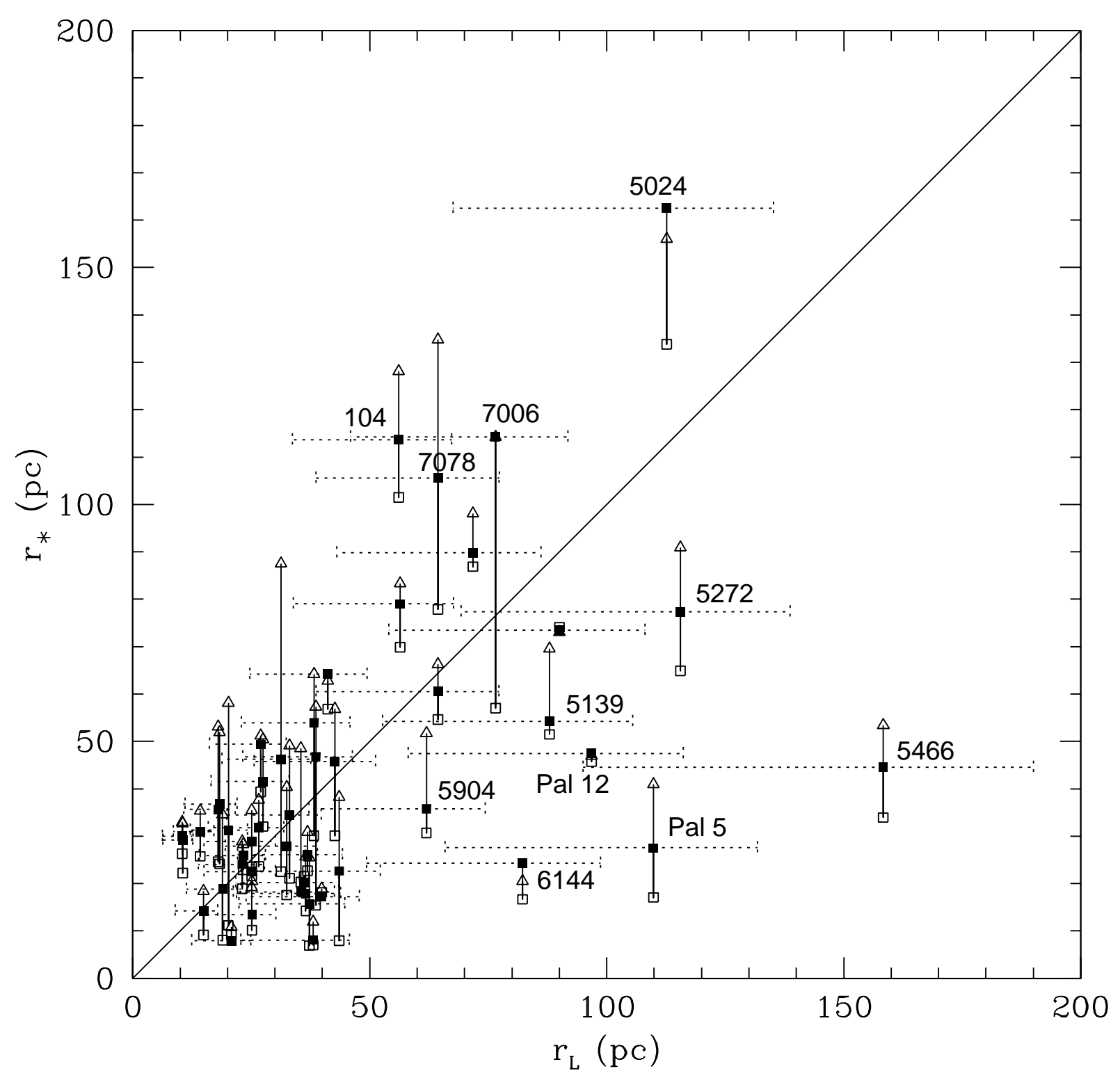

Fig. 5.- Comparison of the tidal radii $r_{*}$ and $r_{L}$ using the axisymmetric potential. For each cluster we plot three points (filled squares, empty squares and empty triangles) corresponding respectively to three orbits: the 'central' orbit and the two extreme orbits of minimum and maximum energy. They appear joined by vertical lines, as 'error bars'. The horizontal dotted lines give the estimated observational uncertainties in $r_{L}$. Some NGC and Pal numbers are shown. The line of coincidence is plotted. 


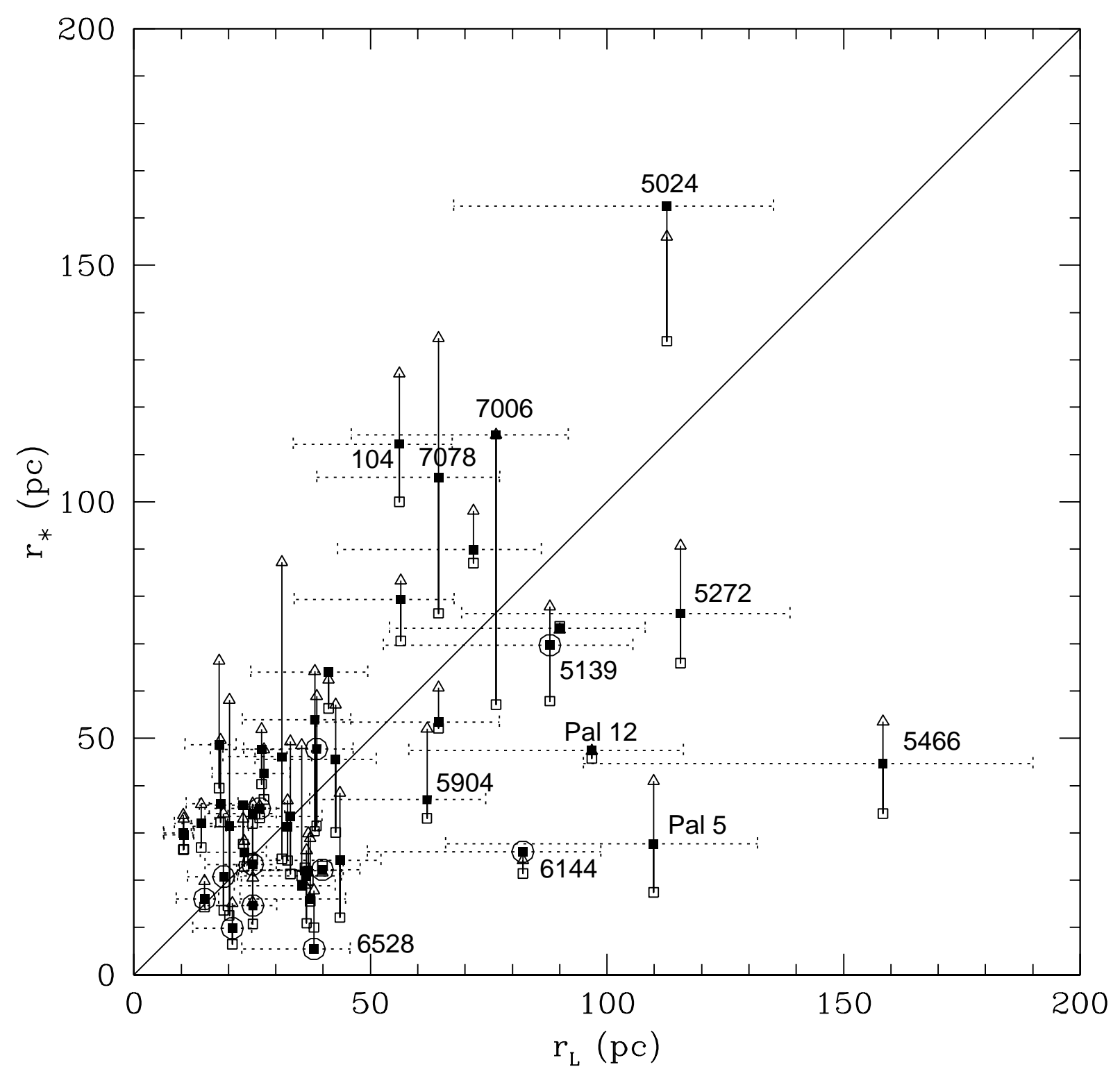

Fig. 6.- As in Figure 5, but now using the non-axisymmetric potential. The central, minimum, and maximum energy orbits are computed with the initial $(t=0)$ conditions of the corresponding orbits in the axisymmetric potential. The filled squares with a circle correspond to clusters with a retrograde galactic orbit, or with an orbit which is both prograde and retrograde, and with a mean perigalactic distance less than $3 \mathrm{kpc}$. 

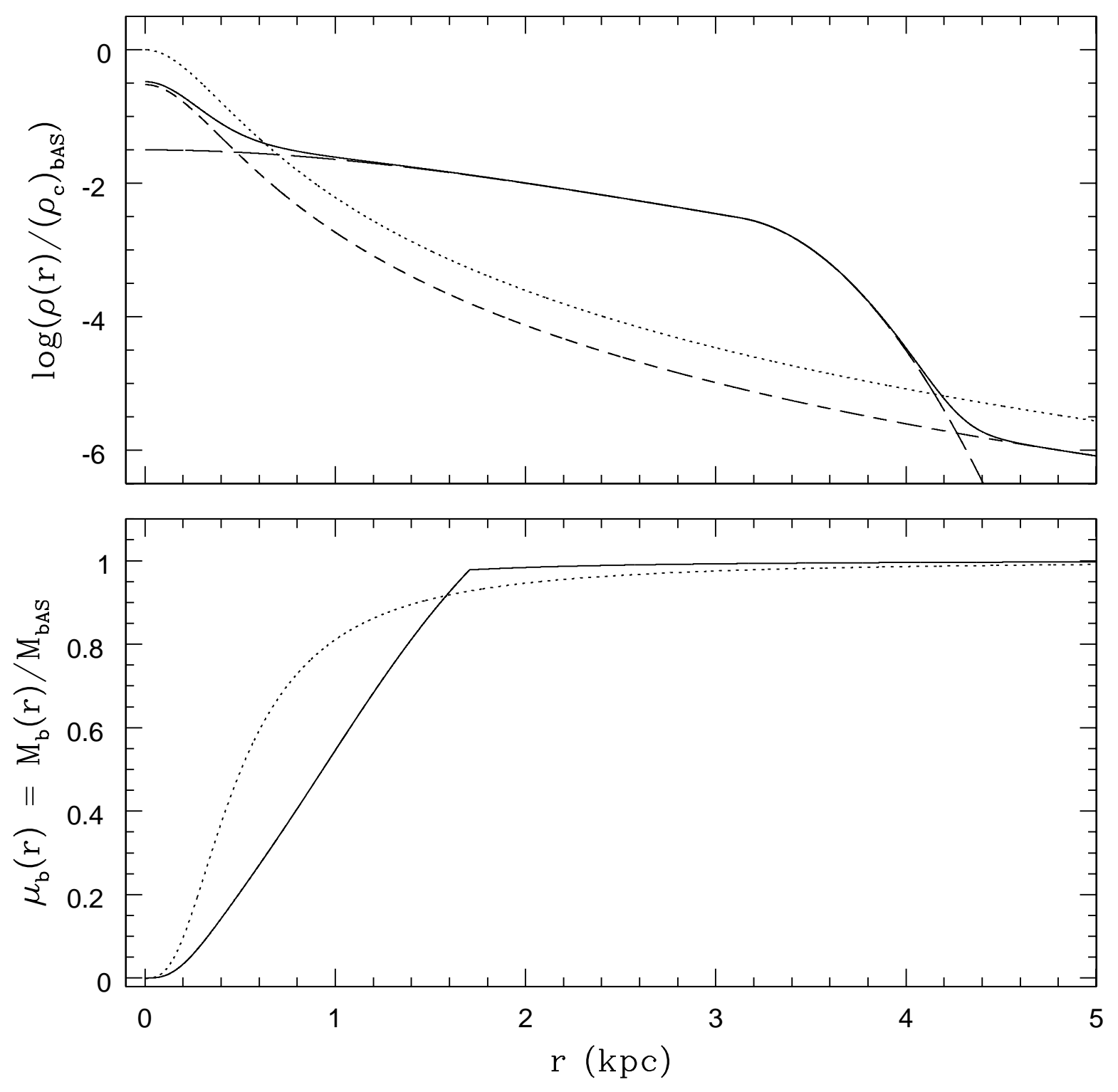

Fig. 7.- The 'bulge' in the non-axisymmetric galactic potential. The upper frame shows the density of the bar and the spherical bulge, scaled by the central density of the bulge in the axisymmetric potential, as functions of galactocentric distance. The dotted line shows the density of the bulge in the axisymmetric galactic potential; the short-dashed line shows the density of the spherical bulge, and the long-dashed line the density of the galactic bar along its major axis. The continuous line gives the total density of both components. For the approximate computation in the non-axisymmetric potential of $\chi\left(\left|\boldsymbol{r}_{p}\right|\right)$ and $\lambda\left(\left|\boldsymbol{r}_{p}\right|,\left|\boldsymbol{r}_{a}\right|\right)$ in Eqs. (3) and (7), in the lower frame the continuous line gives the mass within a sphere of galactocentric radius $r$, scaled by the total mass of the bulge in the axisymmetric galactic potential, of the 'equivalent' total bulge (see the text). The dotted line in this frame corresponds to the bulge in the axisymmetric potential. 


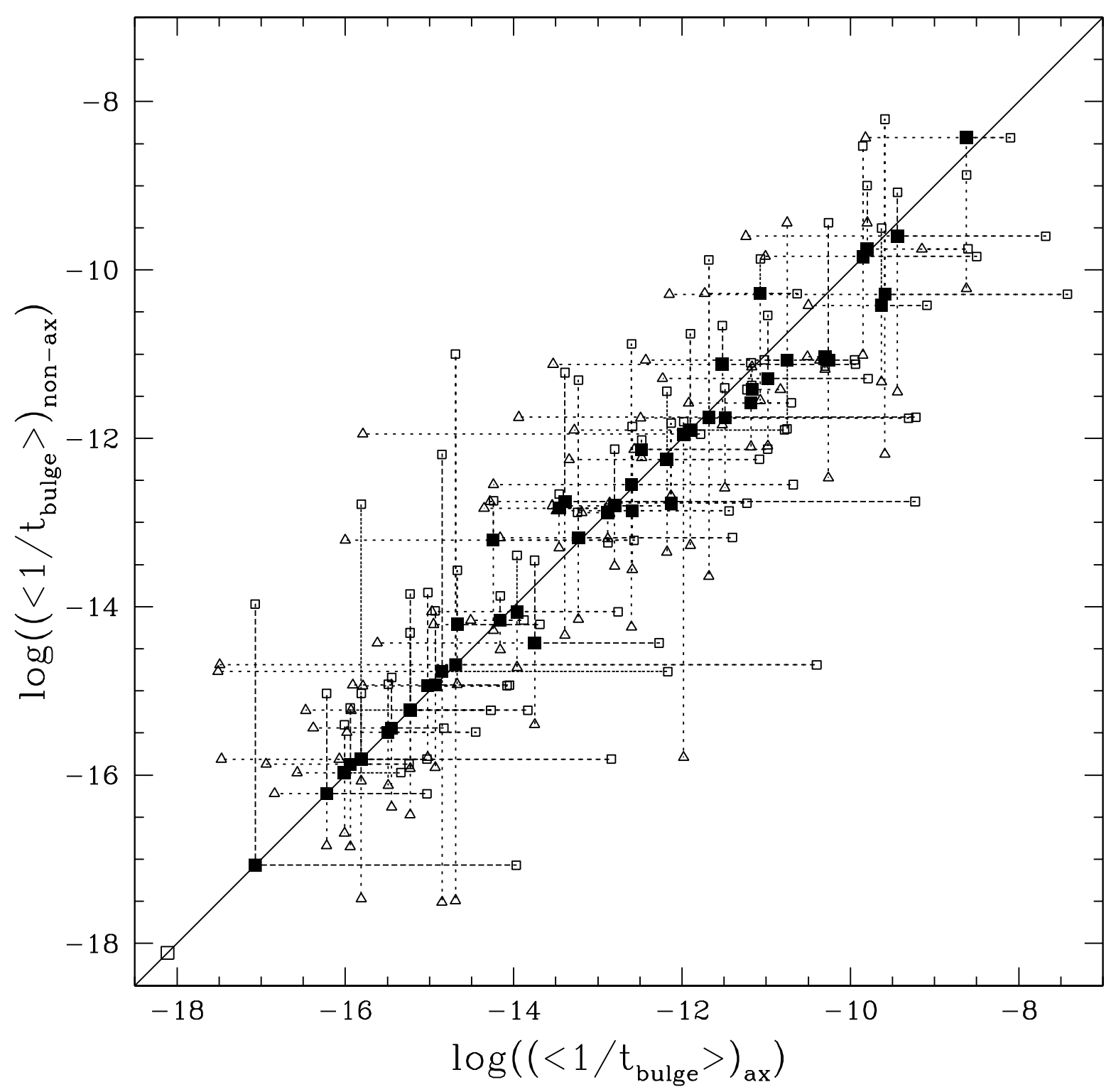

Fig. 8.- Comparison of the destruction rates due to the bulge, in the axisymmetric and nonaxisymmetric potentials. Values obtained with the 'central', minimum energy, and maximum energy orbits are shown as filled squares, empty squares, and empty triangles, respectively. The bottom left empty square corresponds to Pal 3, whose only bound orbit is that of minimum energy. The line of coincidence is plotted. 


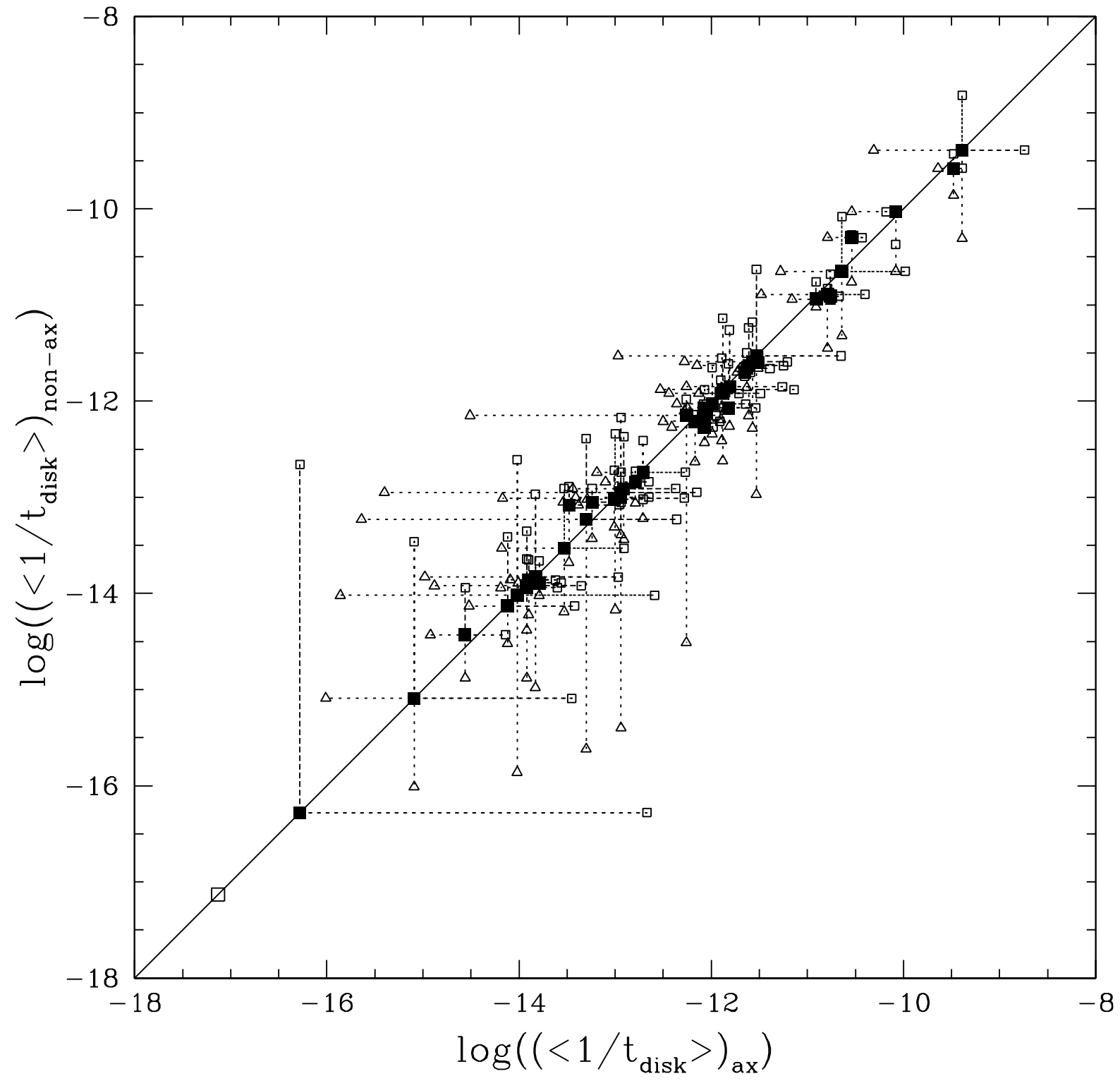

Fig. 9.- Comparison of the destruction rates due to the disk, in the axisymmetric and non-axisymmetric potentials. Points as in Figure 8. 


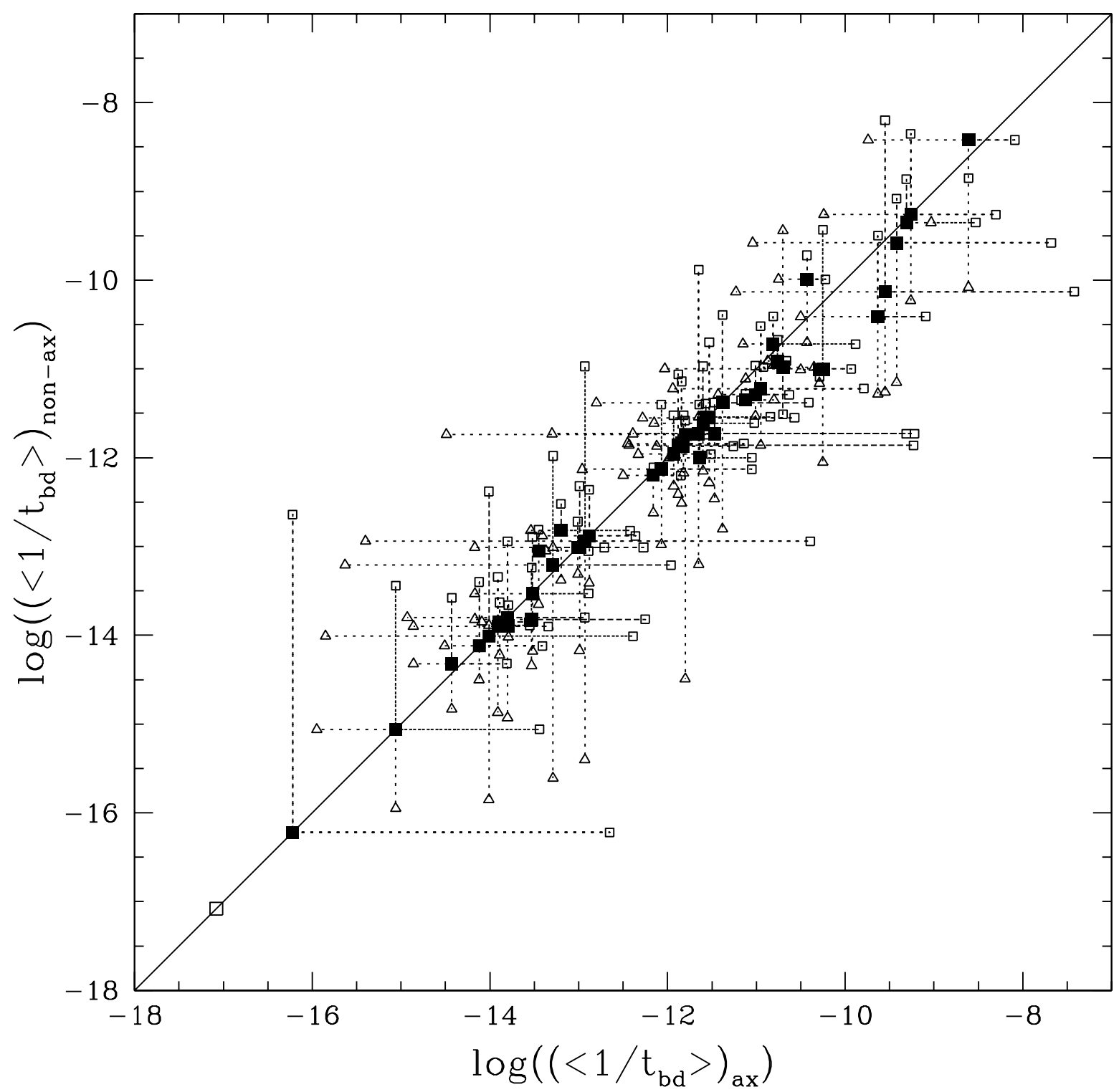

Fig. 10.- Comparison of the total destruction rates due to the bulge and disk, in the axisymmetric and non-axisymmetric potentials. Points as in Figure 8. 


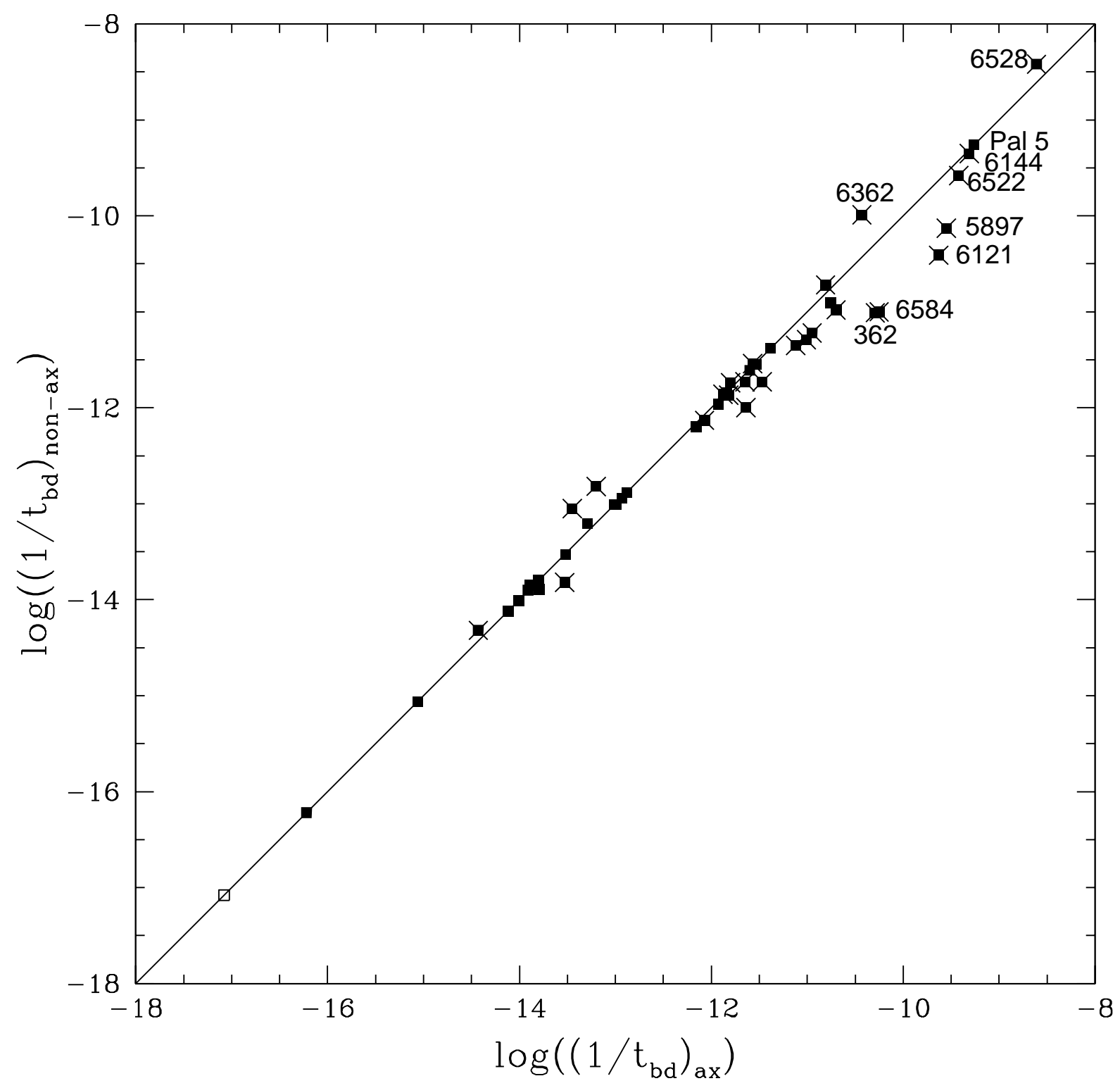

Fig. 11. - Comparison of the total destruction rates due to the bulge and disk, in the axisymmetric and non-axisymmetric potentials. We plot only the points corresponding to the 'central' orbits in Figure 10. Some NGC and Pal numbers are shown. Marked squares correspond to clusters with $<r_{\min }><3 \mathrm{kpc}$. 

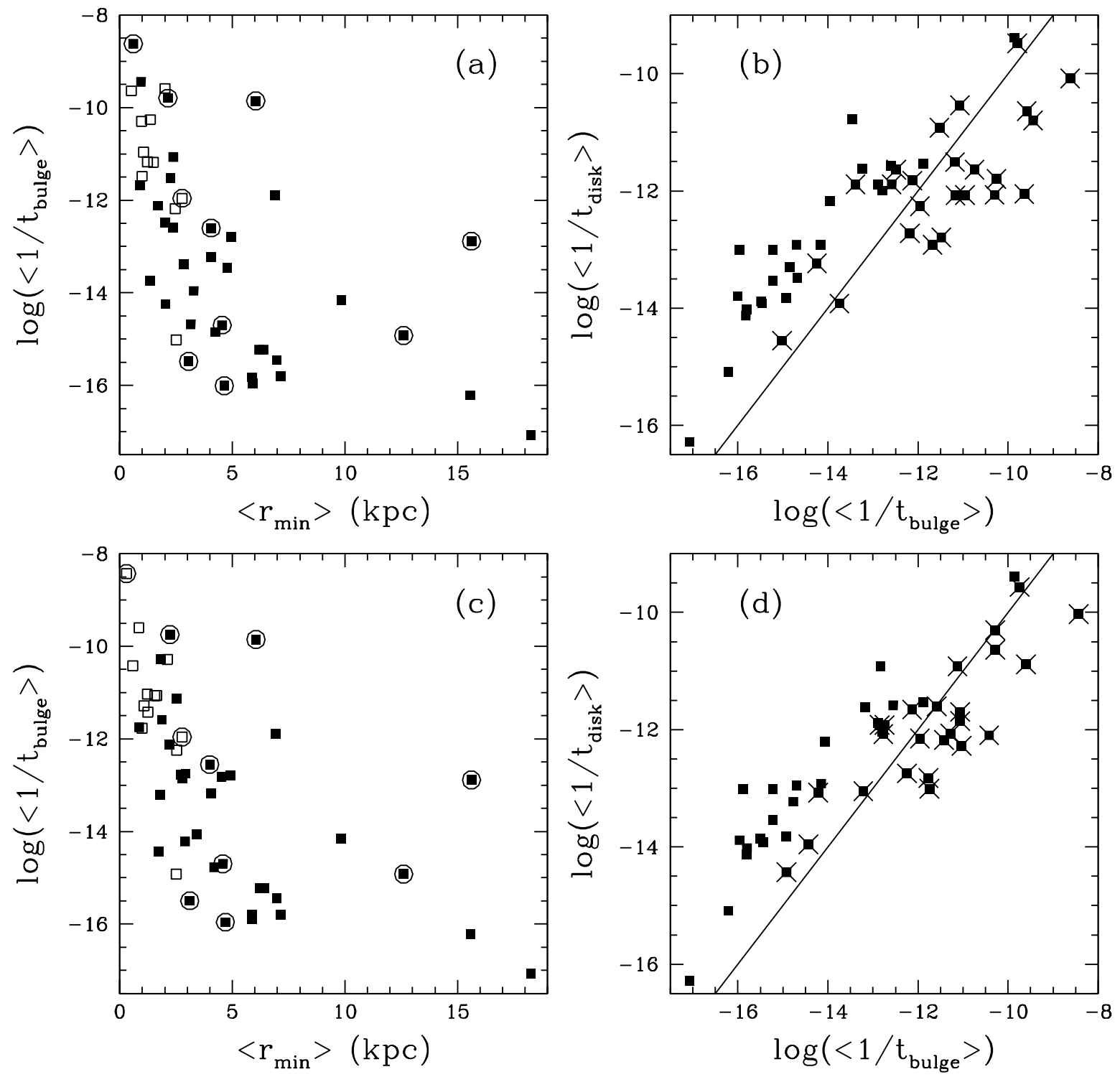

Fig. 12.- Destruction rates with the 'central' orbits. We show the values of the total destruction rate due to the bulge as a function of $\left\langle r_{\min }\right\rangle$, the average minimum distance to the Galactic center, in (a) the axisymmetric, and (c) the non-axisymmetric potential. The squares with a circle correspond to clusters with a mass less than $10^{5} M_{\odot}$, and the empty squares correspond to clusters with $<r_{\min }><3 \mathrm{kpc}$ and orbital eccentricity $e>0.6$. In (b) and (d) we give the comparison of the destruction rates due to the bulge and disk, in the axisymmetric and non-axisymmetric potentials, respectively. Marked squares correspond to clusters with $<r_{\min }><3 \mathrm{kpc}$; the line of coincidence is plotted in both frames. 